\title{
Cellular reprogramming of human monocytes is regulated by time- dependent IL4 signalling and NCOR2
}

Jil Sander ${ }^{*}$, Susanne V. Schmidt ${ }^{2 *}$, Branko Cirovic ${ }^{3}$, Naomi McGovern ${ }^{4,5}$, Olympia Papantonopoulou $^{1}$, Anna-Lena Hardt ${ }^{1}$, Anna C. Aschenbrenner ${ }^{1}$, Christoph Kreer ${ }^{6}$, Thomas Quast $^{7}$, Alexander M. Xu ${ }^{8}$, Lisa M. Schmidleithner ${ }^{1}$, Heidi Theis ${ }^{1}$, Thi Huong Lan Do ${ }^{1}$, Hermi Rizal Bin Sumatoh ${ }^{4}$, Mario A. R. Lauterbach ${ }^{2}$, Jonas Schulte-Schrepping ${ }^{1}$, Patrick Günther ${ }^{1}$, Jia Xue ${ }^{1}$, Kevin Baßler ${ }^{1}$, Thomas Ulas ${ }^{1}$, Kathrin Klee ${ }^{1}$, Stefanie Herresthal ${ }^{1}$, Wolfgang Krebs ${ }^{1}$, Bianca Martin ${ }^{2}$, Eicke Latz ${ }^{2,9,10}$, Kristian Händler ${ }^{1}$, Michael Kraut ${ }^{1}$, Waldemar Kolanus ${ }^{7}$, Marc Beyer ${ }^{1,11}$, Christine S. Falk ${ }^{12}$, Bettina Wiegmann ${ }^{13}$, Sven Burgdorf ${ }^{6}$, Nicholas A. Melosh ${ }^{8}$, Evan W. Newell ${ }^{4}$, Florent Ginhoux ${ }^{4}$, Andreas Schlitzer ${ }^{3,4,15^{*}}$, Joachim L. Schultze ${ }^{1,14^{*}}$

\section{Author Affiliations}

1 Genomics and Immunoregulation, LIMES-Institute, University of Bonn, 53115 Bonn, Germany

2 Institute of Innate Immunity, University Hospital Bonn, University of Bonn, SigmundFreud-Strasse 25, 53127 Bonn, Germany

3 Myeloid Cell Biology, LIMES-Institute, University of Bonn, 53115 Bonn, Germany

4 Agency for Science, Technology and Research (A*STAR), Singapore Immunology Network (SIgN), Singapore

5 Department of Pathology and Center for Trophoblast Research, University of Cambridge, Cambridge, UK

6 Cellular Immunology, LIMES-Institute, University of Bonn, 53115 Bonn, Germany

7 Molecular Immunology \& Cell Biology, LIMES-Institute, University of Bonn, 53115 Bonn, Germany

8 Department of Materials Science and Engineering, Stanford University, Stanford, California 94305, USA

9 Department of Medicine, University of Massachusetts Medical School, Worcester, Massachusetts, USA

${ }^{10}$ German Center for Neurodegenerative Diseases, Bonn, Germany

11 Molecular Immunology, German Center for Neurodegenerative Diseases (DZNE), Sigmund-Freud-Str. 27, 53127 Bonn, Germany

12 Institute of Transplant Immunology, Integrated Research and Treatment Center Transplantation, Hannover Medical School, 30625 Hannover, Germany

13 Department of Cardiothoracic, Transplantation and Vascular Surgery, Hannover Medical School, 30625 Hannover, Germany

14 Platform for Single Cell Genomics and Epigenomics (PRECISE) at the German Center for Neurodegenerative Diseases and the University of Bonn

${ }^{15}$ Lead Contact 


\section{*Authors contributed equally}

Keywords: IL4, human, monocytes, monocyte-derived dendritic cells, macrophages, IL4 activated macrophages (M(IL4)), activation, NCOR2 


\begin{abstract}
The clinical and therapeutic value of human in vitro generated monocyte-derived dendritic cell (moDC) and macrophages is well established. However, in line with recent findings regarding myeloid cell ontogeny and due to our limited understanding of their physiological counterparts, transcriptional regulation and heterogeneity, the full potential of these important cellular systems is still underestimated.

In this study, we use cutting edge high-dimensional analysis methods to better understand the transcriptional organization, phenotypic heterogeneity and functional differences between human ex vivo isolated and in vitro generated mononuclear phagocytes with the aim to better realize their full potential in the clinic.

We demonstrate that human monocytes activated by MCSF or GMCSF most closely resemble inflammatory macrophages identified in vivo, while IL4 signalling in the presence of GMCSF generates moDCs resembling inflammatory DCs in vivo, but not steady state cDC1 or cDC2. Moreover, these reprogramming regimes lead to activated monocytes that present with profoundly different transcriptomic, metabolic, phenotypic and functional profiles. Furthermore, we demonstrate that $\mathrm{CD} 14^{+}$monocytes are integrating multiple exogenous activation signals such as GMCSF and IL4 in a combinatorial and temporal fashion, resulting in a high-dimensional cellular continuum of reprogrammed monocytes dependent on the mode and timing of cytokine exposure. Utilizing nanostraw-based knockdown technology, we demonstrate that the IL4-dependent generation of moDCs relies on the induction, nuclear localization and function of the transcriptional regulator NCOR2.

Finally, we unravel unappreciated heterogeneity within the clinically moDCs population and propose a novel high-dimensional phenotyping strategy to better tailor clinical quality control strategies for patient need and culture conditions to enhance therapeutic outcome.
\end{abstract}




\section{Introduction}

Recently, several transcriptomic, epigenetic, functional and fate-mapping studies established the identity of three different mononuclear cell lineages within the myeloid cell network, macrophages, dendritic cells (DC) and monocytes (Schlitzer et al., 2015a). During murine embryogenesis, different waves of progenitors colonize the developing tissues and establish the initial wave of tissue-resident macrophages, which are long lived and self-maintained in most tissues (Ginhoux and Guilliams, 2016; Ginhoux and Jung, 2014). Human and mouse DC can be separated into three distinct lineages, plasmacytoid DC, conventional DC (cDC) 1 and cDC2 (Merad et al., 2013; Satpathy et al., 2012; Schlitzer and Ginhoux, 2014). Both human and murine $\mathrm{cDC} 1$ and $\mathrm{cDC} 2$ have been shown to arise from specialized precursors within the bone marrow forming their functional specialization early during development, in contrast to the more plastic nature of macrophages (Breton et al., 2015; Ginhoux and Guilliams, 2016; Grajales-Reyes et al., 2015; Heidkamp et al., 2016; Lee et al., 2015; Perdiguero and Geissmann, 2016; Schlitzer et al., 2015b).

The third major component of the mouse and human mononuclear phagocyte network are monocytes. In the mouse, two phenotypically distinct subsets can be delineated, Ly $6 \mathrm{c}^{\text {lo }}$ and Ly6 $\mathrm{c}^{\text {high }}$ monocytes (Schlitzer et al., 2015a; Varol et al., 2015). In human peripheral blood, three different monocyte subsets can be identified by the expression of CD14 and CD16, in which $\mathrm{CD} 14^{+} \mathrm{CD} 16^{-}$monocytes correspond to murine Ly6 $\mathrm{c}^{\text {high }}$ monocytes and CD14-CD16 ${ }^{+}$ monocytes are the counterpart of murine Ly $6 \mathrm{c}^{\text {low }}$ monocytes (Auffray et al., 2009). Yet, little is known, whether this classification relates to functional specialization of distinct functional subsets.

Many studies suggest significant functional overlap between DCs, monocytes and tissue macrophages (Guilliams et al., 2014), including basic mechanisms such as phagocytosis (Fraser et al., 2009), anti-bacterial activity (Fehlings et al., 2012), antigen uptake, processing, and presentation (Cella et al., 1997; Dinter et al., 2014; Randolph et al., 2008), the capacity to activate adaptive immune cells (Toujas et al., 1997), cellular motility (Rossi et al., 2011), and activation programs (Frucht et al., 2000; Krutzik et al., 2005). The nature of this overlap has proven difficult to parse, but new knowledge concerning the distinct ontogeny of these cells has provided the opportunity to re-evaluate and elucidate the division of labor between DCs, monocytes and tissue macrophages.

Currently, assigning specific functions to each cellular entity within the mononuclear phagocyte system requires the use of in vitro systems to simplify complex cellular interactions and instead focus on basic molecular aspects. Murine bone marrow-derived DCs and macrophages cultures driven by granulocyte macrophage colony stimulating factor (GMCSF) or macrophage colony stimulating factor (MCSF) respectively, are frequently used to elucidate and assign molecular mechanisms of classical functions to subsets of mononuclear phagocytes. However these in vitro cultures have been shown to create a heterogeneous set of cells, making attribution of distinct cellular functions difficult (Helft et al., 2015). This conundrum highlights the need for a more detailed investigation of the cellular identity and 
the regulation therof in such in vitro cultures (Guilliams and van de Laar, 2015; Murray et al., 2014; Xue et al., 2014).

Sallusto et al. described the in vitro generation of human monocyte-derived DCs (moDCs) by culturing peripheral blood monocytes with GMCSF and IL4 (Sallusto and Lanzavecchia, 1994). Here, the term moDC was attributed to an activated monocyte population with DC-like functionality based on morphological and functional criteria. Similar phenotypes of functional convergence are observed in in vitro systems of human monocyte-derived macrophages driven by MCSF (moMФ) (Akagawa et al., 2006) or GMCSF (Xue et al., 2014). Furthermore, systems biology-based definitions of mononuclear phagocyte function and nomenclature were established yielding insights about the identity, regulation and developmental origin of those cells (Guilliams and van de Laar, 2015; Murray et al., 2014; Xue et al., 2014). However, studies directly addressing their relationships to mononuclear phagocytes observed in vivo remain limited (Ohradanova-Repic et al., 2016).

Clarifying and assigning true functionality of in vitro generated monocyte-derived phagocytes to their in vivo counterparts and linking this knowledge to our most recent understanding of ontogeny is crucial considering the enormous interest in using in vitro generated mononuclear phagocyte derivatives for immunotherapeutic strategies in the clinic (https://clinicaltrials.gov/ct2/home). Therefore, the functional convergence, plasticity, and heterogeneity of monocyte-derived phagocytes highlighted above paired with the enormous clinical interest raises several important questions. Which part of the mononuclear cell compartment found in vivo do these different human in vitro culture systems represent? What is the difference between moMФ further activated by IL4 (formerly described as 'M2' or better M(IL4) macrophages) and moDCs, which are similarly exposed to IL4, but with different time kinetics? Is there a temporal component in monocyte activation and how is this component integrated on the molecular level? And lastly, how heterogeneous are human in vitro monocyte cultures?

By applying transcriptome analysis combined with bioinformatics, the assessment of cellular phenotype and function, as well as nanostraw-delivered loss of function experiments, we elucidate the relationship of the widely used human moDCs and moMФ systems to cells derived from the mononuclear phagocyte compartment in vivo. We also show that the cellular reprogramming of monocytes in these in vitro culture systems is multifaceted, integrating several time-dependent signals delivered by different growth factors and cytokines such as GMCSF and IL4. Additionally, we show that the time-dependent integration of IL4 during moDC differentiation is orchestrated by the nuclear receptor corepressor 2 (NCOR2). Finally, using mass cytometry, we show that clinically used moDCs are heterogeneous and consist of several different cellular entities. 


\section{Results}

\section{Cellular relationships of in vitro activated human monocyte-derived cells and in vivo mononuclear phagocytes}

Human monocytes activated with MCSF have been used as models for human macrophages (Akagawa et al., 2006), while monocytes activated with GMCSF and IL4 were introduced as models for human DCs (Sallusto and Lanzavecchia, 1994). For clarity and in light of recent findings concerning DC, monocyte, and macrophage ontogeny (Ginhoux and Jung, 2014; Guilliams and van de Laar, 2015; Schlitzer et al., 2015a), we term activated monocytes according to their activation, e.g. monocytes activated with MCSF are named Mo-MCSF and monocytes activated for a specified duration $(0-72 \mathrm{~h}$; 0-144h) with GMCSF and IL4 are Mo$\mathrm{GMCSF}^{\mathrm{IL} 4}$. To establish the relationship and transcriptional similarity between ex vivo isolated cell subsets and activated monocytes, we compared blood derived CD14 ${ }^{+}$monocytes, CD1 ${ }^{+}$DCs, CD $141^{+}$DCs (Haniffa et al., 2012), but also T- B- and NK-cells alongside CD45 ${ }^{+}$lin $^{-} H L A-D R^{\text {high }}$ lung derived cells, to Mo-MCSF, Mo-GMCSF, Mo-GMCSF ${ }^{\mathrm{IL} 4(0-72 \mathrm{~h})}$, Mo-GMCSF ${ }^{\mathrm{IL} 4(0-144 \mathrm{~h})}$ using a genome-wide approach assessing whole transcriptomes (Figure S1A).

When reducing complexity of the data by principle component analysis (PCA), T-, B-, and NK-cells formed one of three larger clusters (green), all ex vivo isolated myeloid cells formed a second large cluster (red/yellow), and both these clusters were most distinct from a third cluster containing the four ex vivo polarized monocyte-derived cell populations (blue/purple,cyan,lilac, Figure 1A, B). We validated a three-cluster structure on the genelevel by hierarchical clustering (HC) of the 1,000 most variable genes (Figure 1C, Table S1). Pearson correlation coefficient matrix (PCCM) analysis further supported this cellular relationship model (Figure 1D), clearly showing that in vitro polarized monocytes are a transcriptomically separate entity compared to ex vivo isolated human peripheral blood lymphoid and myeloid cell types.

To focus on the transcriptional analysis of mononuclear phagocyte subsets, we excluded T-, B- and NK cells from the previous dataset and used PCA to understand the transcriptional similarity between the remaining cell types (Figure 1E, S1B, C). Interestingly, all in vitro generated cells cluster separately in comparison to blood myeloid cells found in vivo, with lung-derived $\mathrm{CD}_{4} 5^{+}$lin-HLA-DR ${ }^{-}{ }^{\text {high }}$ and $\mathrm{CD} 14^{+}$monocytes forming a separate cluster away from a distinct DC cluster as well as a separate cluster for Mo-MCSF, Mo-GMCSF, Mo$\mathrm{GMCSF}^{\mathrm{IL} 4(0-144 \mathrm{~h})}$, Mo-GMCSF${ }^{\mathrm{IL} 4(0-72 \mathrm{~h})}$. Additionally, all in vitro generated monocyte-derived subsets share a common set of differentially expressed genes in comparison to ex vivo isolated $\mathrm{CD}^{+} 4^{+}$monocytes (Figure S1D, E, Table S1), clearly supporting the transcriptional difference to homeostatic cells. Consequently, these findings raise the question which cells found in vivo are represented by the monocyte model systems.

Therefore, we assessed next if the in vitro polarization models of monocytes reflect the biology of human monocyte-derived inflammatory DCs (infDCs) and inflammatory macrophages (infM) in vivo (Segura et al., 2013). To this end, we utilized a previously published dataset (GSE40484, Affymetrix $1.1^{\text {ST }}$, (Segura et al., 2013)), generated a signature 
matrix representing infDCs, infM, $\mathrm{BDCA1}^{+}(\mathrm{CD} 1 \mathrm{c}) \mathrm{DCs}, \mathrm{CD} 14^{+} \mathrm{CD} 16^{-}$monocytes and $\mathrm{CD} 14^{\mathrm{dim}} \mathrm{CD} 6^{+}$monocytes and assessed the relative enrichment of these signatures in our own dataset using linear support vector regression (Newman et al., 2015) (Figure 1F, Table S1). Mo-MCSF showed the highest enrichment of the infM-associated gene signature, while the infDC gene set was most highly enriched in Mo-GMCSF ${ }^{\mathrm{IL} 4}$, indeed suggesting that these in vitro polarization conditions reflect in vivo biology of infDCs and infM as previously shown for the mouse (Helft et al., 2015). Control gene sets derived from CD14 ${ }^{+} \mathrm{CD} 16^{-}$ monocytes were most highly enriched in ex vivo isolated $\mathrm{CD} 14^{+}$monocytes and lung-derived $\mathrm{CD}^{+} 5^{+}$lin ${ }^{-} \mathrm{HLA}-\mathrm{DR}^{\text {high }}$ cells, while the $\mathrm{BDCA}^{+}{ }^{+} \mathrm{DC}$ signature was enriched in both ex vivo isolated myeloid DC subsets of our dataset. Gene Set Enrichment Analyses (GSEA) confirmed the transcriptional similarities between infM and Mo-MCSF but also Mo-GMCSF, as well as between infDC and Mo-GMCSF ${ }^{\mathrm{IL} 4}$ (Figure S1F). Based on those observations, we defined four (corresponding) groups in both datasets, describing comparable cell subsets (see Supplementary Methods). Then, we performed Pearson coefficient correlation analysis on the gene level by comparing the expression patterns in both datasets based on the four groups. We visualized the genes with the highest correlation scores between infDC and Mo-GMCSF ${ }^{\mathrm{IL} 4}$ (072h/144h) (Figure 1G, Table S1) and between infM and both Mo-MCSF and Mo-GMCSF (Figure 1H, Table S1), which included several surface markers and secreted molecules (Figure S1G, H, I, J, Table S1). Clearly, many genes associated with activated DCs, such as CCL22, MMP12, CD226, and CCR7, were highly elevated in both infDC and Mo-GMCSF ${ }^{\mathrm{IL} 4}$ (Figure 1G), while typical macrophage genes including MARCO, CCL2 and VSIG4 were most highly expressed in infM, Mo-MCSF, and Mo-GMCSF (Figure 1H). Furthermore, differential regulation of CD226, MARCO, VSIG4, CCL2, CCL22 and MMP12 was also validated on the protein level, exemplifying the power of this computational approach (Figure 1I, J, K). Collectively, polarization of monocytes both in vivo (infDC, infM) (Segura et al., 2013) and in vitro leads to overlapping transcriptional reprogramming including many cell surface and effector molecules, which allows us to use these in vitro systems as a reductionist model to better understand the role of MCSF, GMCSF and IL4 during inflammation-induced monocyte activation.

\section{GMCSF + IL4 but not GMCSF or MCSF alone enforce a unique transcriptional signature in human $\mathrm{CD}^{+} 4^{+}$monocytes}

Next, we were interested in better understanding similarities and differences in monocyte activation induced by the three model stimuli MCSF, GMCSF and IL4. Previous work suggested significant differences between Mo-MCSF and Mo-GMCSF (Lacey et al., 2012). However, these studies did not answer the overall relationship between all three activation conditions (Figure 2A). Using the well-established surface markers CD14, CD11b and CD209, we tried to delineate differences between Mo-MCSF, Mo-GMCSF and Mo$\mathrm{GMCSF}^{\mathrm{IL} 4(0-72 \mathrm{~h})}$ (Figure S2A). This revealed that CD14 marked monocytes, Mo-MCSF and Mo-GMCSF, but not Mo-GMCSF ${ }^{\mathrm{IL} 4(0-72 \mathrm{~h})}$ as previously shown (Sallusto and Lanzavecchia, 1994). CD209 was exclusively expressed by Mo-GMCSF ${ }^{\mathrm{IL} 4(0-72 \mathrm{~h})}$. CD11b was indiscriminately expressed by all cell populations assessed. These results prompted us to 
elucidate the overall relationship between ex vivo isolated CD $14^{+}$monocytes, Mo-MCSF, MoGMCSF and Mo-GMCSF ${ }^{\mathrm{IL}(0-72 \mathrm{~h})}$ using a global transcriptomic approach (Figure S2B). PCA revealed a close relationship between Mo-MCSF and Mo-GMCSF, while monocytes and Mo$\operatorname{GMCSF}^{\mathrm{IL} 4(0-72 \mathrm{~h})}$ were clearly distinct from the two former groups (Figure 2B). This relationship was further validated on gene level by hierarchical clustering $(\mathrm{HC})$ of the 1,000 most variable genes revealing that Mo-MCSF and Mo-GMCSF formed mixed gene clusters (turquoise/light blue), marked by the high expression of macrophage related genes such as CD81, VSIG4, SIGLEC1, MARCO and FPR3 (Figure 2C, Table S2). Contrary to this, monocytes and Mo-GMCSF ${ }^{\mathrm{IL} 4(0-72 \mathrm{~h})}$ cell populations formed separated gene clusters marked by the expression of key monocyte $(A H R, S E L L, C L E C 4 D)$ or DC-associated genes $(C D 1 C$, ZBTB46), respectively. PCCM analysis confirmed these findings (Figure S2C). Gene-level analysis only including the present surfaceome of ex vivo isolated $\mathrm{CD} 14^{+}$monocytes, MoMCSF, Mo-GMCSF and Mo-GMCSF ${ }^{\mathrm{IL} 4(0-72 \mathrm{~h})}$ revealed only a small number of differentially expressed transcripts for Mo-MCSF and Mo-GMCSF populations but a markedly different expression profile of surface markers related to Mo-GMCSF ${ }^{\mathrm{IL4}(0-72 \mathrm{~h})}$ (Figure 2D). Additionally, profiling the expression of pattern recognition receptors (PRRs) expressed by ex vivo isolated $\mathrm{CD} 14^{+}$monocytes, Mo-MCSF, Mo-GMCSF and Mo-GMCSF ${ }^{\mathrm{IL} 4(0-72 \mathrm{~h})}$ revealed striking differences between these subsets (Figure S2D). Mo-MCSF, Mo-GMCSF and MoGMCSF $^{\mathrm{IL} 4(0-72 \mathrm{~h})}$ markedly downregulated the mRNA levels of important components of the inflammasome signalling complex, such as CASP1, NLRP1, 2 and 3, but upregulated the intracellular PRR NOD1. NOD2 expression was only maintained by Mo-GMCSF. Mo$\mathrm{GMCSF}^{\mathrm{IL} 4}$ (0-72h) displayed a unique set of PRRs characterized by the high expression of CD209 and CLEC10A (CD301) and the downregulation of Toll-like receptor (TLR) 7 and 5. To determine transcriptional differences, we performed co-expression network analysis based on the union of differentially expressed genes between monocytes and the three polarization conditions and mapped differential gene expression onto the network (Figure 2E, Table S2). Within the network topology, a large monocyte-related gene cluster was centrally placed surrounded by separate clusters for each of the three polarization conditions, further indicating that despite an overall very close relationship, Mo-MCSF and Mo-GMCSF are characterized by signal-specific subclusters of regulated genes (Figure 2E). Identification of differentially expressed genes between monocytes and Mo-MCSF and Mo-GMCSF further supported a close overall relationship, but also indicated significantly differently regulated genes in only one or the other condition (Figure S2E, F, Table S2). To investigate whether this would have functional implications, we performed a Gene Ontology Enrichment Analysis (GOEA) revealing that highly enriched terms in Mo-GMCSF are associated to immune response and regulation of protein metabolism, whereas most GO terms represented by Mo-MCSF are related to metabolism and G-protein coupled receptor signalling (Figure S2G, Table S2).

To identify the transcriptional regulators (TRs) involved in the generation of the monocytederived cell types, we predicted major upstream transcription factors (see Supplementary Methods) for each of the four condition-specific clusters identified in Figure 2E. We then generated a co-expression network of TRs expressed in the dataset and subsequently identified specific clusters of upregulated TRs for $\mathrm{CD}_{1} 4^{+}$monocytes (yellow), Mo-MCSF (turquoise), Mo-GMCSF (light blue) and Mo-GMCSF ${ }^{\mathrm{IL} 4(0-72 \mathrm{~h})}$ (dark blue) (Figure 2F, Table S2). Finally, we mapped the predicted master transcription factors onto the co-expression 
network and identified NFIL3, ATF4 and ETS2 among others to be specific regulators of $\mathrm{CD}_{14}{ }^{+}$monocytes. TCF 12, MEF2C and ARID3A specifically regulated the transcriptional identity of Mo-MCSF, whereas ESR1, MTF1 and SREBF1 were indicated to regulate the transcriptional makeup of Mo-GMCSF. Interestingly, RELB, which has been implicated to be important during mouse DC differentiation (Wu et al., 1998), was the only transcription factor predicted to be central to the formation of the transcriptional identity of Mo-GMCSF ${ }^{\mathrm{IL} 4(0-72 \mathrm{~h})}$, further highlighting the uniqueness of the transcriptional reprogramming induced by GMCSF and IL4.

Since the usage of traditional surface markers, such as CD14, CD11b and CD209 to discriminate in vitro polarized monocyte subsets was not very informative, we designed a comprehensive mass cytometry panel incorporating established and novel protein markers defined by our global transcriptomic approach and compared ex vivo isolated blood CD14 ${ }^{+}$ monocytes to in vitro MCSF, GMCSF, GMCSF + IL4 polarized CD14 ${ }^{+}$monocytes. Dimensionality reduction using $t$-distributed neighbour embedding ( $t$-SNE) (Maaten and Hinton, 2008) of the $\mathrm{CD} 45^{+}$lin ${ }^{-} \mathrm{HLA}-\mathrm{DR}^{+}$cell fraction comparing CD $14^{+}$monocytes, MoMCSF, Mo-GMCSF and Mo-GMCSF ${ }^{\mathrm{IL} 4(0-144 \mathrm{~h})}$ revealed donor-independent clustering into four different cellular subsets (Figure 2G, H, S2H). Overlaying their polarization history on the $t$-SNE topology revealed that the four identified clusters corresponded to the four different polarization conditions, validating the differences found within the global transcriptional data (Figure 2B). Interestingly, commonly used markers for the delineation of monocytes, macrophages and DCs, such as CD11b, CD68, CD11c and HLA-DR were expressed uniformly across all four clusters, showing that only a high-dimensional phenotyping approach enables robust detection of polarized subsets across all four polarization conditions (Figure 2H). CD14 ${ }^{+}$monocytes were characterized by a high expression of CLA and CD64, whereas Mo-GMCSF displayed a high expression of MARCO. VSIG4 was commonly expressed by Mo-GMCSF and Mo-MCSF, whereas Mo-MCSF specifically expressed high levels of the macrophage related proteins CD163, CD169 and MERTK. The Mo-GMCSF ${ }^{\mathrm{IL} 4(0-}$ 144h) cluster was characterized by specific expression of SEPP1, FceR1, CD1c and CD48. Taken together, mass cytometry enabled us to identify novel, transcriptionally validated markers, facilitating separation of different transcriptomic entities on the protein level.

\section{Functional properties of in vitro polarized monocytes}

To understand how the observed transcriptomic and phenotypic differences of Mo-MCSF, Mo-GMCSF and Mo-GMCSF ${ }^{\mathrm{IL} 4}$ translate to the functional level, we assessed their ability to phagocytize, to secrete cytokines in response to PRR stimulation, their motility and their metabolic profile.

First, we measured receptor-mediated uptake of GFP-labelled yeast or YG beads. Mo-MCSF, Mo-GMCSF and Mo-GMCSF ${ }^{\mathrm{IL} 4(0-72 \mathrm{~h})}$ were equally able to phagocytose $\mathrm{GFP}^{+}$yeast buds after 1 hour of incubation indicating that there is no differential induction of receptors and signalling pathways essentially involved in yeast uptake (Figure 3A, S3A). Mo-MCSF displayed an up to 12 times enhanced uptake of YG beads in comparison to Mo-GMCSF ${ }^{\mathrm{IL} 4(0-}$ 
72h) (Figure 3B, S3B). Therefore, despite their close relationship on the transcriptional level, these data suggest that MCSF activation but not GMCSF drives an overall phagocytic capacity in monocytes. When assessing cell motility (Figure 3C, S3C, D), Mo-MCSF, MoGMCSF and Mo-GMCSF ${ }^{\mathrm{IL} 4(0-72 \mathrm{~h})}$ cells showed very little, intermediate and high level motility, respectively. Accumulated distance and velocity analysis corroborated these findings suggesting that migratory capacity is linked to GMCSF activation, but not MCSF and is potentiated by IL4 signalling (Figure 3C, S3C, D). Metabolically, Mo-MCSF and MoGMCSF show a similar rate of oxidative phosphorylation (OXPHOS), extracellular acidification (ECAR), ATP production and glycolytic capacity (Figure 3D-E, S3E-I). Mo$\mathrm{GMCSF}^{\mathrm{IL} 4(0-72 \mathrm{~h})}$ however display a statistically significant increase of OXPHOS, ATP production and glycolytic capacity alongside an elevated maximal respiration capacity indicative of increased energetic fitness alongside higher metabolic flexibility induced by IL4specific reprogramming (Figure 3D-E, S3E-I).

Linking these functional data concerning phagocytosis, migration and metabolism back to the global transcriptomic approach, we identified key genes involved in the regulation of these processes. RAB10 (Cardoso et al., 2010), MSR1 (Bonilla et al., 2013) and DAB2 (Tumbarello et al., 2013) among others have been implicated to play a pivotal role in the regulation of phagocytosis in immune cells and subsequent antigen processing. Interestingly, these genes alongside other regulators of phagocytosis, such as RAPH1, RILPL2, TNS1 and SCARB2, are markedly upregulated on the gene level in Mo-MCSF (Figure S3J, Table S3). Similarly, regulators of migration and cell motility such as Lymphotoxin $\beta$ (LTB) (Yu et al., 2002), CCL13 (Stellato et al., 1997), CCL22 (Godiska et al., 1997) and ASAP1 (Curtis et al., 2015) were upregulated in Mo-GMCSF ${ }^{\mathrm{IL} 4(0-72 \mathrm{~h})}$ connecting transcriptomic identity of this cell population clearly with its unique functional properties (Figure S3K, Table S3). Additionally, genes involved in the regulation of glycolysis such as PFKL and PFKP were highly upregulated in Mo-GMCSF ${ }^{\mathrm{IL} 4(0-72 \mathrm{~h})}$ corresponding to the higher glycolytic capacity of these cells (Figure S3L, Table S3). Furthermore UQCRC1, SDHA, ATP5D, COX10, ATP5I genes of the respiratory chain and $I D H 3 G$, a molecule involved in the TCA cycle were highly upregulated in Mo-GMCSF ${ }^{\mathrm{IL} 4(0-72 \mathrm{~h})}$, further corroborating that the functional changes also transcend to the transcriptional level.

Finally, we stimulated Mo-MCSF, Mo-GMCSF and Mo-GMCSF ${ }^{\mathrm{IL} 4(0-72 \mathrm{~h})}$ with LPS, LPS + interferon $\gamma($ IFN- $\gamma)$, CL097 or Flagellin to evaluate their potential to secrete cytokines upon PRR ligation (Figure 3F, Table S3). Interestingly, the major immunoregulatory cytokines IL10, IL1B and MCP1were secreted only by Mo-MCSF upon activation with either of the four stimuli. This further demonstrates their similarities to in vivo infM, which have very plastic cytokine secretion properties. Conversely, IL12p70 was only secreted by Mo$\mathrm{GMCSF}^{\mathrm{IL} 4(0-72 \mathrm{~h})}$ upon stimulation with either LPS, LPS + IFN- $\gamma$ and CL097, indicating that these cells indeed possess functional overlap with DCs regarding the induction of Th1 T-cell responses through the secretion of IL12p70. IL23, a major driver of inflammatory reactions being essential for the induction of Th17 responses was only produced by cells which have been reprogrammed by GMCSF. Both Mo-GMCSF and Mo-GMCSF ${ }^{\mathrm{IL} 4(0-72 \mathrm{~h})}$ were able to produce IL23 following LPS + IFN- $\gamma$ and CL097 stimulation, respectively. This is in line with 
their similarity to ovarian cancer induced infDCs, which have been shown to have the superior capacity to induce Th17 immunity (Segura et al., 2013).

\section{IL4 regulates transcriptomic and functional polarization of moDCs and monocyte- derived "M2-like" macrophages}

Presuming that IL4 is inducing a functional convergent phenotype between monocytes and DCs, we next asked whether Mo-GMCSF ${ }^{\mathrm{IL} 4(0-144 \mathrm{~h})}$ are distinct from what was previously described as M2 macrophages, better described as monocyte-derived macrophages further activated by IL4, termed here Mo-GMCSF ${ }^{\mathrm{IL} 4(72-144 \mathrm{~h})}$. To reduce variables to a minimum, we generated Mo-GMCSF ${ }^{\mathrm{IL} 4(72-144 \mathrm{~h})}$ with GMCSF, so that the only differences to Mo$\mathrm{GMCSF}^{\mathrm{IL} 4(0-144 \mathrm{~h})}$ cells were the onset and the overall time of IL4 exposure. As controls, we also included monocytes polarized for only 3 days with either GMCSF (Mo-GMCSF ${ }^{\mathrm{IL}(\mathrm{Oh})}$ ) or GMCSF+IL4 (Mo-GMCSF ${ }^{\mathrm{IL}(0-72 \mathrm{~h})}$ ) (Figure 4A). Globally, as determined by PCA, Mo$\mathrm{GMCSF}^{\mathrm{IL} 4(72-144 \mathrm{~h})}$ were surprisingly distinct from $\left(\mathrm{Mo-GMCSF}{ }^{\mathrm{IL} 4(0-72 \mathrm{~h}),(0-144 \mathrm{~h})}\right.$ ) irrespective whether Mo-GMCSF ${ }^{\mathrm{IL} 4}$ were sampled after $72 \mathrm{~h}$ or $144 \mathrm{~h}$ of activation (Figure 4B, S4A, B). These findings were further corroborated by co-expression network analysis (Figure 4C), HC using the most variable genes (Figure 4D, Table S4), and PCCM analysis (Figure S4C). When assessing immune-phenotypes, a surprising finding was the similarly high expression of CD23 (Figure S4D), a marker formerly associated with Mo-GMCSF ${ }^{\text {IL4(72-144h) }}$ (Mantovani et al., 2002), and comparably high expression of CD209 (Figure S4E), which has been linked to monocytes with DC functionality (Geijtenbeek et al., 2000). There was no significant difference in the expression of MMP-12 (Figure S4F, G), but in the release of CCL22 (Figure S4H). In contrast, Mo-GMCSF ${ }^{\mathrm{IL} 4(0-144 \mathrm{~h})}$ showed higher uptake of yeast within 60 minutes of exposure time (Figure 4E), but similar levels of phagocytosis of YG beads (Figure S4I) when compared to Mo-GMCSF ${ }^{\mathrm{IL} 4(72-144 \mathrm{~h})}$. Mo-GMCSF ${ }^{\mathrm{IL} 4(0-144 \mathrm{~h})}$ were also more motile than Mo-GMCSF ${ }^{\mathrm{IL} 4(72-144 \mathrm{~h})}$ (Figure 4F), with both statistically higher accumulated distance (Figure S4J) and velocity (Figure S4K). Analysis of the metabolic parameters of Mo-GMCSF $^{\mathrm{IL} 4(0-144 \mathrm{~h})}$ and Mo-GMCSF ${ }^{\mathrm{IL} 4(72-144 \mathrm{~h})}$ revealed no differences in their rate of OXPHOS, ATP production and glycolysis (data not shown). Collectively, these data strongly suggest that integration of the IL4 signalling is regulated in a time-dependent manner and represents a critical checkpoint for monocyte reprogramming.

\section{Timing of IL4 stimulation determines transcriptional regulation in activated monocytes}

The experiments presented in Figure 4 allowed two alternative explanations: a dichotomous model with monocytes differentiating into monocytes with DC or macrophage functionality, or alternatively, a continuum model that integrates time of exposure suggesting significant plasticity of monocyte-derived cells. To determine which of these models described the influence of IL4 on monocytes best, we performed a time kinetics experiment, in which IL4 was added at the start of the culture $\left(\mathrm{Mo-GMCSF}^{\mathrm{IL} 4(0-144 \mathrm{~h})}\right)$, or $12\left(\mathrm{Mo}-G M C S F^{\mathrm{IL} 4(12-144 \mathrm{~h})}\right), 24$

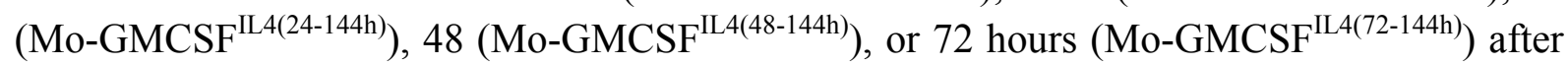


initiation of activation with GMCSF (Figure 5A). Whole transcriptomes were assessed after 144 hours and Mo-GMCSF ${ }^{\mathrm{IL} 4(0 \mathrm{~h})}$ and Mo-GMCSF ${ }^{\mathrm{IL} 4(0-72 \mathrm{~h})}$ were used as further controls. When assessing the expression of CD14 (Figure 5B, S5A) and CD209 (Figure 5C, S5B), we observed a dichotomous distribution for cells activated with IL4 being CD14 ${ }^{\text {low }}$ CD209 $9^{\text {high }}$ while cells activated only by GMCSF were $\mathrm{CD} 14^{+} \mathrm{CD} 209^{-/ \text {low }}$. In contrast, global analysis on the transcriptional level revealed a different model (Figure S5C). Applying PCA, a gradual ordering of samples formed corresponding to the exposure time to IL4, indicating a gradual commitment to a polarization state along a continuum of plasticity (Figure 5D). HC of the 1000 most variable genes showed a similar order of the samples (Figure 5E, Table S5). More importantly, this analysis suggested a gradual change in gene expression dependent on the time of IL4 exposure which was further corroborated by SOM clustering (Figure 5E). Next, we applied co-expression network analysis and mapped gene expression information for each time point onto the network (Figure 5F, Table S5). This densely populated network was characterized by two major clusters, with one being characterized by genes elevated in monocytes not exposed to IL4 (0h, red: up-regulated; blue: down-regulated). In contrast, for each time point of IL4 exposure, a different set of genes within the other major cluster was most significantly elevated (Figure 5G). Adjacent time points even showed partially overlapping gene sets strongly suggesting a plastic continuum integrating IL4 signalling over time, arguing against the dichotomous model of polarization.

Collectively, these data suggest that monocytes are reprogrammed by IL 4 signalling over time along a continuum with Mo-GMCSF ${ }^{\mathrm{IL} 4(0 \mathrm{~h})}$ and Mo-GMCSF ${ }^{\mathrm{IL} 4(72-144 \mathrm{~h})}$ being at the extreme ends.

\section{NCOR2 is a transcriptional regulator of $\mathrm{Mo-GMCSF}{ }^{\mathrm{IL} 4}$}

To better understand how IL4 enforces this unique transcriptional program in Mo$\operatorname{GMCSF}^{\mathrm{IL} 4(0-72 \mathrm{~h})}$, we generated a co-expression network by first extracting TRs from the transcriptome data expressed in either monocytes, Mo-GMCSF or monocytes activated with GMCSF and different durations of IL4 and used them as bait for building a co-expression network of TRs (Figure 6A, B). Secondly, TRs with differential expression in Mo$\mathrm{GMCSF}^{\mathrm{IL} 4(0-72 \mathrm{~h})}$ in comparison to the other cell types were filtered and thirdly, the remaining TRs were ranked based on absolute expression levels (Figure 6B, C, red: upregulated, blue: downregulated, Table S6). We identified seven TRs fulfilling these criteria with NCOR2 showing the highest expression of these TRs (Figure 6C). Analysis of NCOR2 protein expression revealed an enrichment of NCOR2 in the nucleus of Mo-GMCSF ${ }^{\mathrm{IL} 4(0-72 \mathrm{~h})}$ but not in Mo-GMCSF or monocytes (Figure 6D). Using nanostraw technology (Figure S6B), which allows viral- or liposome free siRNA delivery (Xu et al., 2014), we introduced anti-NCOR2 siRNAs for the last 24 hours of the Mo-GMCSF ${ }^{\mathrm{IL} 4(0-72 \mathrm{~h})}$ activation program. After siRNA incubation, mean NCOR2 mRNA levels in Mo-GMCSF ${ }^{\mathrm{IL} 4(0-72 \mathrm{~h})}$ were reduced to $65 \%$ relative to the control (Figure S6C), which reflects effective downregulation of NCOR2 transcription considering its long half-life of more than 24 hours (Raghavan et al., 2002). Interestingly, siRNA knockdown of NCOR2 in Mo-GMCSF ${ }^{\mathrm{IL} 4(0-72 \mathrm{~h})}$ also reduced the levels of CD209 mRNA (Figure S6D) and protein (Figure S6E). To understand the global impact of NCOR2 
on the transcriptional program of Mo-GMCSF ${ }^{\mathrm{IL} 4(0-72 \mathrm{~h})}$ and the regulation of Mo-GMCSF ${ }^{\mathrm{IL} 4}$ (0${ }^{72 h)}$ transcriptional identity by NCOR2, we performed a global transcriptome analysis of antiNCOR2 siRNA-treated Mo-GMCSF ${ }^{\mathrm{IL} 4(0-72 \mathrm{~h})}$ versus scrambled siRNA-treated MoGMCSF $^{\mathrm{IL} 4(0-72 \mathrm{~h})}$ (Figure S6F, G). We identified 1,834 variable genes after knockdown of NCOR2 (Figure S6H, Table S6). To further classify NCOR2 genes, we defined an IL4 signature based on three previously described datasets (GSE13762, GSE35304, GSE32164) with a total of 457 signature genes induced and 498 genes repressed by IL4 (Figures S6F). Overlaying these signature genes onto an expression plot comparing the previously identified variable genes between control and NCOR2 knockdown samples demonstrates that a large majority of the genes regulated by NCOR2 are IL4 signature genes (Figure 6E, Table S6). Taken together, this data establishes NCOR2 as a key regulator involved in the reprogramming of monocytes by IL4 enforcing their unique transcriptional and functional profile.

\section{Mass cytometry analysis identifies unappreciated phenotypic heterogeneity in clinically relevant Mo-GMCSF ${ }^{\mathrm{IL}(0-144 \mathrm{~h})}$ cultures}

Albeit high-dimensional protein analysis by mass cytometry clearly distinguished the four cell populations, namely monocytes, Mo-MCSF, Mo-GMCSF, and Mo-GMCSF ${ }^{\mathrm{IL} 4(0-144 \mathrm{~h})}$ (Figure 2G), we recognized a subcluster structure within the different cell subsets, which might indicate further unappreciated phenotypic heterogeneity within in vitro cultured cells. After complexity reduction, we used the Rphenograph package (Chen et al., 2016; Levine et al., 2015) to cluster the dataset and devise novel subclasses of cells within the identified clusters (Figure 7A, B). As expected, the top cluster corresponding to CD14 ${ }^{+}$monocytes did not reveal any additional heterogeneity for the tested markers and presented with a homogenous expression of the known monocyte markers CD14, CD11b, CX3CR1, alongside low expression of newly identified markers for monocyte reprogramming such as VSIG4, MARCO and SEPP1. Mo-MCSF revealed four subpopulations (cluster 2, 5, 10, 11), which were characterized by the co-expression of the tissue macrophage related markers MERTK, CD64, CD169 and CD163 and showed variable expression of L-selectin (CD62L, low in cluster 2,11) and CD26 (low in cluster 2), indicating a different migration and maturation status (Figure 7B). High-dimensional analysis of Mo-GMCSF revealed two phenotypically different subclusters within this polarized monocyte populations (cluster $7 \& 8$ ). Both clusters uniformly expressed CD14, CD68 and CD206. Albeit CD64 was expressed by clusters 7 and 8, other macrophage-related proteins, such as MERTK, CD169 and CD163, were not, further corroborating their difference from Mo-MCSF on the phenotypic level. Cluster 7additionally expressed the activation associated molecules FceR1 and CD1a, supporting a more activated status of this subpopulation of Mo-GMCSF. M-GMCSF ${ }^{\mathrm{IL} 4(0-144 \mathrm{~h})}$ showed a similar degree of heterogeneity as Mo-MCSF, however with more pronounced phenotypic differences (cluster $1,3,4,9)$. To understand these differences in more detail, we isolated Mo-GMCSF ${ }^{\mathrm{IL} 4(0-144 \mathrm{~h})}$ cells out of the dataset and analyzed these cells alone using $t$-SNE dimensionality reduction and the Rphenograph clustering algorithm (Figure 7C-E). Rphenograph revealed 11 phenotypically different subpopulations within the population polarized by GMCSF and IL4 
(Figure 7C, D). Common markers of moDC such as CD1c, CD226, CD48 and CD11c were uniformly expressed across all 11 subpopulations. Interestingly, the biggest differences across all different subpopulations were seen in the expression of activation and antigen presentation-associated molecules, such as CD1a, HLA-DR, FceR1, CD62L and CD86. Expression of these markers differed across the subpopulations with clusters 6, 1, 10, 11 showing high expression of CD1a and CD62L, whereas only cluster 5 and 2 expressed high amounts of the important co-stimulatory molecule CD86. Of note, also the expression of $\mathrm{CD} 11 \mathrm{~b}$, a marker widely used to analyze the purity of these cells in the clinic, varied significantly across the examined GMCSF + IL4 population, exemplifying the urgent need for a higher dimensional phenotyping to improve purity and therapeutic outcome when using these reprogrammed monocytes therapeutically (Figure 7E).

\section{Discussion}

Human $\mathrm{CD}_{14}^{+}$monocytes reprogrammed by exposure to either M-CSF, GM-CSF or GMCSF + IL-4 have been extensively studied as in vitro models for macrophages, "M2-like" macrophages or DCs (Akagawa et al., 2006; Randolph et al., 2008; Sallusto and Lanzavecchia, 1994; Xue et al., 2014). For example, to model DCs, moDCs (Sallusto and Lanzavecchia, 1994) were used. In the past, using these in vitro generated cells as models for macrophages and DCs was justified by morphological, phenotypical and functional similarities to in vivo found cellular subsets. However, recently it became clear that macrophages, monocytes and DCs present with very different transcriptomic make ups in vivo, reflecting their different ontogeny (Geissmann et al., 2010; Guilliams et al., 2014; Merad et al., 2013; Schlitzer et al., 2015a). Therefore, it is imperative to globally reassess the relationships between M-CSF, GM-CSF and GM-CSF + IL-4 reprogrammed monocytes and their alleged in vivo counterparts on a transcriptomic, phenotypic and functional level.

By using public data of ovarian cancer ascites associated infM and infDC (Segura et al., 2013), we revealed that M-CSF reprogrammed monocytes aligned closely to infM on the transcriptional level. This was phenotypically supported by common low expressions of CD1a and FceR1. In addition, Mo-GMCSF ${ }^{\mathrm{IL} 4}$ aligned closely to infDCs, with common phenotypic high expressions of CD1a, CD206 alongside with FceR1. Taken together, the currently available in vitro models best resemble inflammatory rather than homeostatic DC and macrophage populations, therefore, serving best as reductionist models to study the role of these cells in inflammation. For the future, we encourage to identify new culture conditions for monocyte-derived cells that resemble homeostatic DC or macrophage phenotypes guided by transcriptomic evaluation, as shown for in vitro cultures of microglia (Butovsky et al., 2013), and the identification of dedicated progenitors of DCs in the human blood and bone marrow (Breton et al., 2015; Lee et al., 2015).

Genome-wide assessments, as exemplified here on transcriptome level, allowed us to define the cellular relationships between these different model systems on a much more precise level and revealed an unexpectedly close association of monocytes differentiated by MCSF and GMCSF, while IL4 was the major driver for moDC identity. In addition, we found that 
monocytes integrate the GMCSF and IL4 signals over time, which necessitates a reassessment of our dichotomous definition of monocytes differentiating towards a macrophage or DC-like phenotype. Clearly, the varying time of onset and the variance in overall exposure to IL4 resulted in gradually changing transcriptional and functional identities of monocyte-derived cells. The unexpectedly high heterogeneity unravelled by applying high-dimensional phenotyping by mass cytometry added a further perspective of individual reactivity to the moDC response repertoire. These observations challenge our view of a strict differentiation switch between monocytes with macrophage or DC functionality induced by a single cytokine, but rather support a more dynamic differentiation model, in which cell identity is a function of nature and the duration of this input. This further corroborates that these monocyte-derived in vitro cultures model the more plastic cell types originating from monocytes during inflammation in vivo - such as infM and infDCs - than steady state macrophages or DCs.

Using our global transcriptional approach, we identified the co-repressor NCOR2 as one of the key transcriptional hubs linked to IL4-dependent differentiation of monocytes, which has not been described so far. Co-repressors such as NCOR2 play an important role throughout development and for homeostatic processes in muscle, adipose, and liver tissues (Mottis et al., 2013). NCOR 2 has been shown to be important in cell fate determination, cell differentiation and lineage progression in the nervous system (Jepsen et al., 2007), and elevated expression of NCOR2 was mainly observed in tissues with high OXPHOS activity, similar to our observations of elevated expression of NCOR2 in Mo-GMCSF ${ }^{\mathrm{IL} 4}$ (Reilly et al., 2010). Additionally, via signalling by nuclear receptors such as peroxisome proliferator-activated receptor- $\gamma$ (PPAR- $\gamma$ ) or liver X receptor (LXR), NCOR2 has been linked to the repression of NF-kB target genes in response to LPS stimulation of macrophages (Hoberg et al., 2004; Pascual et al., 2005). Nevertheless, a link from IL4-mediated signalling to NCOR2 remains elusive. Although it has been speculated that IL4 activation of human monocytes leads to endogenous PPAR- $\gamma$ ligand production (Czimmerer et al., 2012), further work is necessary to establish a link to NCOR2-mediated gene repression. Since regulation of co-repressors such as NCOR2 are tissue-, cell type- and context-dependent (Mottis et al., 2013), future studies will pinpoint, whether transcriptional control, alternative splicing, post-translational modifications, cellular localization of NCOR2, or intermediate signalling cascades or a combination thereof are involved in the molecular function of NCOR2 in IL-4IL4- driven monocyte differentiation.

In addition, we revealed unexpected heterogeneity within the monocyte cultures and finally introduce a novel set of surface markers with superior resolution on differentially stimulated monocyte cell subsets when compared to individual markers. In particular, within MoGMCSF $^{\mathrm{IL} 4}$ we identified subsets of cells that either expressed HLA-DR and CD86 or CD1a and $F c_{\varepsilon} \mathrm{R} 1$, the former representing a subpopulation with already elevated antigen presenting capacity. While induction of FceR1 on $\mathrm{CD}_{1} \mathrm{a}^{+} \mathrm{DCs}$ derived from $\mathrm{CD} 34^{+}$stem cells has been reported (Allam et al., 2004; Magerstaedt et al., 1997), this has not been studied during monocyte to Mo-GMCSF ${ }^{\mathrm{IL} 4}$ differentiation. Therefore, the heterogeneity of this event was not yet appreciated. Considering findings that $\mathrm{CD}_{1} \mathrm{a}^{+}$and $\mathrm{CD}_{1} \mathrm{a}^{-} \mathrm{Mo}-\mathrm{GMCSF}^{\mathrm{IL} 4}$ differ in their capacity to direct Th cell differentiation (Cernadas et al., 2009; Chang et al., 2000; Gogolak et 
al., 2007) a better monitoring of these cultures in a clinical setting might be beneficial for optimizing efficiency of cellular vaccines in the future. Consequently, high-dimensional characterization should be used to further optimize culture conditions, generate more homogenous cell populations, and therefore open novel avenues for optimizing cellular products derived from human monocytes for vaccination strategies.

\section{Acknowledgement}

We thank all members of the Schultze and Schlitzer Labs for critically reading the manuscript. In addition, we thank Johannes Oldenburg for providing us with buffy coats from healthy individuals. This work was supported by the German Research Foundation (SFB 832, SFB 704, INST 217/577-1 and SFB 738 (CF)), core grants of the Singapore Immunology Network (FG, EWN) and the Singapore Immunology Network immune-monitoring platform funding $(\mathrm{EWN})$. JLS, AS, WK, EL, SB, and MB are members of the Excellence Cluster ImmunoSensation. The research leading to these results has received funding from the People Program (Marie Curie Actions) of the European Union's Seventh Framework Program FP7/2077-2013 under REA grant agreement no. 317445. AS is supported by an Emmy Noether fellowship of the German research foundation (SCHL 2116/1-1) and an A*STAR BMRC Young Investigator Award. NMcG is supported by a Royal Society and Wellcome Trust grant (204464/Z/16/Z). We thank M. Ballmaier for cell sorting at MHH. 


\section{Material and Methods}

\section{Ethics statement}

Buffy coats were obtained from healthy donors in cooperation with the University hospital Bonn (local ethics vote 203/09) after written consent was given according to the Declaration of Helsinki.

\section{Isolation of human mononuclear cells from buffy coats}

Peripheral blood mononuclear cells (PBMC) were isolated by Pancoll (PAN-Biotech) density centrifugation from buffy coats. CD14-, CD56-, CD4- and CD19-specific MACS beads (Miltenyi Biotec) were used for the enrichment of $\mathrm{CD}_{14}{ }^{+}$monocytes, CD56 ${ }^{+} \mathrm{NK}$ cells, $\mathrm{CD} 4^{+}$ $\mathrm{T}$ cells and $\mathrm{CD} 19^{+} \mathrm{B}$ cells, respectively. Lung-derived monocytes were isolated from human perfusates of lung transplant recipients with informed consent and immediately sorted for $\mathrm{CD}_{4} 5^{+} \mathrm{CD} 14^{+} \mathrm{HLA}^{-D R}{ }^{\text {high }}$ cells using the FACS Fusion cell sorter (BD, USA).

\section{Generation of reprogrammed $\mathrm{CD}_{1} 4^{+}$monocytes}

$\mathrm{CD}_{14}{ }^{+}$monocytes were cultured in RPMI1640 medium supplemented with $10 \%$ FCS, $1 \%$ Penicillin-Streptomycin, 1\% Sodium pyruvate and 1\% Glutamax (all from Gibco) for three days. $\mathrm{CD} 14^{+}$monocytes were differentiated into Mo-MCSF or Mo-GMCSF in the presence of $100 \mathrm{IU} / \mathrm{ml} \mathrm{rhMCSF}$ or $800 \mathrm{IU} / \mathrm{ml} \mathrm{rhGMCSF}$, respectively. Mo-GMCSF ${ }^{\mathrm{IL} 4}$ were generated by the addition of $800 \mathrm{IU} / \mathrm{ml} \mathrm{rhGMCSF}$ and $500 \mathrm{IU} / \mathrm{ml} \mathrm{rhIL} 4$ and were incubated for up to 6 days. All cytokines were purchased from Immunotools.

\section{Flow cytometry}

Cells were washed with ice cold PBS. After FcR blockage (Miltenyi, Germany), cells were stained with the respective antibodies in PBS supplemented with 0.5\% FCS, 2.5 mM EDTA for $20 \mathrm{~min}$ at $4{ }^{\circ} \mathrm{C}$. Following antibodies were purchased from Biolegend (USA): CD19 (HIB19), CD56 (HCD56), CD11b (ICRF44), CD23 (EBVCS-5), HLA-DR (L243), CD14 (M5E2); R\&D: VSIG4 (polyclonal), CD1E (704407), MARCO (polyclonal); Becton Dickinson (BD, USA): CD209 (DCN46), CD226 (DX11); Data acquisition was performed with a LSR II (BD). Analyses were performed with FlowJo software (Tree Star).

\section{Mass cytometry}

Following culture, cells were washed with PBS (GIBCO, Life Technologies, Carlsbad, CA) and stained with cisplatin (Sigma-Aldrich, St Louis, MO) on ice to exclude dead cells. Afterwards, cells were washed with PBS containing 4\% FBS and 0.05\% Sodium azide and 
fixed with 2\% paraformaldehyde (PFA; Electron Microscopy Sciences, Hatfield, PA) overnight. Cells were permeabilized (1X perm buffer (Biolegend, San Diego, CA)) and stained with metal-conjugated antibodies (Table S7) intracellularly. Then cells were washed, metal barcoded and resuspended in water. EQ Four Element Calibration Beads (Fluidigm Corporation, South San Francisco, CA) were added at a concentration of $1 \%$ prior to acquisition. Cells were acquired and analyzed using a CyTOF1 Mass cytometer. The data was normalized (Finck et al., 2013) and events with parameters having zero values were replaced with a randomized value between 0 and -1 . Cells for each barcode were deconvoluted (using manual gating to select cells stained with two and only two barcoding channels.

\section{Mass cytometry data analysis}

Normalized mass cytometry data was exported in .fcs format and pre-processed in FlowJo Version 9.9.4 (Tree Star Inc). Pre-processing included removal of cell debris (Iridium191/193 DNA intercalating fraction) and dead cells (cisplatin ${ }^{+}$). Myeloid CD45 ${ }^{+}$lin(CD3/CD7/CD15/CD19/CD20/CD56) $)^{-}$cells were exported and used for analysis. Downstream analysis was performed using the Cytofkit $\mathrm{R}$ package. For comparison of the different reprogramming conditions data from 1000 myeloid cells was randomly sampled per donor and condition (3 donors; 12,000 cells in total) and autoLgcl-transformed including expression values for 36 surface markers (CD45, CD14, CD5, CD62L, CD48, CD68, CD66ace, CLA, HLA-DR, CD115, CD64, CD1c, FceR1, SEPP1, CD123, CD163, CXCR3, CD226, CD169, SIRP1a, Dectin1a, CD1a, CD141, MARCO, CD86, CX3CR1, CD206, VSIG4, CD88, CD34, MerTK, CD39, CD26, CD11c, CD11b, CD16). Detailed analysis on the Mo-GMCSF ${ }^{\mathrm{IL} 4}$ condition was based on 3500 cells from one individual. To define clusters of cell subpopulations, PhenoGraph method was used. Points representing individual cells in the $t$-SNE plots were color-coded to illustrate levels of protein expression or affiliations to clusters, treatment conditions or donors, respectively. Alternatively, the gplots R package was used to generate heatmaps of marker expression of individual cells or mean values over identified cell clusters. Dendrograms representing hierarchical clustering results based on the Euclidean distance measure also were included.

\section{Uptake of fluorescent microbeads or yeast}

Cells were incubated either with fluorescent monodispersed polystyrene microspheres $(1 \mu \mathrm{m}$ diameter, Fluoresbrite YG Beads, Polysciences) or yeast (GFP-expressing Pichia Pastoris ) in a cell-to-bead ratio of $1 / 10$ for $4 \mathrm{~h}$ or $60 \mathrm{~min}$ at $37^{\circ} \mathrm{C}$, respectively (Kreer et al., 2017). Afterwards, cells were harvested, washed and bead/yeast uptake was analyzed by flow cytometry using an LSR II (BD). Negative control samples were kept at $4^{\circ} \mathrm{C}$. Data analysis was performed using FlowJo software (Tree Star). 


\section{Western blot}

For hNCOR2 protein detection, the cytosolic and nuclear whole protein fractions were separated by SDS-PAGE and transferred onto a nitrocellulose membrane (Amersham) by wet blotting. Probing was performed using hNCOR2 antibody (Abcam). Signal expression values were calculated in semi-quantitative relation to HSP70 (BioTrend) and $\beta$-actin expression values (Millipore). For MMP12 protein detection, the cytosolic whole protein fractions were separated by SDS-PAGE and transferred onto a nitrocellulose membrane (Amersham) by semi-dry blotting. Probing was performed by using MMP-12 antibody (R\&D). Signal expression values were calculated in semi-quantitative relation to $\beta$-actin expression values (Millipore). All signal detection and analysis was performed via the LI-COR Odyssey system. Dignam extraction efficiency was validated for enrichment of cytosolic proteins in the cytosolic fractions compared to the nuclear proteins in nuclear fractions via HDAC1 (Cell signaling) versus GAPDH (Cell signaling) detection.

\section{Migration assay}

Migration was analyzed in $\mu$-Slide 8 well chambered coverslips (Ibidi) coated with $50 \mu \mathrm{g} / \mathrm{ml}$ human fibronectin (Alfa Aesar). $0.7 \times 10^{5}$ cells in $300 \mu 1$ VLE-RPMI (Biochrom) were transferred to each well. Live cell imaging of adherent cells was performed at $37^{\circ} \mathrm{C}$ and $5 \%$ $\mathrm{CO}_{2}$ using a fully automated inverted Olympus Fluoview 1000 confocal microscope equipped with motorized xyz stage (Märzhäuser). Cell motility was monitored over a period of $3 \mathrm{~h}$ by capture of differential interference contrast images every 5 min with a 0.40 UPLAPO $10 \mathrm{x}$ Objective (Olympus). Migration parameters were calculated using the Manual Tracking and Chemotaxis Tool plugins in ImageJ.

\section{Nanostraw-facilitated NCOR2 siRNA knockdown}

$24 \mathrm{~h}$ prior to the experiment, the nanostraw cargo chamber was washed three times with $10 \mu \mathrm{L}$ of $0.5 \%$ PEG 3500 (P3640, sigma) in PBS and equilibrated; chambers were equilibrated with $100 \mu \mathrm{L}$ RPMI 1640 media. CD14 ${ }^{+}$human monocytes were resuspended in RPMI 1640 with supplements (10\% FCS, 1\% Pen/Step, 1\% GlutaMax and 1\% NaPyruvat) and activated. siRNA solutions were prepared in 1x siRNA Buffer (Dharmacon). hNCOR2 siRNA (Dharmacon) ON Target Plus was used for knockdown. Immediately prior to the experiment, the equilibration media was removed, the tubing system was flushed with the siRNA solution or PBS only, and completely filled. Subsequently, the cell suspension was filled into the chamber and incubated for $72 \mathrm{~h}$ at $37^{\circ} \mathrm{C}$ and $5 \% \mathrm{CO}_{2}$. After $72 \mathrm{~h}$, cells were directly lysed within the chambers by adding Trizol. qRT-PCR was performed to check transfection efficiency. 


\section{Cell stimulation}

Mo-MCSF, Mo-GMCSF, Mo-GMCSF ${ }^{\mathrm{IL} 4(0-72 \mathrm{~h})}$ or Mo-GMCSF ${ }^{\mathrm{IL} 4(0-144 \mathrm{~h})}$ cells were stimulated overnight under the following conditions: Media, 100ng/ml LPS ultrapure, 100ng/ml LPS ultrapure $+1000 \mathrm{U} / \mathrm{ml} \mathrm{IFN-} \gamma, 1 \mu \mathrm{g} / \mathrm{ml}$ CL097, 100ng/ml flagellin. Supernatants were harvested after $19 \mathrm{~h}$ of stimulation and stored at $-80^{\circ} \mathrm{C}$ until further processing for cytokine analysis.

\section{Cytokine measurement}

Cytokines were measured using LEGENDplex. Briefly, diluted cell culture supernatants were incubated for 2 hours with the provided beads and detection antibodies, followed by another 30min incubation with SA-PE. After washing, beads were resuspended in washing buffer and acquired using a LSRII flow cytometer (BD). Data were analyzed with the LEGENDplex Data Analysis Software; concentration values were exported to Excel and visualized in R.

\section{Oxygen consumption rate (OCR) and ECAR measurements}

OCR and ECAR were determined using a XF-96 Extracellular Flux Analyzer (Seahorse Bioscience). For ECAR analysis the media was changed to bicarbonate-free RPMI supplemented $10 \mathrm{mM}$ glucose, $1 \mathrm{mM}$ pyruvate $\& 2 \mathrm{mM}$ glutamine $1 \mathrm{~h}$ prior to the assay and the plate was kept in a non-carbonated incubator. Measurements were performed under basal conditions and after the sequential addition of final $1 \mu \mathrm{M}$ oligomycin A, $1.5 \mu \mathrm{M}$ FCCP (fluorocarbonyl cyanide phenylhydrazone) and $0.5 \mu \mathrm{M}$ rotenone \& antimycin each. For ECAR analysis the media was changed to bicarbonate free RPMI supplemented $2 \mathrm{mM}$ glutamine $1 \mathrm{~h}$ prior to the assay and the plate was kept in a non-carbonated incubator. Measurements were performed under basal conditions and after the sequential addition of final $10 \mathrm{mM}$ glucose, 1 $\mu \mathrm{M}$ oligomycin and $100 \mathrm{mM}$ 2-Deoxyglucose. OXPHOS was calculated as (basal OCR OCR after rotenone \& antimycin treatment), ATP production was calculated as (basal OCR OCR after oligomycin A treatment), maximal respiration was calculated as (OCR after FCCP treatment - OCR after rotenone \& antimycin treatment), glycolysis was calculated as (basal ECAR - ECAR after 2-Deoxyglucose treatment), glycolytic capacity was calculated as (ECAR after oligomycin A treatment - ECAR after 2-Deoxyglucose treatment. All reagents were purchased from Sigma, except FCCP was purchased from Tocris. ECAR and OCR raw data was normalized to DNA content using the CyQuant Assay kit (Thermo Fisher).

\section{Microarray data generation}

Up to $5 \times 10^{6}$ cells were harvested and lysed in TRIzol (Invitrogen) and RNA was isolated and concentration and purity was assessed using a NanoDrop 1000 UV-Vis Spectrophotometer (Thermo Scientific). Subsequently, the TargetAmp-Nano Labeling Kit for Illumina Expression BeadChip (Epicentre) was utilized to generate biotin labeled anti-sense RNA (cRNA) according to the manufacturer's protocol. As a quality control, $100 \mathrm{ng}$ cRNA 
were reverse transcribed to cDNA and a PCR specific for $A C T B$ amplification was performed. For expression profiling, $750 \mathrm{ng}$ cRNA were hybridized to Human HT-12v3 BeadChip arrays (Illumina), stained and imaged on an Illumina iScan system.

\section{RNA-sequencing}

Total RNA was converted into libraries of double stranded cDNA molecules as a template for high throughput sequencing following the manufacturer's recommendations using the Illumina TruSeq RNA Sample Preparation Kit v2. Shortly, mRNA was purified from $100 \mathrm{ng}$ of total RNA using poly-T oligo-attached magnetic beads. Fragmentation was carried out using divalent cations under elevated temperature in Illumina proprietary fragmentation buffer. First strand cDNA was synthesized using random oligonucleotides and SuperScript II. Second strand cDNA synthesis was subsequently performed using DNA Polymerase I and RNase H. Remaining overhangs were converted into blunt ends via exonuclease/polymerase activities and enzymes were removed. After adenylation of $3^{\prime}$ ends of DNA fragments, Illumina PE adapter oligonucleotides were ligated to prepare for hybridization. DNA fragments with ligated adapter molecules were selectively enriched using Illumina PCR primer PE1.0 and PE2.0 in a 15 cycle PCR reaction. Size-selection and purification of cDNA fragments with preferentially $200 \mathrm{bp}$ in length was performed using SPRIBeads (BeckmanCoulter). Size-distribution of cDNA libraries was measured using the Agilent high sensitivity DNA assay on a Bioanalyzer 2100 system (Agilent). cDNA libraries were quantified using KAPA Library Quantification Kits (Kapa Biosystems). After cluster generation on a cBot, a 75 bp single-read run was performed on a HiSeq1500.

\section{Summary of bioinformatic analyses of microarray and RNA-Seq data}

Detailed descriptions of all bioinformatic analyses can be found in the Supplemental Information. Briefly, PCAs, PCCMs, and heatmaps of the 1000 most variable probes or genes were used to investigate the relationships between samples of the different datasets. Linear SVR (Newman et al., 2015), GSEA (Subramanian et al., 2005) and correlation analyses were applied to study the transcriptional similarities between Mo-GMCSF ${ }^{\mathrm{IL} 4}{ }^{(0-72 / 144 \mathrm{~h})}$ cells and infDCs, as well as between Mo-MCSF, Mo-GMCSF and infM (GSE40484, (Segura et al., 2013)). Genes being either commonly expressed in all monocyte-derived cells, but not in $\mathrm{CD} 14^{+}$monocytes, or in only a specific monocyte-derived subset, were determined based on differential expression analyses combined with filtering. Co-expression networks were generated either based on unions of differentially expressed genes or present TRs. GOEA was performed on genes being up- or downregulated in Mo-GMCSF in direct comparison to MoMCSF. Heatmaps of genes were created, which were linked to explored functions (migration, bead uptake and OCR/ECAR), and which recapitulated the functional outcomes in terms of magnitude. SOM Clustering was performed on all genes being present in the RNA-Seq time kinetics dataset. IL4-related gene signatures were established by comparing IL4 treated to IL4 untreated myeloid cells of the three public datasets GSE13762 (Széles et al., 2009), 
GSE35304 (Clayberger et al., 2012) and GSE32164 (Pello et al., 2012). These signatures were compared to genes being variable between siRNA anti-NCOR2 and WT RNA-Seq data samples. Microarray and RNA-Seq data were uploaded to the Gene Expression Omnibus database (www.ncbi.nlm.nih.gov/gds) and can be found under the accession number GSE96719. These signatures were compared to genes being variable between siRNA antiNCOR2 and WT RNA-Seq data samples. Microarray and RNA-Seq data were uploaded to the Gene Expression Omnibus database (www.ncbi.nlm.nih.gov/gds) and can be found under the accession number GSE96719. 


\section{Figure legends}

\section{Figure 1 Relationship of in vitro activated monocyte-derived cells.}

(A-B) PCAs based on 21,250 present probes. Displayed are principal components (PCs) (A) 1 versus 2 and (B) 1 versus 3. (C) Heatmap of the top 1000 genes being most variable across the dataset. $\log _{2}$-expression values were z-transformed and scaled (-2 (blue) to 2 (red)). (D) Heatmap visualizing Pearson correlation values (PCV) calculated pairwise between all cell types on the basis of the top 1000 most variable genes. (E) PCA based on 23,952 present probes. (F) Relative fractions of monocyte, BDCA1 $1^{+} \mathrm{DC}$, infM and infDC gene signatures in $\mathrm{CD}_{1}{ }^{+}$monocytes, different monocyte-derived cells and DCs. (G-H) Heatmaps of genes specifically expressed in (G) infDCs compared to infM, BDCA1 ${ }^{+}$DCs and monocytes (dataset 1 ), and in Mo-GMCSF ${ }^{\mathrm{IL}(0-72 \mathrm{~h})}$ cells compared to all other investigated monocytederived cells, CD14 $4^{+}$monocytes and DCs (dataset 2), or in $(\mathbf{H})$ infM compared to infDCs, $\mathrm{BDCA}^{+}$DCs and monocytes (dataset 1), and in Mo-GMCSF and Mo-MCSF compared to Mo-GMCSF ${ }^{\mathrm{IL} 4(0-72 \mathrm{~h})}, \mathrm{CD}^{+} 4^{+}$monocytes and DCs (dataset 2). PCVs were calculated between the indicated group patterns of dataset 1 versus dataset 2 and are displayed as a barplot next to the heatmaps. Only genes with a correlation $>0.4$ are displayed. Genes further analyzed in Figure $1 \mathrm{I}-\mathrm{K}$ are highlighted in red. $\log _{2}$-expression values were $\mathrm{z}$-transformed and scaled (-2 (blue) to 2 (red)). (I) Histograms show relative expression of CD226, MARCO and VSIG4 on $\mathrm{CD}^{+} 4^{+}$monocytes, Mo-MCSF, Mo-GMCSF and Mo-GMCSF ${ }^{\mathrm{IL} 4(0-72 \mathrm{~h})}$ analyzed by flow cytometry. Representative data from four different donors is shown. (J) Analysis of cell culture supernatants of Mo-MCSF, Mo-GMCSF and Mo-GMCSF ${ }^{\mathrm{IL}(0-72 \mathrm{~h})}$ for CCL22 and CCL2 using ELISA ( $\mathrm{n}=3$ with 2 technical replicates each, mean + SEM). Statistical significance was determined using one-way RM (repeated measures) ANOVA and Tukey's method for multiple test correction, with $* p<0.05,{ }^{* *} p<0.01$, and $* * * p<0.001$. n.d. $=$ not detected. (K) Relative quantification of MMP12 in CD14 monocytes, Mo-MCSF, MoGMCSF and Mo-GMCSF ${ }^{\mathrm{IL} 4(0-72 \mathrm{~h})}$ using Western blot $(\mathrm{n}=3$, mean + SEM). Statistical significance was determined using one-way ANOVA and Tukey's method for multiple test correction, with $* p<0.05$.

\section{Figure 2 Mo-GMCSF ${ }^{\mathrm{IL} 4}$ are most distinct from Mo-MCSF and Mo-GMCSF.}

(A) Schema describing the biological questions addressed in Figure 2 and S2. (B) PCA based on 18,318 present probes. (C) Heatmap of the top 1000 genes being most variable across the dataset. $\log _{2}$-expression values were z-transformed and scaled (-2 (blue) to 2 (red)). (D) Heatmap of genes being specifically expressed in a single out of the three monocyte-derived cell types, compared to $\mathrm{CD} 14^{+}$monocytes as well as to the other two monocyte-derived cell types. $\log _{2}$-expression values were z-transformed and scaled (-1.5 (blue) to 1.5 (red)). (E) Co-expression networks based on the union of 2,086 differentially expressed genes (FoldChange $>2$ or $<-2$ and FDR-adjusted $p$-value $<0.05$ ) between each of the three types of monocyte-derived cells compared to $\mathrm{CD} 14^{+}$monocytes. The Fold-Change of the respective cell type compared to the overall mean was mapped onto the networks and displayed in colors ranging from blue (negative Fold-Change) over white to red (positive Fold-Change). Based on the Fold-Change patterns, the networks were divided into four main clusters, each cluster representing one of the four cell types, respectively. (F) Co-expression network based on 411 
TRs expressed in the dataset containing CD14 ${ }^{+}$monocytes, Mo-MCSF, Mo-GMCSF and Mo$\operatorname{GMCSF}^{\mathrm{IL} 4(0-72 \mathrm{~h})}$. For each cell type, the Fold-Change of the respective cell type compared to the overall mean was mapped onto the network. Cell type-specific clusters of upregulated regulators were generated, indicated by the color-coded shadings behind the network. TRs highlighted in red were predicted to be unique master regulators for the corresponding cell type. The prediction was performed on all genes being highlighted in red (upregulated in the cell type compared to the overall mean with a Fold-Change $>1.5$ ) in the corresponding cell type-specific cluster in E. Master regulators, which were identified for more than one cell type, were excluded. (G) $t$-SNE composite dimensions (tsne1 and 2) of CD14 $4^{+}$monocytes, Mo-MCSF, Mo-GMCSF and Mo-GMCSF ${ }^{\mathrm{IL} 4(0-144 \mathrm{~h})}$ analyzed by mass cytometry ( 3 intermixed donors shown). (H) Heatmap and hierarchical clustering of mean surface marker expression analyzed using mass cytometry on $\mathrm{CD} 14^{+}$monocytes, Mo-MCSF, Mo-GMCSF and Mo$\mathrm{GMCSF}^{\mathrm{IL} 4(0-144 \mathrm{~h})}$. Normalized intensity values were z-transformed and scaled (-6 (blue) to 6 (red)). Color code depicts cluster assignment according to culture condition. Colour code as in G.

\section{Figure 3 Prediction of activated monocyte functionality.}

(A) Flow cytometric analysis of Mo-MCSF, Mo-GMCSF and Mo-GMCSF ${ }^{\mathrm{IL} 4(0-72 \mathrm{~h})}$ after $1 \mathrm{~h}$ of incubation with GFP-expressing yeast (histogram: 1 representative of 3 replicates, bar plot $\mathrm{n}=3$, mean + SEM). Statistical significance between cell types after $1 \mathrm{~h}$ of incubation was determined using one-way RM ANOVA, with $(p>0.05)$. (B) Flow cytometric analysis of Mo-MCSF, Mo-GMCSF and Mo-GMCSF ${ }^{\mathrm{IL} 4(0-72 \mathrm{~h})}$ after $4 \mathrm{hrs}$ of incubation with YG beads $(n=5-6$, mean + SEM). Statistical significance between cell types after $4 \mathrm{~h}$ of incubation was determined using one-way RM ANOVA and Tukey's method for multiple test correction, with $* * * p<0.001$. (C) Migration tracks of Mo-MCSF, Mo-GMCSF and Mo-GMCSF ${ }^{\mathrm{IL} 4(0-72 \mathrm{~h})}$ migrating on a surface coated with fibronectin for 3 hrs. Results show one representative experiment out of three. (D) OCR of Mo-MCSF, Mo-GMCSF and Mo-GMCSF ${ }^{\mathrm{IL} 4(0-72 \mathrm{~h})}$, followed by sequential addition of oligomycin, FCCP, and Rot/AA. (E) ECAR of Mo-MCSF, Mo-GMCSF and Mo-GMCSF ${ }^{\mathrm{IL} 4(0-72 \mathrm{~h})}$, followed by sequential addition of glucose, oligomycin and 2-Deoxyglucose. (F) Heatmap displaying mean secreted cytokine concentrations of up to four donors. Expression values were z-transformed and scaled ( -3 (blue) to 3 (red)). Raw data can be found in Table S3.

Figure 4 Mo-GMCSF ${ }^{\mathrm{IL4(0-144h)}}$ differ from Mo-GMCSF ${ }^{\mathrm{IL} 4(72-144 \mathrm{~h})}$ monocyte-derived cells. (A) Schema describing the biological questions addressed in Figure 4 and S4. (B) PCA based on 18,857 present probes. (C) Co-expression network describing the relationships between all samples of the dataset containing $\mathrm{CD}_{1} 4^{+}$monocytes and four types of monocyte-derived cells based on 13,691 present genes. (D) Heatmap of the top 1000 genes being most variable across the dataset. $\log _{2}$-expression values were z-transformed and scaled (-2 (blue) to 2 (red)). Genes were grouped together (black boxes), dependent on in which cell type(s) they appeared as highly expressed. The corresponding group-related cell types are highlighted on the left side next to the heatmap (color coded). Important genes of each cluster are depicted on the right side next to the heatmap. (E) Flow cytometric analysis of Mo-GMCSF ${ }^{\mathrm{IL4} 472-144 \mathrm{~h})}$ and Mo- 
$\mathrm{GMCSF}^{\mathrm{IL} 4(0-144 \mathrm{~h})}$ after $1 \mathrm{~h}$ of incubation with GFP-expressing yeast ( $\mathrm{n}=4-6$, mean + SEM). Statistical significance between cell types after $1 \mathrm{~h}$ of incubation was determined using Student's t-test with $*_{p}<0.05$. (F) Migration tracks of Mo-GMCSF ${ }^{\mathrm{IL4}(72-144 \mathrm{~h})}$ and Mo$\operatorname{GMCSF}^{\mathrm{IL} 4(0-144 \mathrm{~h})}$ migrating on a surface coated with fibronectin for $3 \mathrm{hrs}$. Results show one representative experiment out of three.

Figure 5 Timing of IL4 determines transcriptional regulation in activated monocytes.

(A) Schema describing the approach of the time kinetic experiment. (B-C) Histograms show relative expression of CD14 and CD209 on the depicted culture timings analyzed by flow cytometry. (D) PCA based on 12,794 present genes. (E) Heatmap of the top 1000 genes being most variable across the dataset. $\log _{2}$-expression values were $\mathrm{z}$-transformed and scaled (-2 (blue) to 2 (red)). Below SOM-clustering, determined based on the expression profiles of the 12,794 present genes across all cell types. (F) Co-expression networks based on the union of 2,775 genes being differentially expressed (Fold-Change $>1.5$ or $<-1.5$ and FDR-corrected pvalue $<0.05)$ between each monocyte-derived cell type activated by IL4 compared to MoGMCSF. For each cell type, the Fold-Change of the respective cell type compared to the overall mean was mapped onto the networks and displayed in blue (Fold-Change $<=1.5$ ) or red (Fold-Change $>=1.5$ ). (G) Examples of genes located in the condition-related clusters depicted in $\mathbf{F}$ and in the first column, having a Fold-Change $>=1.5$ for the corresponding condition. Genes listed in the second column are condition-specific, genes displayed in the following columns are shared between the clusters of two consecutive time points.

\section{Figure 6 NCOR2 is a transcriptional regulator of Mo-GMCSF ${ }^{\mathrm{IL} 4(0-72 / 144 \mathrm{~h})}$.}

(A-B) Co-expression networks based on 267 TRs being expressed in CD14 ${ }^{+}$monocytes and four types of monocyte-derived cells. For (A) Mo-GMCSF and (B) Mo-GMCSF ${ }^{\mathrm{IL} 4(0-72 \mathrm{~h})}$, the fold-change compared to $\mathrm{CD} 14^{+}$monocytes was mapped onto the network. According to the resulting patterns, a Mo-GMCSF ${ }^{\mathrm{IL}(0-72 \mathrm{~h})}$-specific cluster of upregulated regulators was generated, which is indicated by the color-coded shading (dark blue) behind the network. Upregulated regulators in Mo-GMCSF ${ }^{\mathrm{IL} 4(0-72 \mathrm{~h})}$ (as determined in C) within the network are labeled in black. (C) Heatmap of TRs, which were identified to be specifically upregulated in Mo-GMCSF $^{\mathrm{IL} 4(0-72 \mathrm{~h})}$ and Mo-GMCSF ${ }^{\mathrm{IL} 4(0-144 \mathrm{~h})}$ cells compared to CD14 $4^{+}$monocytes and MoGMCSF. $\log _{2}$-expression values were $\mathrm{z}$-transformed and scaled (-1.15 (blue) to 1.15 (red)). (D) Relative quantification of NCOR2 protein in cytoplasm and nucleus of CD14 ${ }^{+}$monocytes, Mo-GMCSF and Mo-GMCSF ${ }^{\mathrm{IL}(0-72 \mathrm{~h})}$ using Western blot alongside HSP70 and $\beta$-Actin ( $\mathrm{n}=3$, mean + SEM). (E) Scatterplot of 1,834 variable genes across the dataset containing cells with siRNA $\alpha$ NCoR2 (y-axis) and control cells with scrambled RNA (x-axis). Displayed are $\log _{2}-$ mean expression values. Highlighted genes were determined (as described in Figure S6F) to be either induced (red) or repressed (blue) by IL4 in external datasets. 
Figure 7 Mass cytometry analysis identifies unappreciated phenotypic heterogeneity in clinically relevant Mo-GMCSF ${ }^{\mathrm{IL}(0-144 \mathrm{~h})}$ cultures

(A) Phenograph analysis of CD14 $4^{+}$monocytes, Mo-MCSF, Mo-GMCSF and Mo-GMCSF ${ }^{\mathrm{IL} 4}$ (0-144h) based on mass cytometry expression data derived from three donors (as presented in Figure 2G) including 36 myeloid-related surface markers. Affiliation of individual cells to the 11 identified clusters is indicated by color coding and visualized in a $t$-SNE plot. (B) Heatmap and hierarchical clustering of mean surface marker expression of the 11 individual clusters identified in (A). Reprogramming conditions matching the clusters according to A and Figure $2 \mathrm{G}$ are indicated on the right. (C) Mass cytometry analysis focusing on $3500 \mathrm{Mo}-\mathrm{GMCSF}^{\mathrm{IL} 4(0-}$ ${ }^{144 h)}$ cells derived from one representative donor analyzed using Phenograph and visualized in a $t$-SNE plot. (D) Heatmap and hierarchical clustering of mean surface marker expression of the 11 individual clusters identified in (C). (E) Feature plot display of the expression of selected surface markers (CD1a, FceR1, HLA-DR, CD5, CD11b, CD86) in Mo-GMCSF ${ }^{\mathrm{IL} 4(0-}$ ${ }^{144 \mathrm{~h})}$ cells overlaid onto the $t$-SNE plot. 


\section{References}

Akagawa, K.S., Komuro, I., Kanazawa, H., Yamazaki, T., Mochida, K., Kishi, F., 2006. Functional heterogeneity of colony-stimulating factor-induced human monocyte-derived macrophages. Respirology 11 Suppl, S32-6.

Allam, J.-P., Klein, E., Bieber, T., Novak, N., 2004. Transforming growth factor-beta1 regulates the expression of the high-affinity receptor for IgE on CD34 stem cell-derived CD1a dendritic cells in vitro. The Journal of investigative dermatology 123, 676-682.

Auffray, C., Sieweke, M.H., Geissmann, F., 2009. Blood monocytes: development, heterogeneity, and relationship with dendritic cells. Annual review of immunology 27, 669-692.

Bonilla, D.L., Bhattacharya, A., Sha, Y., Xu, Y., Xiang, Q., Kan, A., Jagannath, C., Komatsu, M., Eissa, N.T., 2013. Autophagy Regulates Phagocytosis by Modulating the Expression of Scavenger Receptors. Immunity 39, 537-547.

Breton, G., Lee, J., Zhou, Y.J., Schreiber, J.J., Keler, T., Puhr, S., Anandasabapathy, N., Schlesinger, S., Caskey, M., Liu, K., Nussenzweig, M.C., 2015. Circulating precursors of human CD1c+ and CD141+ dendritic cells. The Journal of experimental medicine 207, $1273-413$.

Butovsky, O., Jedrychowski, M.P., Moore, C.S., Cialic, R., Lanser, A.J., Gabriely, G., Koeglsperger, T., Dake, B., Wu, P.M., Doykan, C.E., Fanek, Z., Liu, L., Chen, Z., Rothstein, J.D., Ransohoff, R.M., Gygi, S.P., Antel, J.P., Weiner, H.L., 2013. Identification of a unique TGF- $\beta$-dependent molecular and functional signature in microglia. Nat. Neurosci. 17, 131-143.

Cardoso, C.M.P., Jordao, L., Vieira, O.V., 2010. Rab10 Regulates Phagosome Maturation and Its Overexpression Rescues Mycobacterium-Containing Phagosomes Maturation. Traffic $11,221-235$.

Cella, M., Döhring, C., Samaridis, J., Dessing, M., Brockhaus, M., Lanzavecchia, A., Colonna, M., 1997. A novel inhibitory receptor (ILT3) expressed on monocytes, macrophages, and dendritic cells involved in antigen processing. The Journal of experimental medicine $185,1743-1751$.

Cernadas, M., Lu, J., Watts, G., Brenner, M.B., 2009. CD1a expression defines an interleukin12 producing population of human dendritic cells. Clin. Exp. Immunol. 155, 523-533.

Chang, C.C., Wright, A., Punnonen, J., 2000. Monocyte-derived CD1a+ and CD1a- dendritic cell subsets differ in their cytokine production profiles, susceptibilities to transfection, and capacities to direct Th cell differentiation. Journal of immunology 165, 3584-3591.

Chen, H., Lau, M.C., Wong, M.T., Newell, E.W., Poidinger, M., Chen, J., 2016. Cytofkit: A Bioconductor Package for an Integrated Mass Cytometry Data Analysis Pipeline. PLoS Comput Biol 12, e1005112.

Clayberger, C., Finn, M.W., Wang, T., Saini, R., Wilson, C., Barr, V.A., Sabatino, M., 
Castiello, L., Stroncek, D., Krensky, A.M., 2012. 15 kDa granulysin causes differentiation of monocytes to dendritic cells but lacks cytotoxic activity. Journal of immunology 188, 6119-6126.

Curtis, J., Luo, Y., Zenner, H.L., Cuchet-Lourenço, D., Wu, C., Lo, K., Maes, M., Alisaac, A., Stebbings, E., Liu, J.Z., Kopanitsa, L., Ignatyeva, O., Balabanova, Y., Nikolayevskyy, V., Baessmann, I., Thye, T., Meyer, C.G., Nürnberg, P., Horstmann, R.D., Drobniewski, F., Plagnol, V., Barrett, J.C., Nejentsev, S., 2015. Susceptibility to tuberculosis is associated with variants in the ASAP1 gene encoding a regulator of dendritic cell migration. Nat. Genet. 47, 523-527.

Czimmerer, Z., Varga, T., Póliska, S., Nemet, I., Szanto, A., Nagy, L., 2012. Identification of novel markers of alternative activation and potential endogenous PPAR $\gamma$ ligand production mechanisms in human IL-4 stimulated differentiating macrophages. Immunobiology 217, 1301-1314.

Dinter, J., Gourdain, P., Lai, N.Y., Duong, E., Bracho-Sanchez, E., Rucevic, M., Liebesny, P.H., Xu, Y., Shimada, M., Ghebremichael, M., Kavanagh, D.G., Le Gall, S., 2014. Different antigen-processing activities in dendritic cells, macrophages, and monocytes lead to uneven production of HIV epitopes and affect CTL recognition. Journal of immunology 193, 4322-4334.

Fehlings, M., Drobbe, L., Moos, V., Renner Viveros, P., Hagen, J., Beigier-Bompadre, M., Pang, E., Belogolova, E., Churin, Y., Schneider, T., Meyer, T.F., Aebischer, T., Ignatius, R., 2012. Comparative analysis of the interaction of Helicobacter pylori with human dendritic cells, macrophages, and monocytes. Infection and Immunity 80, 2724-2734.

Finck, R., Simonds, E.F., Jager, A., Krishnaswamy, S., Sachs, K., Fantl, W., Pe'er, D., Nolan, G.P., Bendall, S.C., 2013. Normalization of mass cytometry data with bead standards. Cytometry A 83, 483-494.

Fraser, D.A., Laust, A.K., Nelson, E.L., Tenner, A.J., 2009. C1q differentially modulates phagocytosis and cytokine responses during ingestion of apoptotic cells by human monocytes, macrophages, and dendritic cells. Journal of immunology 183, 6175-6185.

Frucht, D.M., Aringer, M., Galon, J., Danning, C., 2000. Stat4 is expressed in activated peripheral blood monocytes, dendritic cells, and macrophages at sites of Th1-mediated inflammation. The Journal of Immunology 164, 4659-4664.

Geijtenbeek, T.B., Torensma, R., van Vliet, S.J., van Duijnhoven, G.C., Adema, G.J., van Kooyk, Y., Figdor, C.G., 2000. Identification of DC-SIGN, a novel dendritic cell-specific ICAM-3 receptor that supports primary immune responses. Cell 100, 575-585.

Geissmann, F., Manz, M.G., Jung, S., Sieweke, M.H., Merad, M., Ley, K., 2010. Development of monocytes, macrophages, and dendritic cells. Science 327, 656-661.

Ginhoux, F., Guilliams, M., 2016. Tissue-Resident Macrophage Ontogeny and Homeostasis. Immunity 44, 439-449.

Ginhoux, F., Jung, S., 2014. Monocytes and macrophages: developmental pathways and tissue homeostasis. Nature reviews. Immunology 14, 392-404. 
Godiska, R., Chantry, D., Raport, C.J., Sozzani, S., Allavena, P., Leviten, D., Mantovani, A., Gray, P.W., 1997. Human Macrophage-derived Chemokine (MDC), a Novel Chemoattractant for Monocytes, Monocyte-derived Dendritic Cells, and Natural Killer Cells. Journal of Experimental Medicine 185, 1595-1604.

Gogolak, P., Rethi, B., Szatmari, I., Lanyi, A., Dezso, B., Nagy, L., Rajnavolgyi, E., 2007. Differentiation of CD1a- and CD1a+ monocyte-derived dendritic cells is biased by lipid environment and PPARgamma. Blood 109, 643-652.

Grajales-Reyes, G.E., Iwata, A., Albring, J.O.R., Wu, X., Tussiwand, R., KC, W., Kretzer, N.M., o, C.G.B.N., Durai, V., Bagadia, P., Haldar, M., nheit, J.O.R.S.O., Rosenbauer, F., Murphy, T.L., Murphy, K.M., 2015. Batf3 maintains autoactivation of Irf8 for commitment of a CD8\&alpha;+ conventional DC clonogenic progenitor. Nature immunology 1-12.

Guilliams, M., Ginhoux, F., Jakubzick, C., Naik, S.H., Onai, N., Schraml, B.U., Segura, E., Tussiwand, R., Yona, S., 2014. Dendritic cells, monocytes and macrophages: a unified nomenclature based on ontogeny. Nature reviews. Immunology 14, 571-578.

Guilliams, M., van de Laar, L., 2015. A Hitchhiker's Guide to Myeloid Cell Subsets: Practical Implementation of a Novel Mononuclear Phagocyte Classification System. Front Immunol 6, 1-12.

Haniffa, M., Shin, A., Bigley, V., McGovern, N., Teo, P., See, P., Wasan, P.S., Wang, X.N., Malinarich, F., Malleret, B., Larbi, A., Tan, P., Zhao, H., Poidinger, M., Pagan, S., Cookson, S., Dickinson, R., Dimmick, I., Jarrett, R.F., Renia, L., Tam, J., Song, C., Connolly, J., Chan, J.K., Gehring, A., Bertoletti, A., Collin, M., Ginhoux, F., 2012. Human tissues contain CD141hi cross-presenting dendritic cells with functional homology to mouse CD103+ nonlymphoid dendritic cells. Immunity 37, 60-73.

Heidkamp, G.F., Sander, J., Lehmann, C.H.K., Heger, L., Eissing, N., Baranska, A., Lühr, J.J., Hoffmann, A., Reimer, K.C., Lux, A., Söder, S., Hartmann, A., Zenk, J., Ulas, T., McGovern, N., Alexiou, C., Spriewald, B., Mackensen, A., Schuler, G., Schauf, B., Forster, A., Repp, R., Fasching, P.A., Purbojo, A., Cesnjevar, R., Ullrich, E., Ginhoux, F., Schlitzer, A., Nimmerjahn, F., Schultze, J.L., Dudziak, D., 2016. Human lymphoid organ dendritic cell identity is predominantly dictated by ontogeny, not tissue microenvironment. Science Immunology 1, eaai7677-eaai7677.

Helft, J., Böttcher, J., Chakravarty, P., Zelenay, S., Huotari, J., Schraml, B.U., Goubau, D., Sousa, C.R.E., 2015. GM-CSF Mouse Bone Marrow Cultures Comprise a Heterogeneous Population of CD11c+MHCII+ Macrophages and Dendritic Cells. Immunity 42, 11971211.

Hoberg, J.E., Yeung, F., Mayo, M.W., 2004. SMRT derepression by the IkappaB kinase alpha: a prerequisite to NF-kappaB transcription and survival. Molecular Cell 16, 245255 .

Jepsen, K., Solum, D., Zhou, T., McEvilly, R.J., Kim, H.-J., Glass, C.K., Hermanson, O., Rosenfeld, M.G., 2007. SMRT-mediated repression of an H3K27 demethylase in progression from neural stem cell to neuron. Nature 450, 415-419. 
Kreer, C., Kuepper, J.M., Zehner, M., Quast, T., Kolanus, W., Schumak, B., Burgdorf, S., 2017. N-glycosylation converts non-glycoproteins into mannose receptor ligands and reveals antigen-specific $\mathrm{T}$ cell responses in vivo. Oncotarget 8, 6857-6872.

Krutzik, S.R., Tan, B., Li, H., Ochoa, M.T., Liu, P.T., Sharfstein, S.E., Graeber, T.G., Sieling, P.A., Liu, Y.-J., Rea, T.H., Bloom, B.R., Modlin, R.L., 2005. TLR activation triggers the rapid differentiation of monocytes into macrophages and dendritic cells. Nat. Med. 11, 653-660.

Lacey, D.C., Achuthan, A., Fleetwood, A.J., Dinh, H., Roiniotis, J., Scholz, G.M., Chang, M.W., Beckman, S.K., Cook, A.D., Hamilton, J.A., 2012. Defining GM-CSF- and macrophage-CSF-dependent macrophage responses by in vitro models. Journal of immunology 188, 5752-5765.

Lee, J., Breton, G., Oliveira, T.Y.K., Zhou, Y.J., Aljoufi, A., Puhr, S., Cameron, M.J., Sékaly, R.-P., Nussenzweig, M.C., Liu, K., 2015. Restricted dendritic cell and monocyte progenitors in human cord blood and bone marrow. The Journal of experimental medicine 404, 193-399.

Levine, J.H., Simonds, E.F., Bendall, S.C., Davis, K.L., Amir, E.-A.D., Tadmor, M.D., Litvin, O., Fienberg, H.G., Jager, A., Zunder, E.R., Finck, R., Gedman, A.L., Radtke, I., Downing, J.R., Pe'er, D., Nolan, G.P., 2015. Data-Driven Phenotypic Dissection of AML Reveals Progenitor-like Cells that Correlate with Prognosis. Cell 1-15.

Maaten, L.V.D., Hinton, G., 2008. Visualizing Data using t-SNE. Journal of Machine Learning Research 9, 2579-2605.

Magerstaedt, R., Kraft, S., Strobel, I., Jürgens, M., Hanau, D., Wessendorf, J., Bieber, T., 1997. Induction of Fc epsilon RI alpha mRNA and protein synthesis by interleukin 4 in CD34+ cells-derived CD1a+ dendritic cells. Adv. Exp. Med. Biol. 417, 353-355.

Mantovani, A., Sozzani, S., Locati, M., Allavena, P., Sica, A., 2002. Macrophage polarization: tumor-associated macrophages as a paradigm for polarized M2 mononuclear phagocytes. Trends Immunol. 23, 549-555.

Merad, M., Sathe, P., Helft, J., Miller, J., Mortha, A., 2013. The dendritic cell lineage: ontogeny and function of dendritic cells and their subsets in the steady state and the inflamed setting. Annual review of immunology 31, 563-604.

Mottis, A., Mouchiroud, L., Auwerx, J., 2013. Emerging roles of the corepressors NCoR1 and SMRT in homeostasis. Genes \& Development 27, 819-835.

Murray, P.J., Allen, J.E., Biswas, S.K., Fisher, E.A., Gilroy, D.W., Goerdt, S., Gordon, S., Hamilton, J.A., Ivashkiv, L.B., Lawrence, T., Locati, M., Mantovani, A., Martinez, F.O., Mege, J.-L., Mosser, D.M., Natoli, G., Saeij, J.P., Schultze, J.L., Shirey, K.A., Sica, A., Suttles, J., Udalova, I., van Ginderachter, J.A., Vogel, S.N., Wynn, T.A., 2014. Macrophage Activation and Polarization: Nomenclature and Experimental Guidelines. Immunity 41, 14-20.

Newman, A.M., Liu, C.L., Green, M.R., Gentles, A.J., Feng, W., Xu, Y., Hoang, C.D., Diehn, 
M., Alizadeh, A.A., 2015. Robust enumeration of cell subsets from tissue expression profiles. Nat Meth 12, 453-457.

Ohradanova-Repic, A., Machacek, C., Fischer, M.B., Stockinger, H., 2016. Differentiation of human monocytes and derived subsets of macrophages and dendritic cells by the HLDA10 monoclonal antibody panel. Clin Transl Immunology 5, e55.

Pascual, G., Fong, A.L., Ogawa, S., Gamliel, A., Li, A.C., Perissi, V., Rose, D.W., Willson, T.M., Rosenfeld, M.G., Glass, C.K., 2005. A SUMOylation-dependent pathway mediates transrepression of inflammatory response genes by PPAR-gamma. Nature 437, 759-763.

Pello, O.M., De Pizzol, M., Mirolo, M., Soucek, L., Zammataro, L., Amabile, A., Doni, A., Nebuloni, M., Swigart, L.B., Evan, G.I., Mantovani, A., Locati, M., 2012. Role of cMYC in alternative activation of human macrophages and tumor-associated macrophage biology. Blood 119, 411-421.

Perdiguero, E.G., Geissmann, F., 2016. The development and maintenance of resident macrophages. Nature immunology 17, 2-8.

Raghavan, A., Ogilvie, R.L., Reilly, C., Abelson, M.L., Raghavan, S., Vasdewani, J., Krathwohl, M., Bohjanen, P.R., 2002. Genome-wide analysis of mRNA decay in resting and activated primary human T lymphocytes. Nucleic Acids Res. 30, 5529-5538.

Randolph, G.J., Jakubzick, C., Qu, C., 2008. Antigen presentation by monocytes and monocyte-derived cells. Current Opinion in Immunology 20, 52-60.

Reilly, S.M., Bhargava, P., Liu, S., Gangl, M.R., Gorgun, C., Nofsinger, R.R., Evans, R.M., Qi, L., Hu, F.B., Lee, C.-H., 2010. Nuclear receptor corepressor SMRT regulates mitochondrial oxidative metabolism and mediates aging-related metabolic deterioration. Cell Metab. 12, 643-653.

Rossi, R., Lichtner, M., De Rosa, A., Sauzullo, I., Mengoni, F., Massetti, A.P., Mastroianni, C.M., Vullo, V., 2011. In vitro effect of anti-human immunodeficiency virus CCR5 antagonist maraviroc on chemotactic activity of monocytes, macrophages and dendritic cells. Clin. Exp. Immunol. 166, 184-190.

Sallusto, F., Lanzavecchia, A., 1994. Efficient presentation of soluble antigen by cultured human dendritic cells is maintained by granulocyte/macrophage colony-stimulating factor plus interleukin 4 and downregulated by tumor necrosis factor alpha. The Journal of experimental medicine 179, 1109-1118.

Satpathy, A.T., Wu, X., Albring, J.C., Murphy, K.M., 2012. Re(de)fining the dendritic cell lineage. Nature immunology 13, 1145-1154.

Schlitzer, A., Ginhoux, F., 2014. Organization of the mouse and human DC network. Current Opinion in Immunology 26, 90-99.

Schlitzer, A., McGovern, N., Ginhoux, F., 2015a. Dendritic cells and monocyte-derived cells: Two complementary and integrated functional systems. Semin. Cell Dev. Biol. 
Schlitzer, A., Sivakamasundari, V., Chen, J., Bin Sumatoh, H.R., Schreuder, J., Lum, J., Malleret, B., Zhang, S., Larbi, A., Zolezzi, F., Renia, L., Poidinger, M., Naik, S., Newell, E.W., Robson, P., Ginhoux, F., 2015b. Identification of cDC1- and cDC2-committed DC progenitors reveals early lineage priming at the common DC progenitor stage in the bone marrow. Nature immunology 1-13.

Segura, E., Touzot, M., Bohineust, A., Cappuccio, A., Chiocchia, G., Hosmalin, A., Dalod, M., Soumelis, V., Amigorena, S., 2013. Human inflammatory dendritic cells induce Th17 cell differentiation. Immunity 38, 336-348.

Stellato, C., Collins, P., Ponath, P.D., Soler, D., Newman, W., La Rosa, G., Li, H., White, J., Schwiebert, L.M., Bickel, C., Liu, M., Bochner, B.S., Williams, T., Schleimer, R.P., 1997. Production of the novel C-C chemokine MCP-4 by airway cells and comparison of its biological activity to other C-C chemokines. Journal of Clinical Investigation 99, 926936.

Subramanian, A., Tamayo, P., Mootha, V.K., Mukherjee, S., Ebert, B.L., Gillette, M.A., Paulovich, A., Pomeroy, S.L., Golub, T.R., Lander, E.S., Mesirov, J.P., 2005. Gene set enrichment analysis: a knowledge-based approach for interpreting genome-wide expression profiles. Proceedings of the National Academy of Sciences of the United States of America 102, 15545-15550.

Széles, L., Keresztes, G., Töröcsik, D., Balajthy, Z., Krenács, L., Póliska, S., Steinmeyer, A., Zuegel, U., Pruenster, M., Rot, A., Nagy, L., 2009. 1,25-dihydroxyvitamin D3 is an autonomous regulator of the transcriptional changes leading to a tolerogenic dendritic cell phenotype. Journal of immunology 182, 2074-2083.

Toujas, L., Delcros, J.G., Diez, E., Gervois, N., Semana, G., Corradin, G., Jotereau, F., 1997. Human monocyte-derived macrophages and dendritic cells are comparably effective in vitro in presenting HLA class I-restricted exogenous peptides. Immunology 91, 635-642.

Tumbarello, D.A., Kendrick-Jones, J., Buss, F., 2013. Myosin VI and its cargo adaptors linking endocytosis and autophagy. Journal of Cell Science 126, 2561-2570.

Varol, C., Mildner, A., Jung, S., 2015. Macrophages: development and tissue specialization. Annual review of immunology 33, 643-675.

Wu, L., D'Amico, A., Winkel, K.D., Suter, M., Lo, D., Shortman, K., 1998. RelB is essential for the development of myeloid-related CD8alpha- dendritic cells but not of lymphoidrelated CD8alpha+ dendritic cells. Immunity 9, 839-847.

Xu, A.M., Aalipour, A., Leal-Ortiz, S., Mekhdjian, A.H., Xie, X., Dunn, A.R., Garner, C.C., Melosh, N.A., 2014. Quantification of nanowire penetration into living cells. Nat Commun 5, 3613.

Xue, J., Schmidt, S.V., Sander, J., Draffehn, A., Krebs, W., Quester, I., De Nardo, D., Gohel, T.D., Emde, M., Schmidleithner, L., Ganesan, H., Nino-Castro, A., Mallmann, M.R., Labzin, L., Theis, H., Kraut, M., Beyer, M., Latz, E., Freeman, T.C., Ulas, T., Schultze, J.L., 2014. Transcriptome-Based Network Analysis Reveals a Spectrum Model of Human Macrophage Activation. Immunity 40, 274-288. 
Yu, P., Wang, Y., Chin, R.K., Martinez-Pomares, L., Gordon, S., Kosco-Vibois, M.H., Cyster, J., Fu, Y.X., 2002. B Cells Control the Migration of a Subset of Dendritic Cells into B Cell Follicles Via CXC Chemokine Ligand 13 in a Lymphotoxin-Dependent Fashion. Journal of immunology 168, 5117-5123. 
Figure 1

A

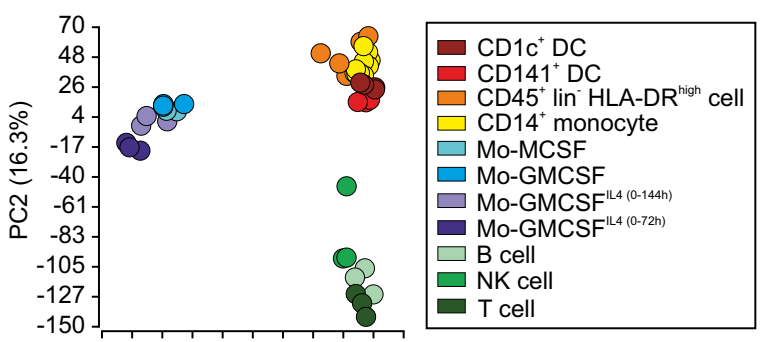

它 순

PC1 $(20.7 \%)$

D

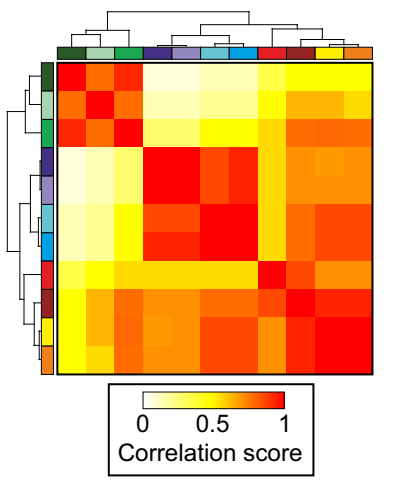

$\mathbf{F}$
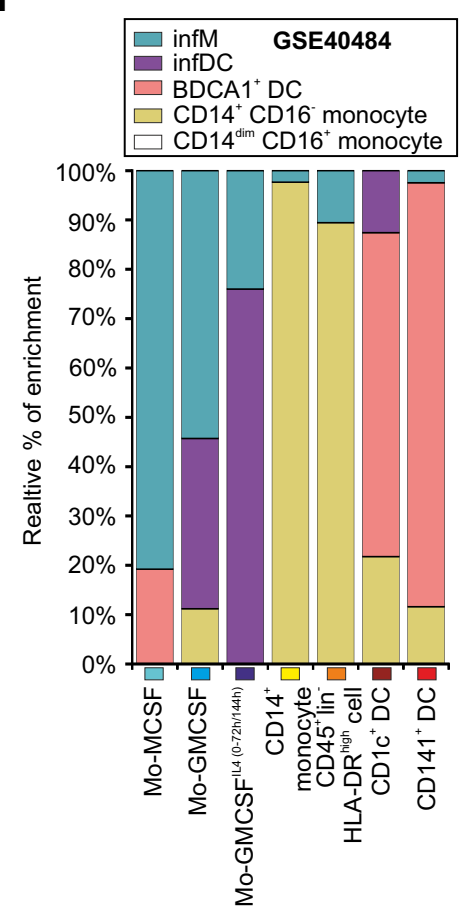

I
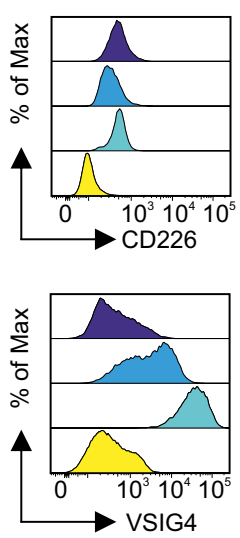

B

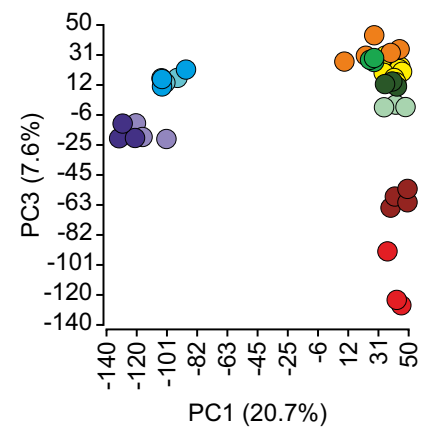

E

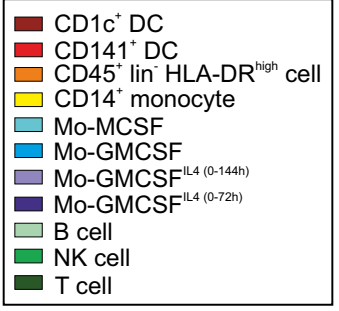

$\square$ T cell
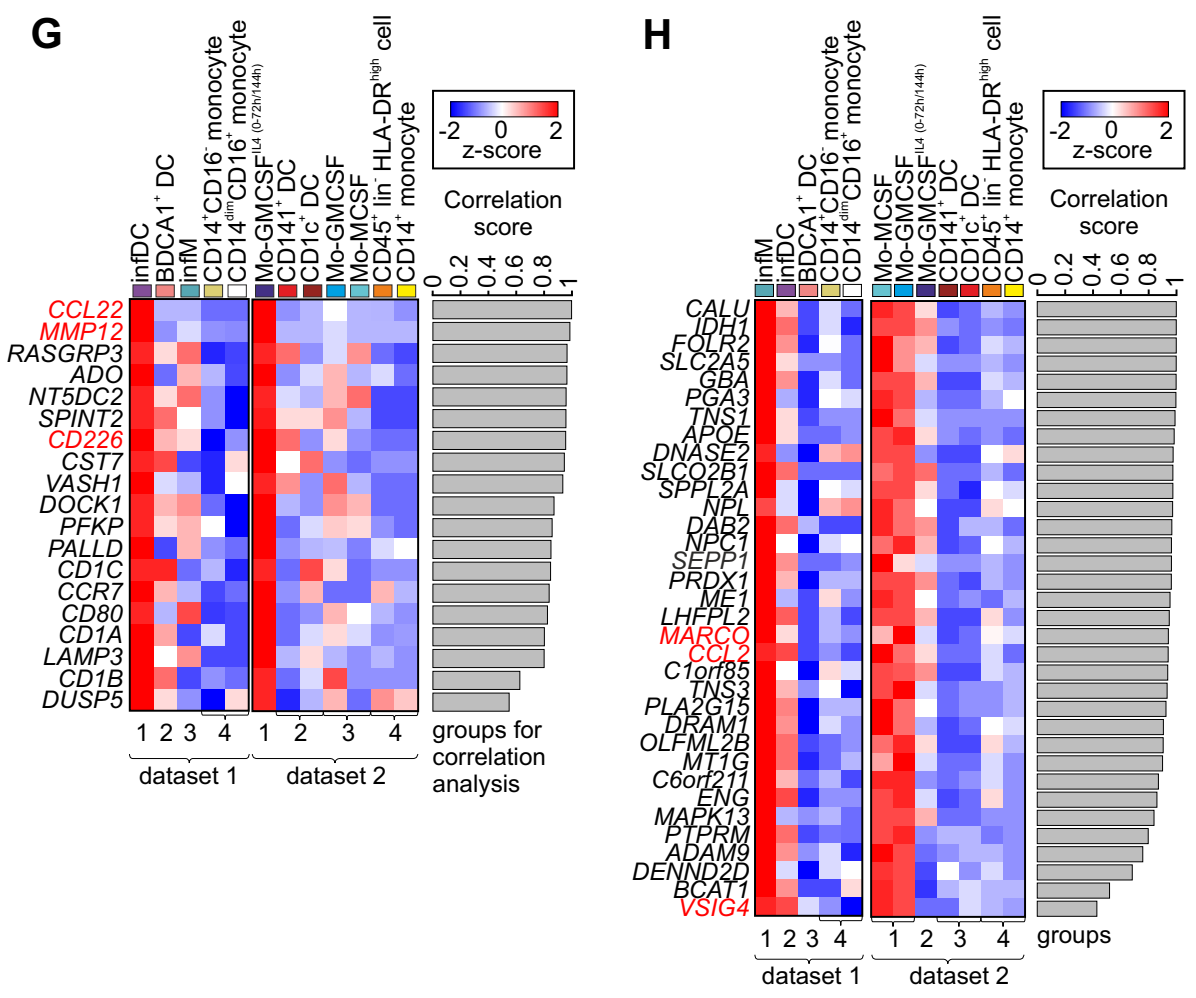

K

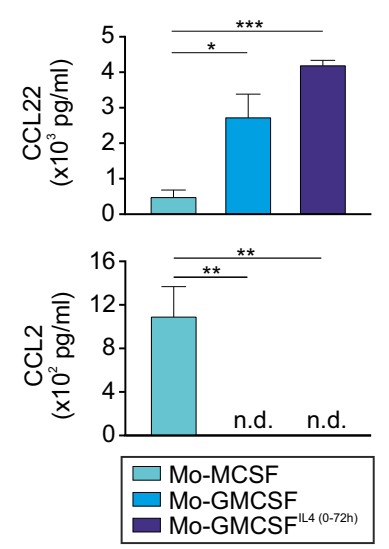

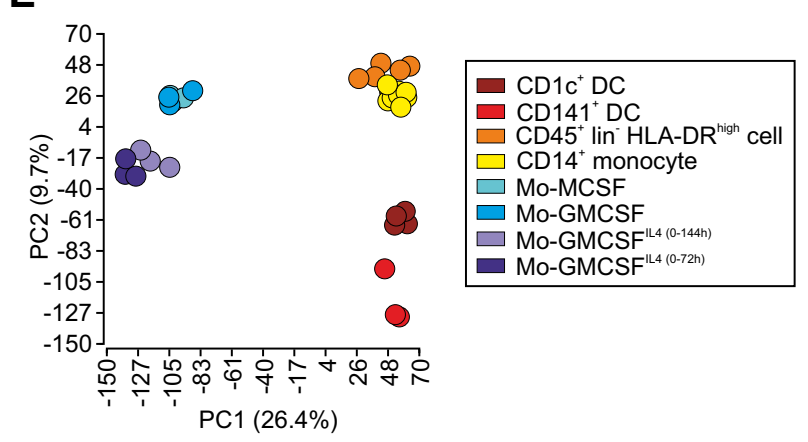

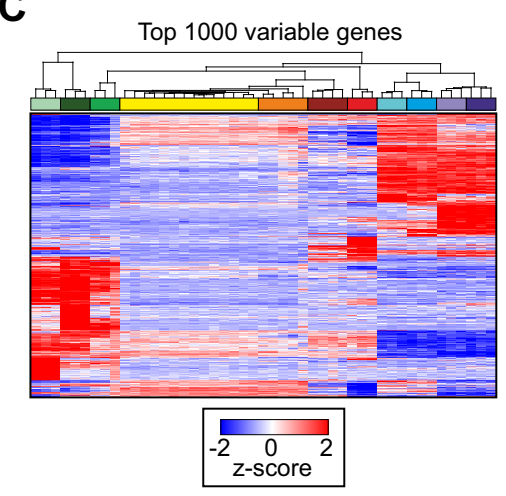

\section{C}

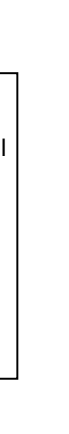




\section{Figure 2}

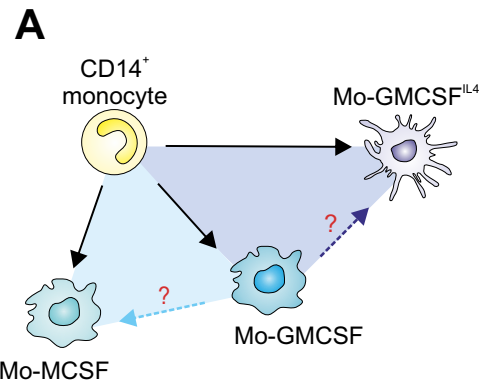

D

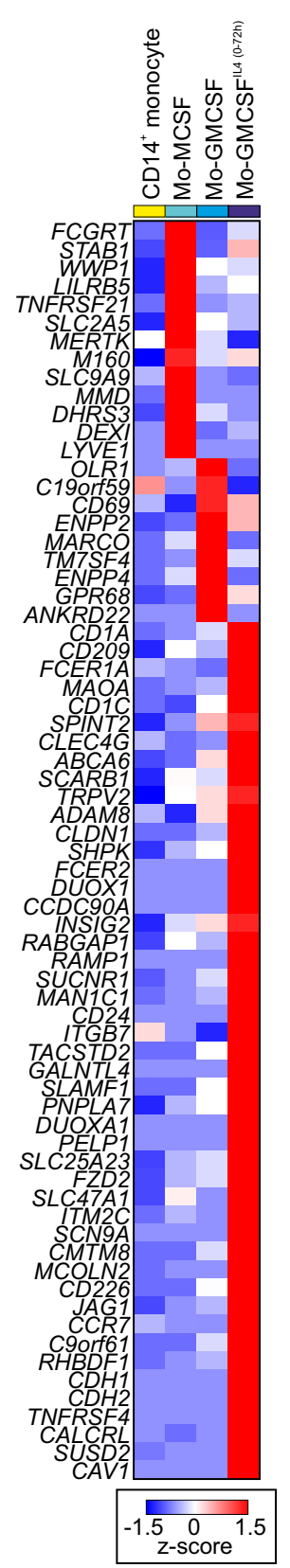

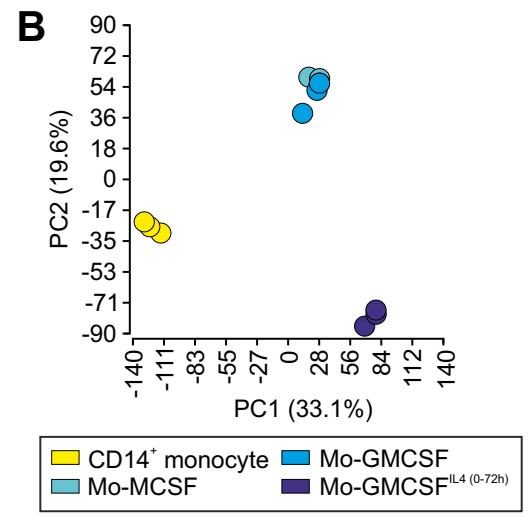

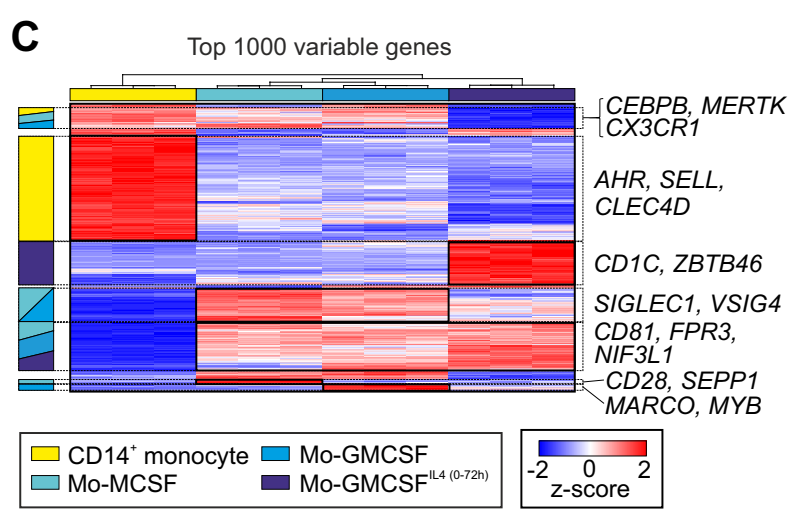

$\mathbf{F}$
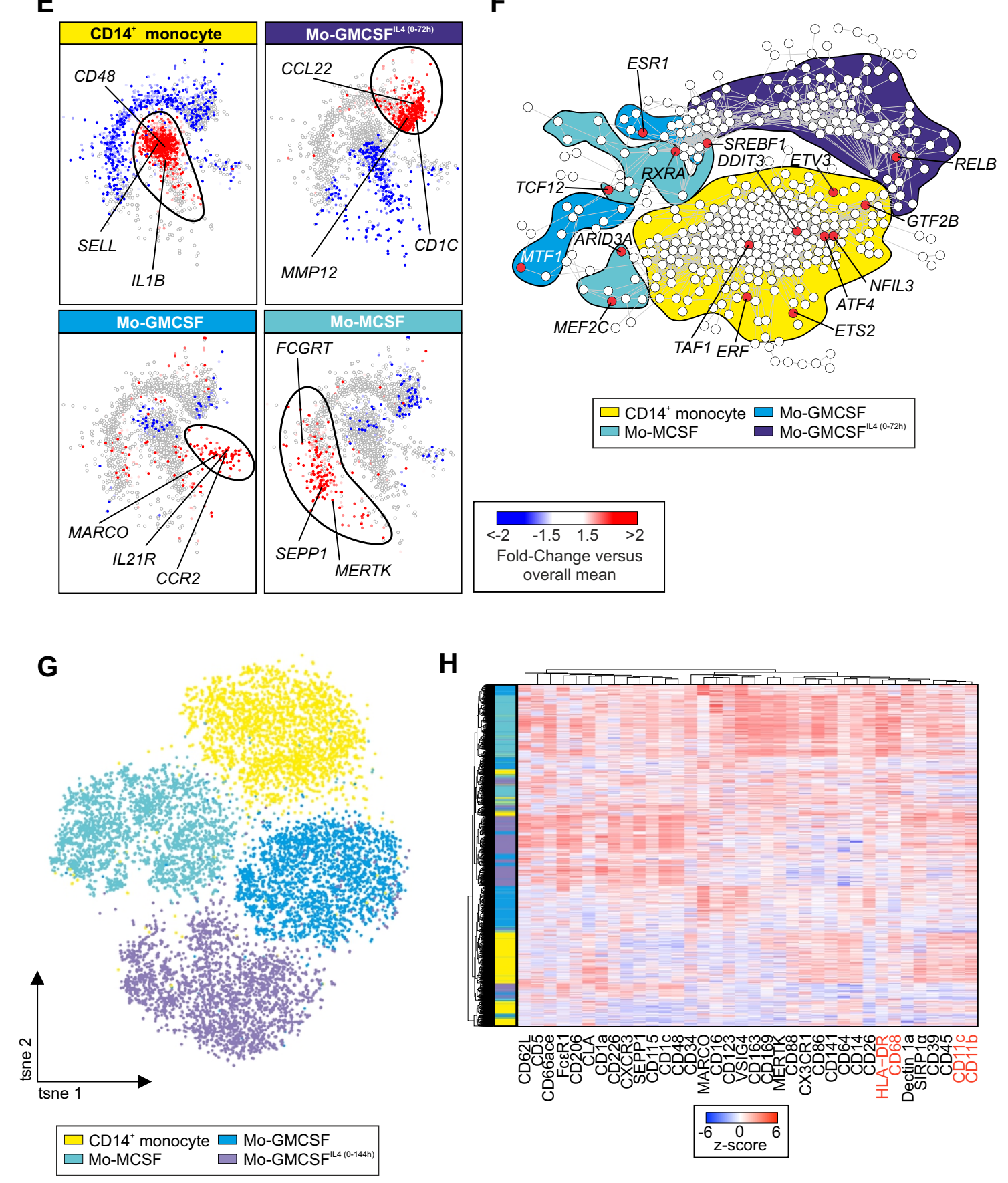

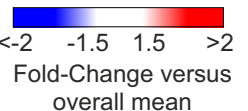

overall mean
H

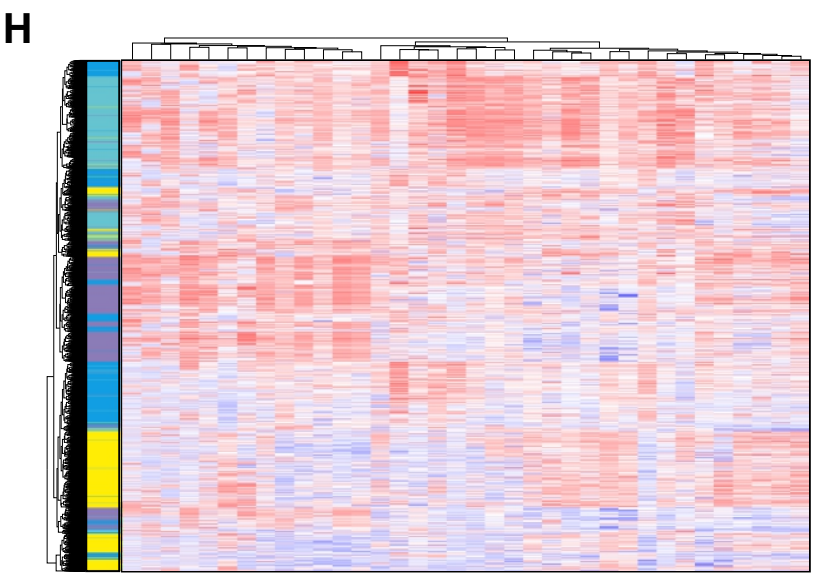

సొนల

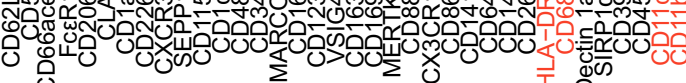
$-6 \underset{ }{20} 6$ 


\section{Figure 3}

A

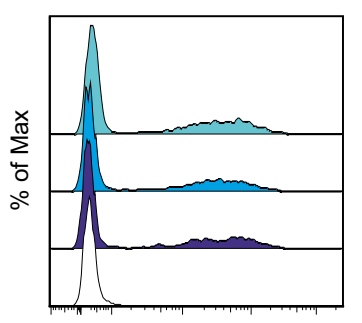

GFP $^{+}$yeast

$\square$ Mo-MCSF $\square$ Mo-GMCSF Mo-GMCSF $^{(1-72 h)}$
$\square$ control

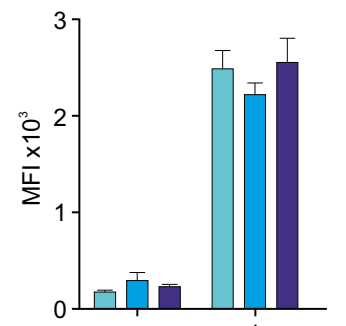

GFP $^{+}$yeast
B

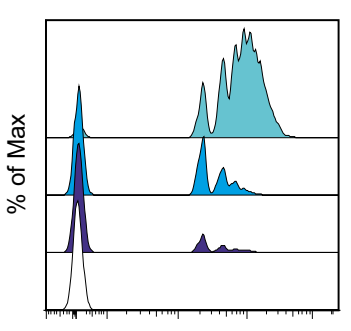

YG beads

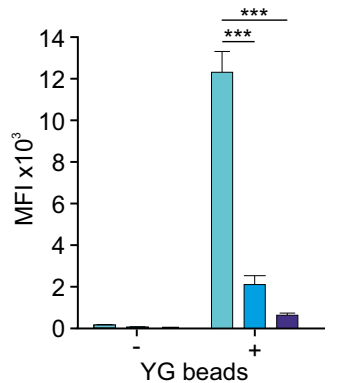

$\square$ Mo-MCSF $\square$ Mo-GMCSF ${ }^{1 ! 4(0-72 h)}$ $\square$ Mo-GMCSF $\square$ control

C
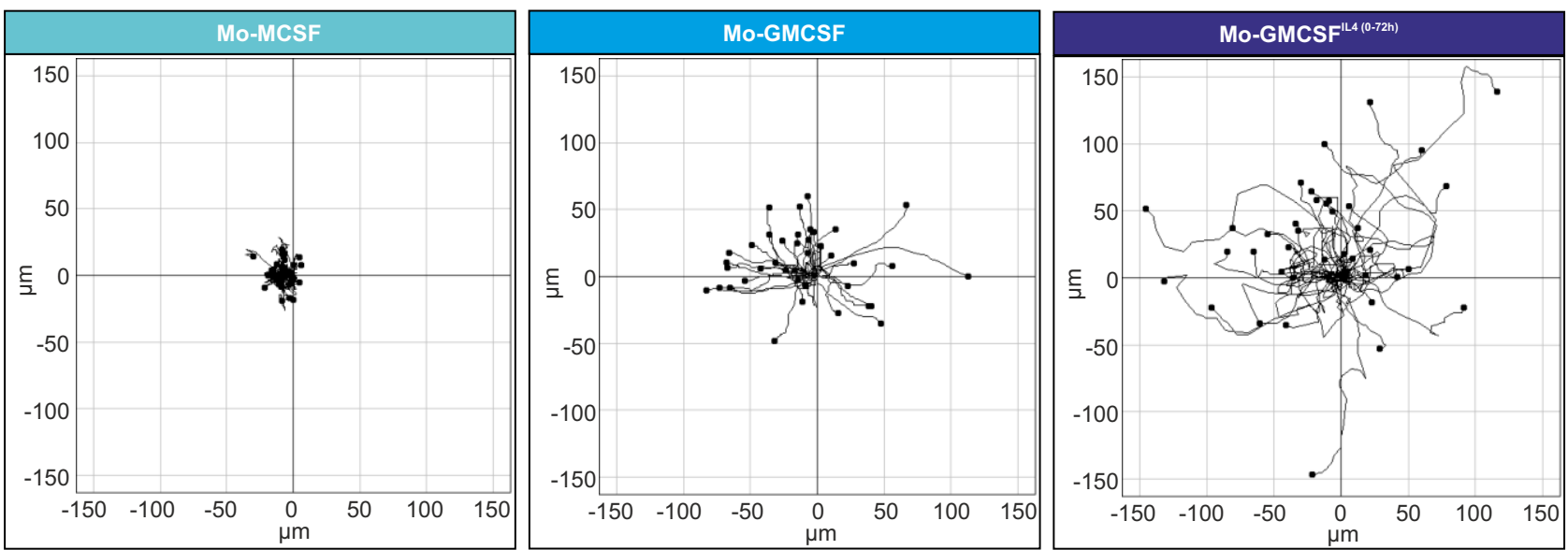

D

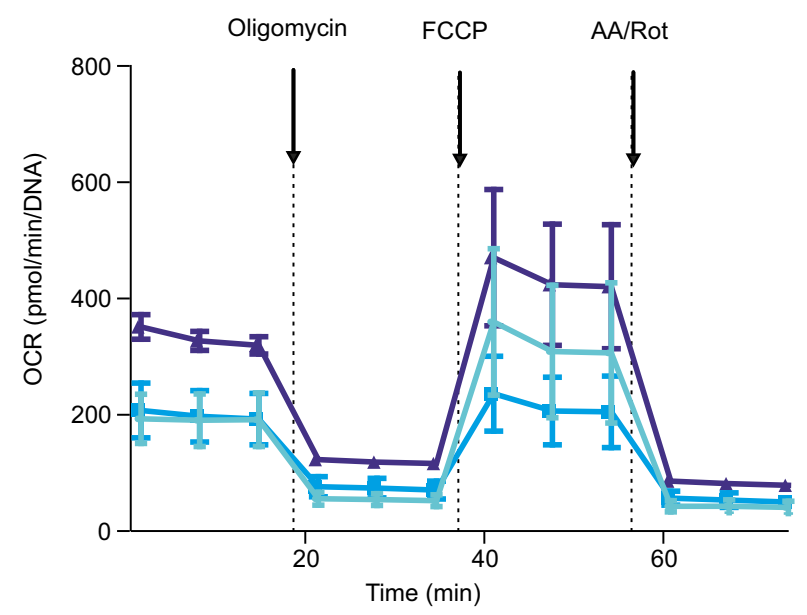

$\mathbf{E}$

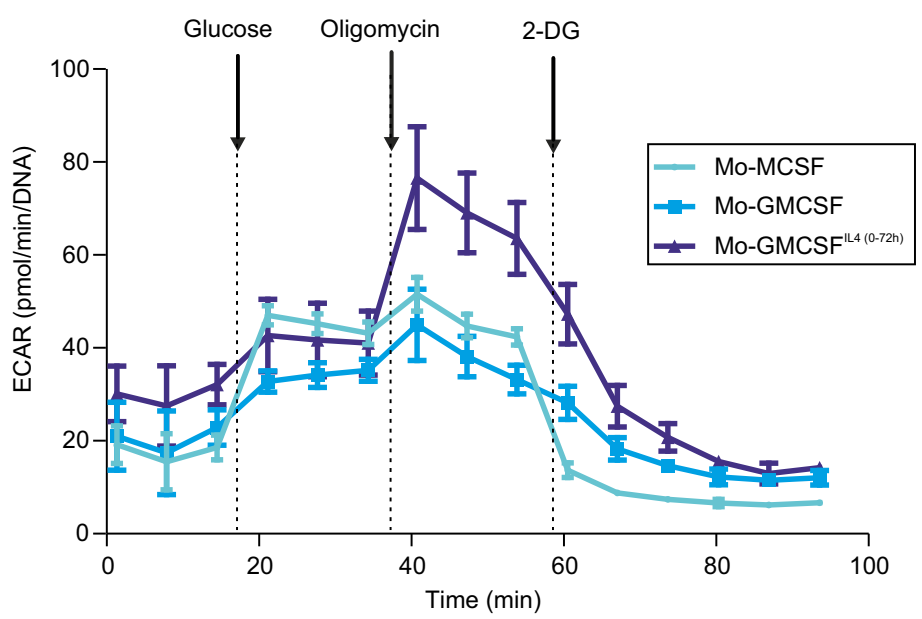

$\mathbf{F}$

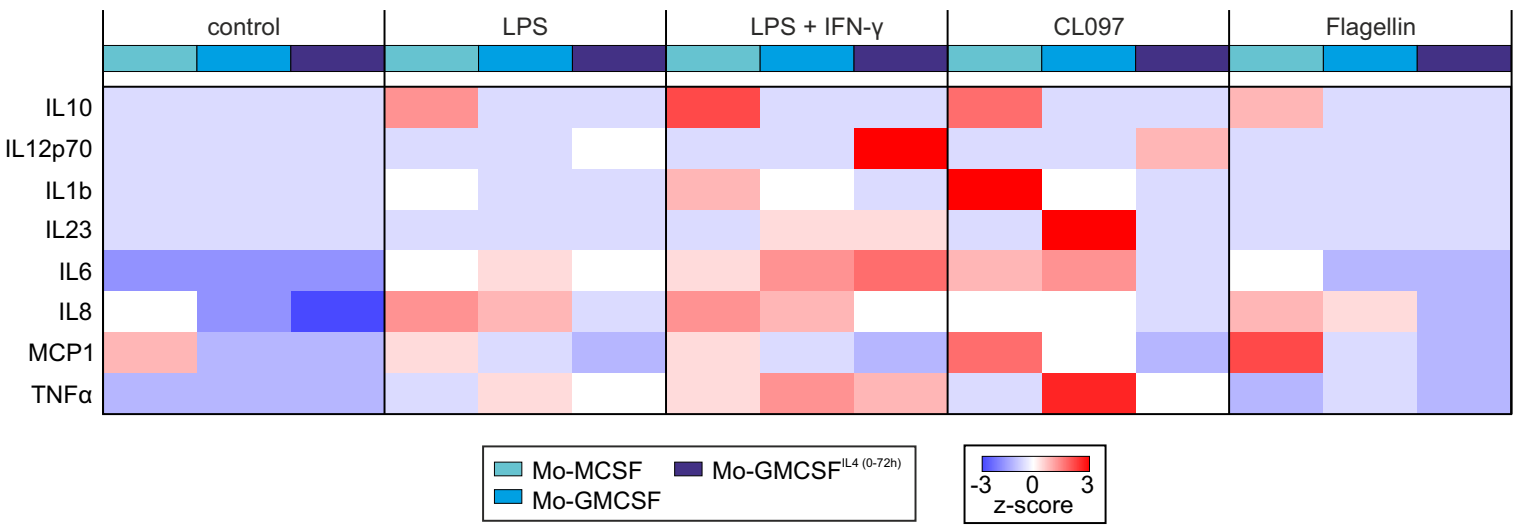


bioRxiv preprint doi: https://doi.org/10.1101/204180; this version posted October 16,2017 . The copyright holder for this preprint (which was not certified by peer review) is the author/funder. All rights reserved. No reuse allowed without permission.

\section{Figure 4}

A

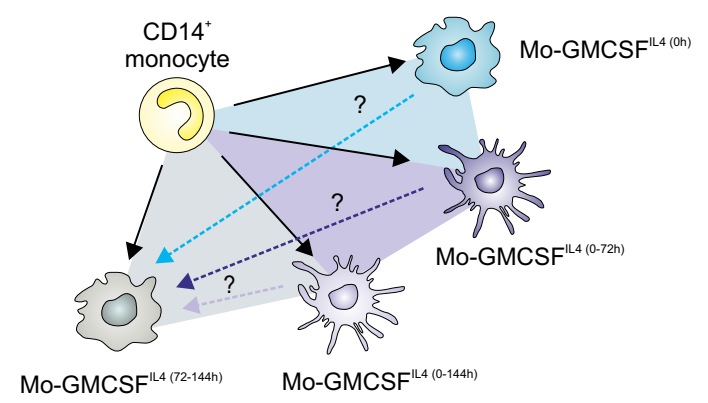

C

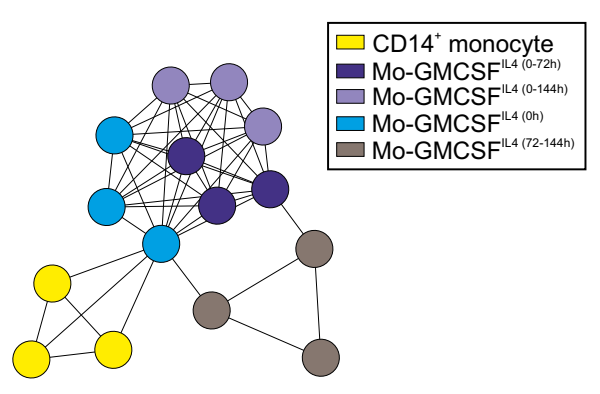

$\mathbf{E}$

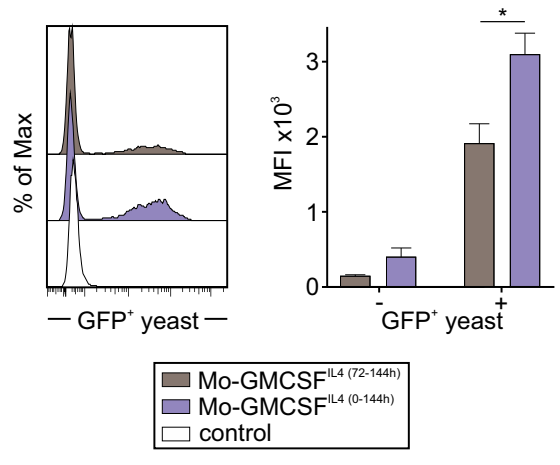

\section{B}

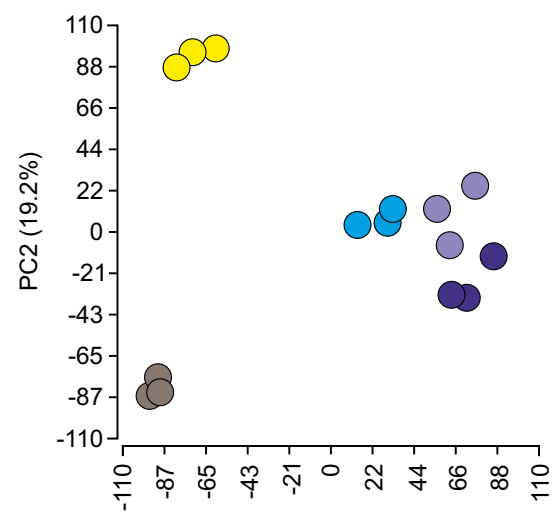

PC1 (27.7\%)

\begin{tabular}{|c|}
\hline 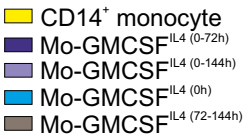 \\
\hline
\end{tabular}

D

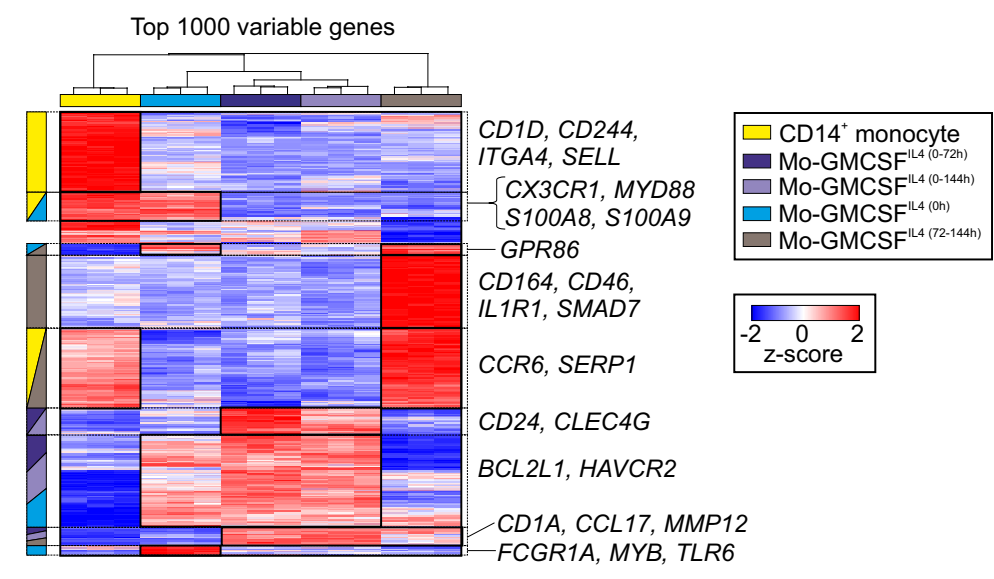

$\mathbf{F}$

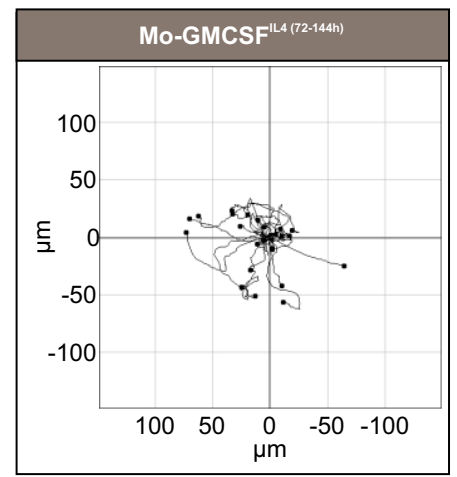




\section{Figure 5}

A

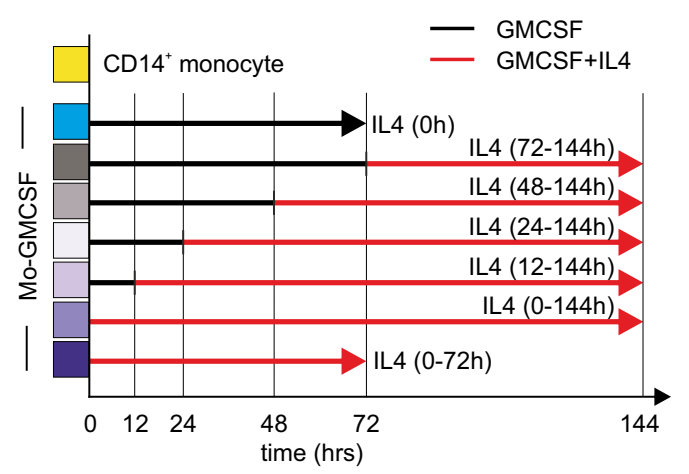

B

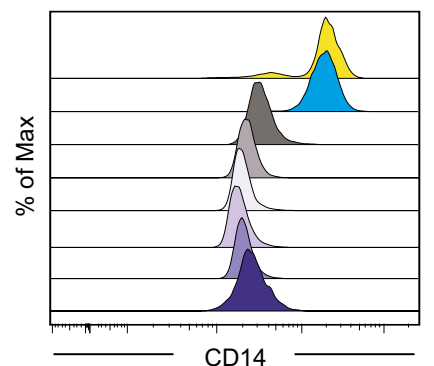

C

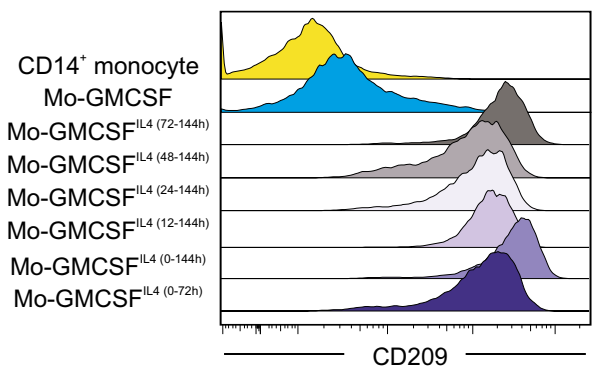

D

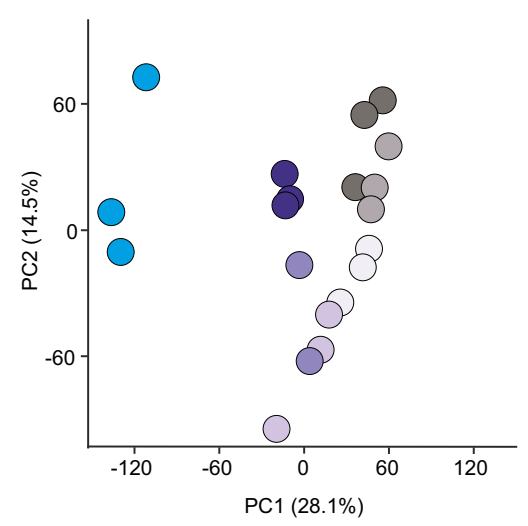

$\mathbf{E}$

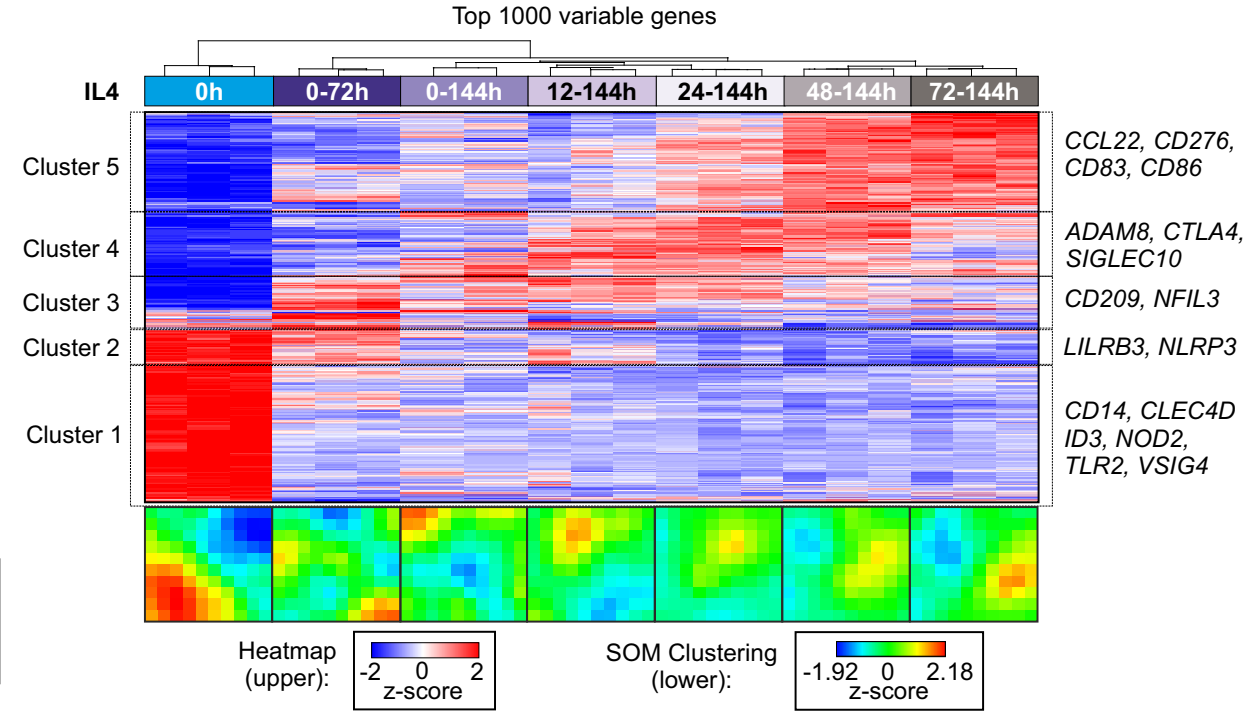

$\mathbf{F}$

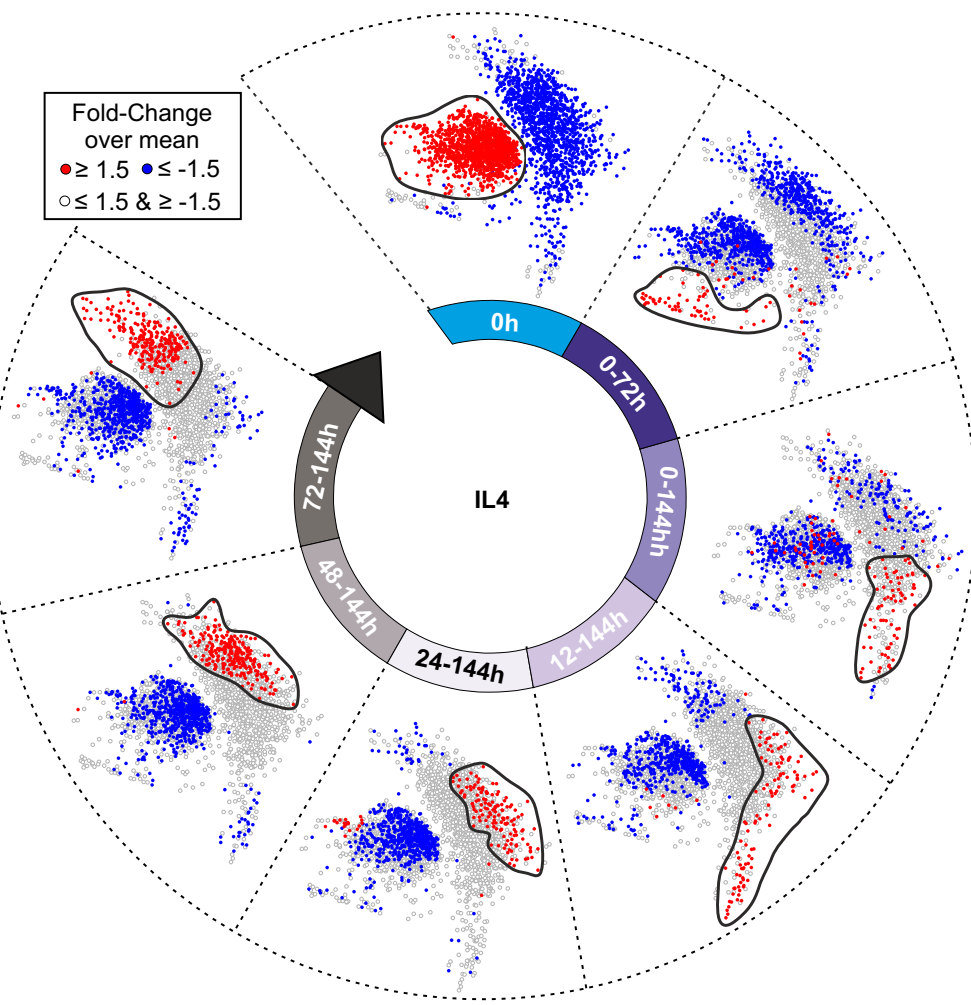

G

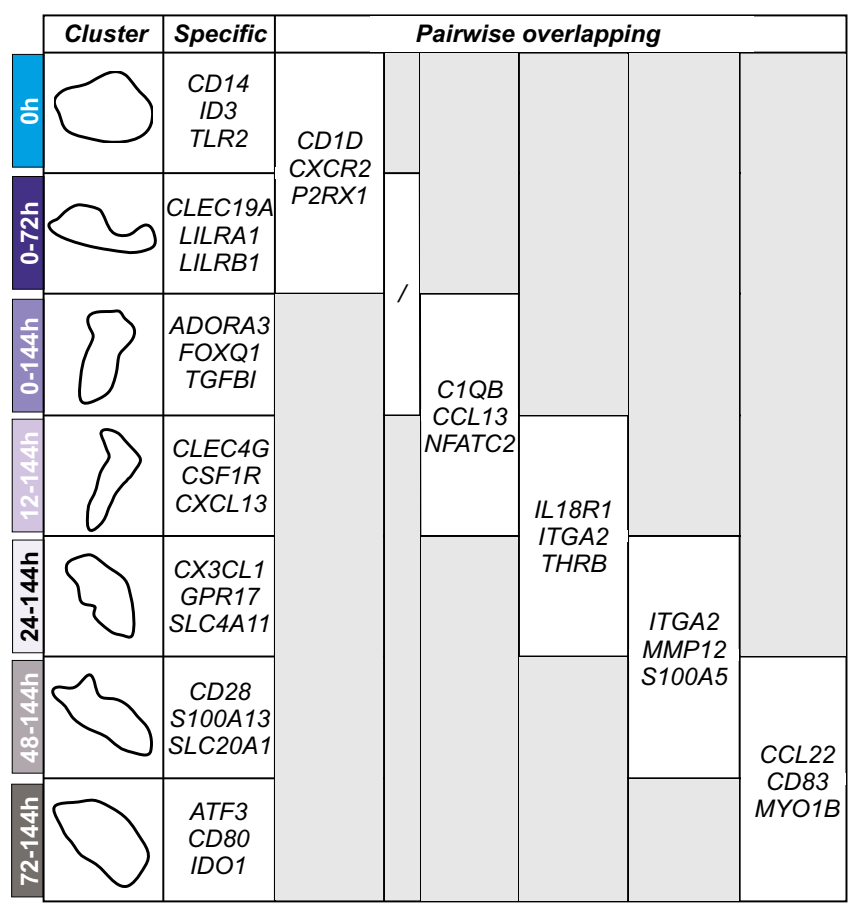




\section{Figure 6}

A

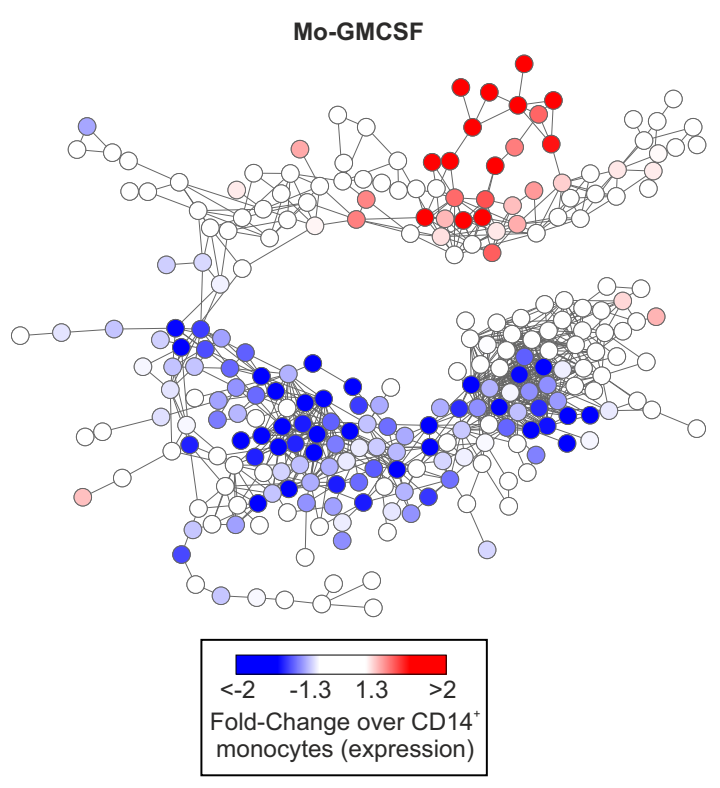

C

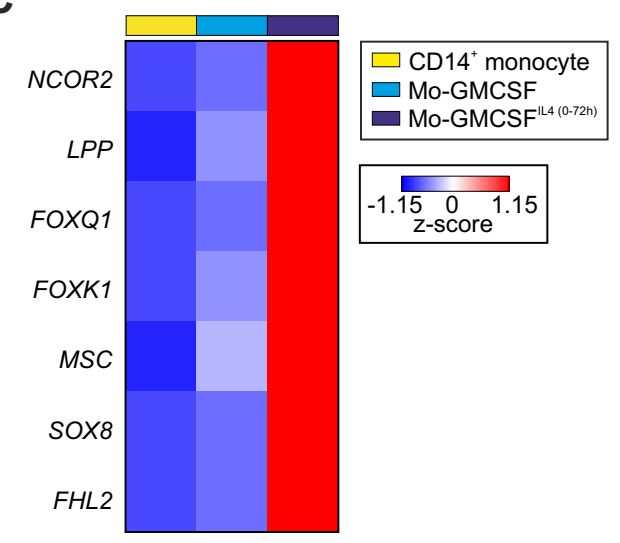

E

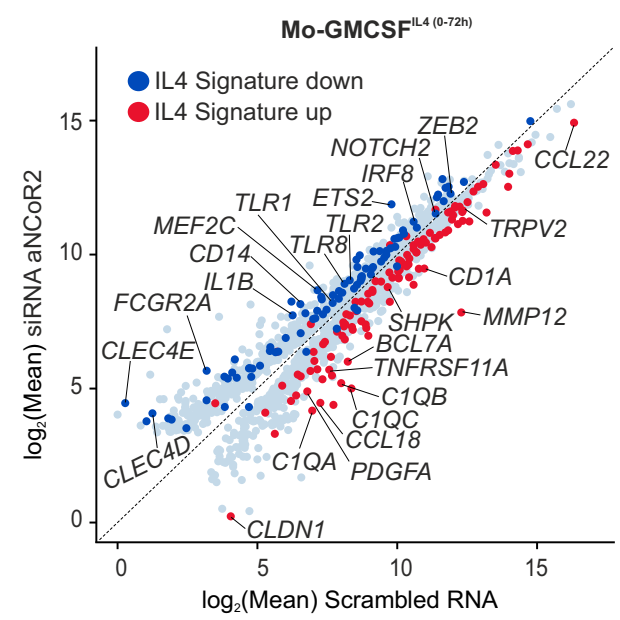

B

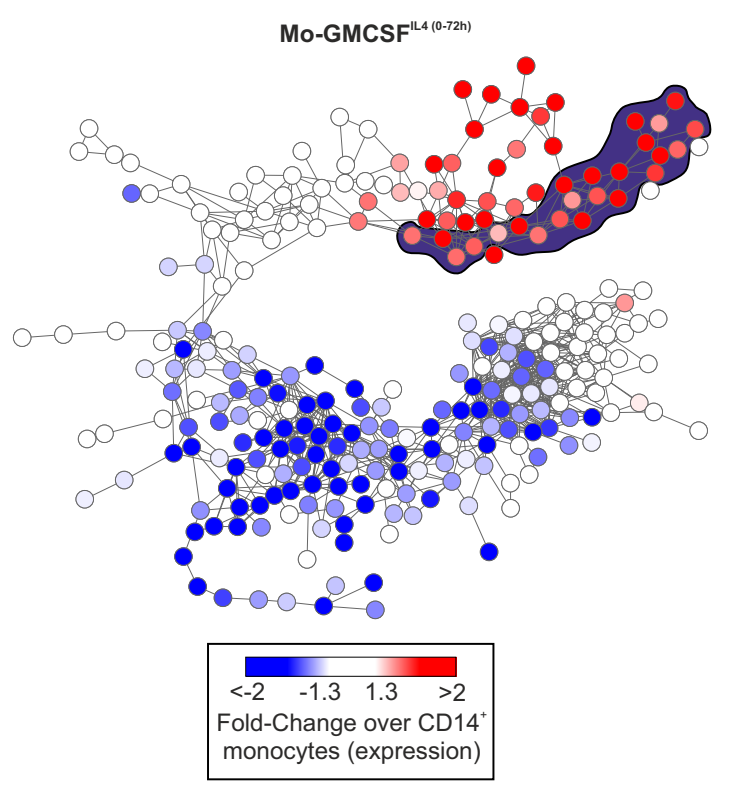

D

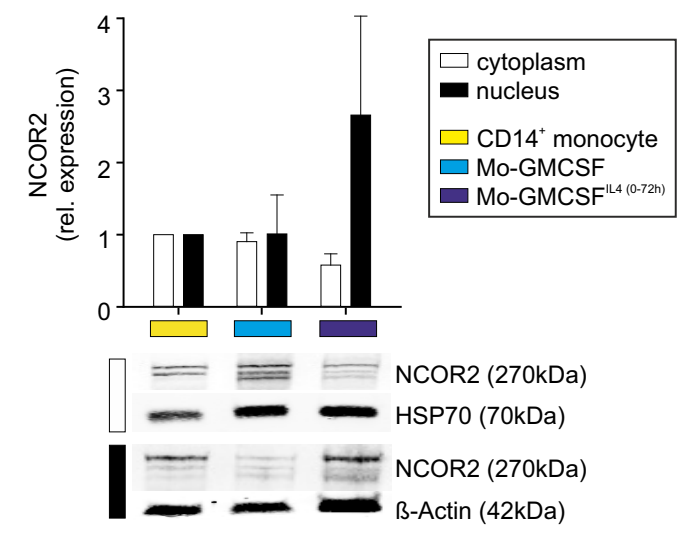




\section{Figure 7}

A

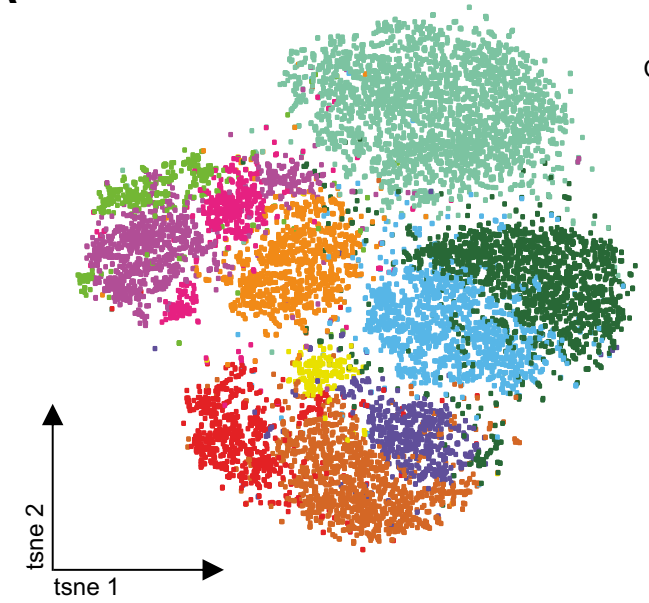

C

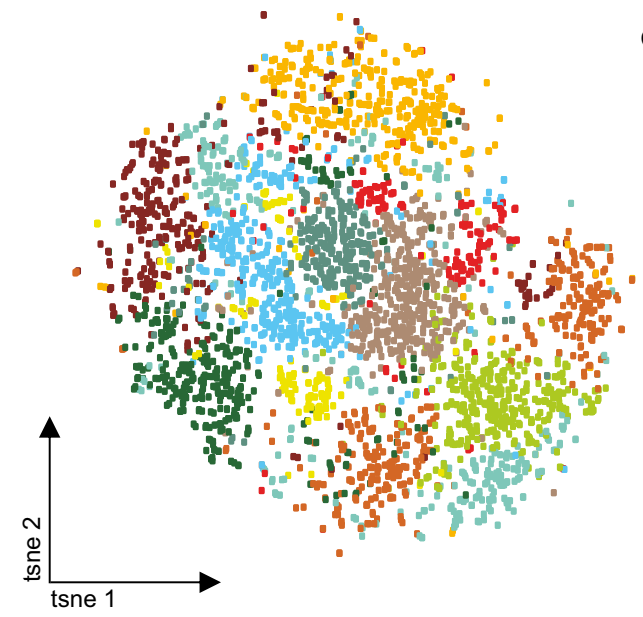

B
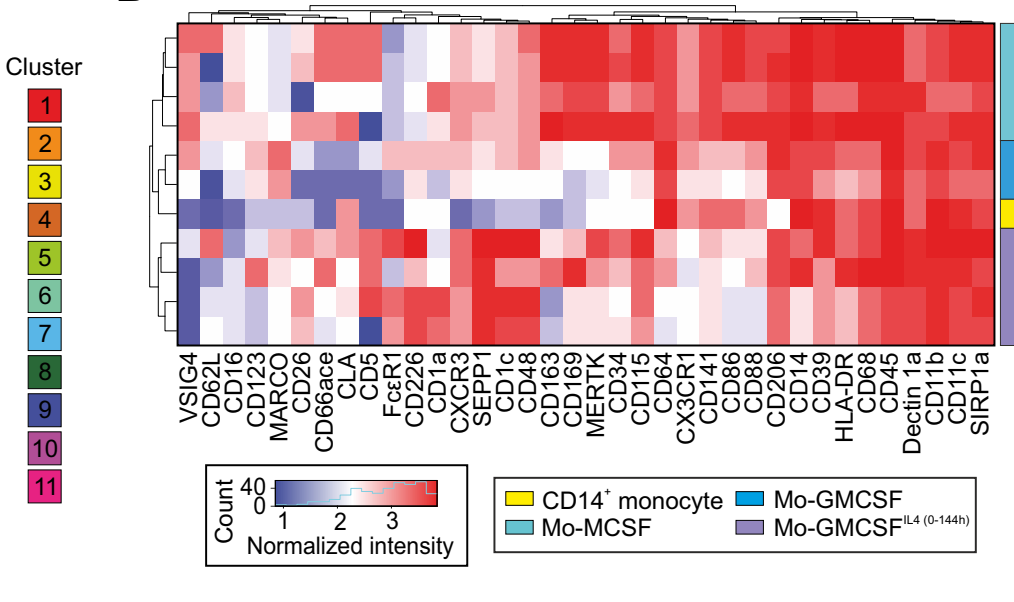

D

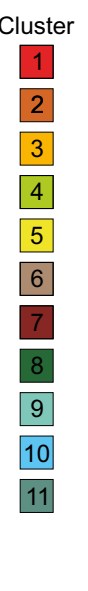

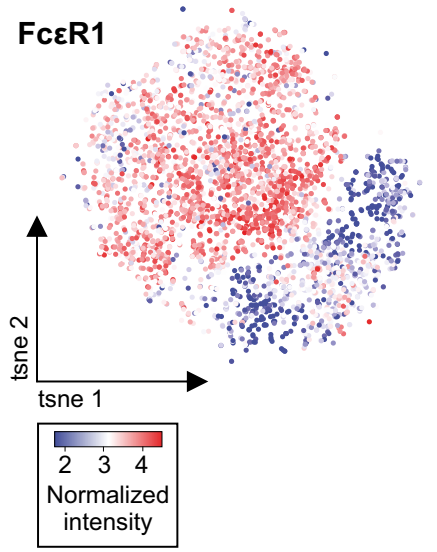

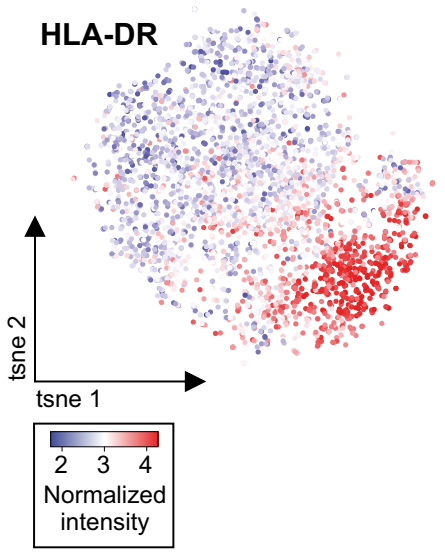

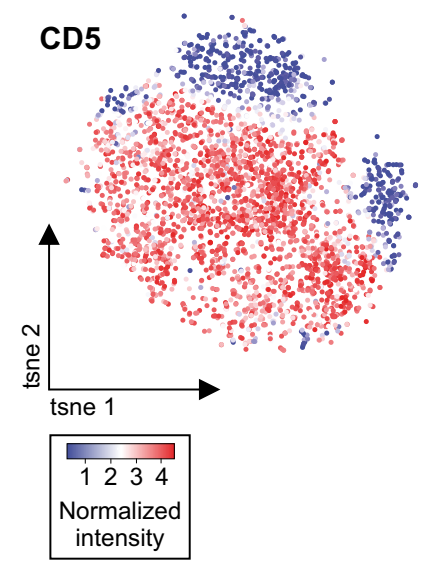
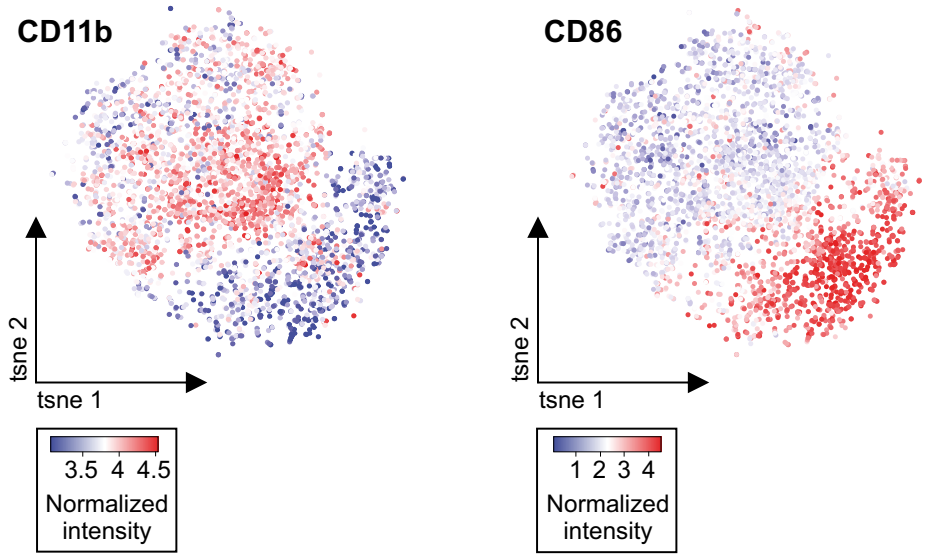
bioRxiv preprint doi: https://doi.org/10.1101/204180; this version posted October 16, 2017. The copyright holder for this preprint (which was not certified by peer review) is the author/funder. All rights reserved. No reuse allowed without permission.

\section{Supplementary figure 1}

A

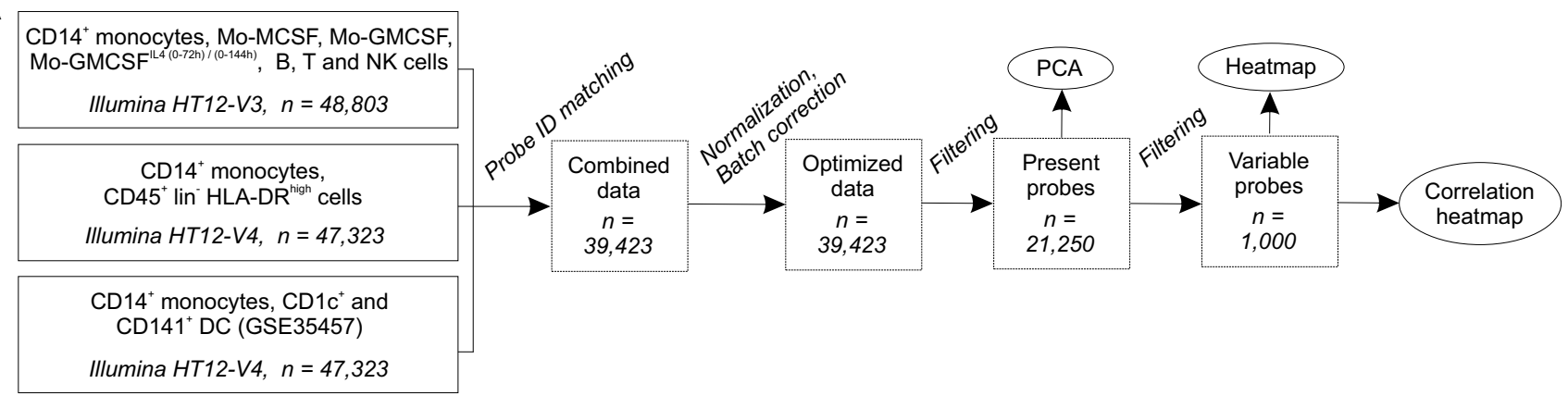

B
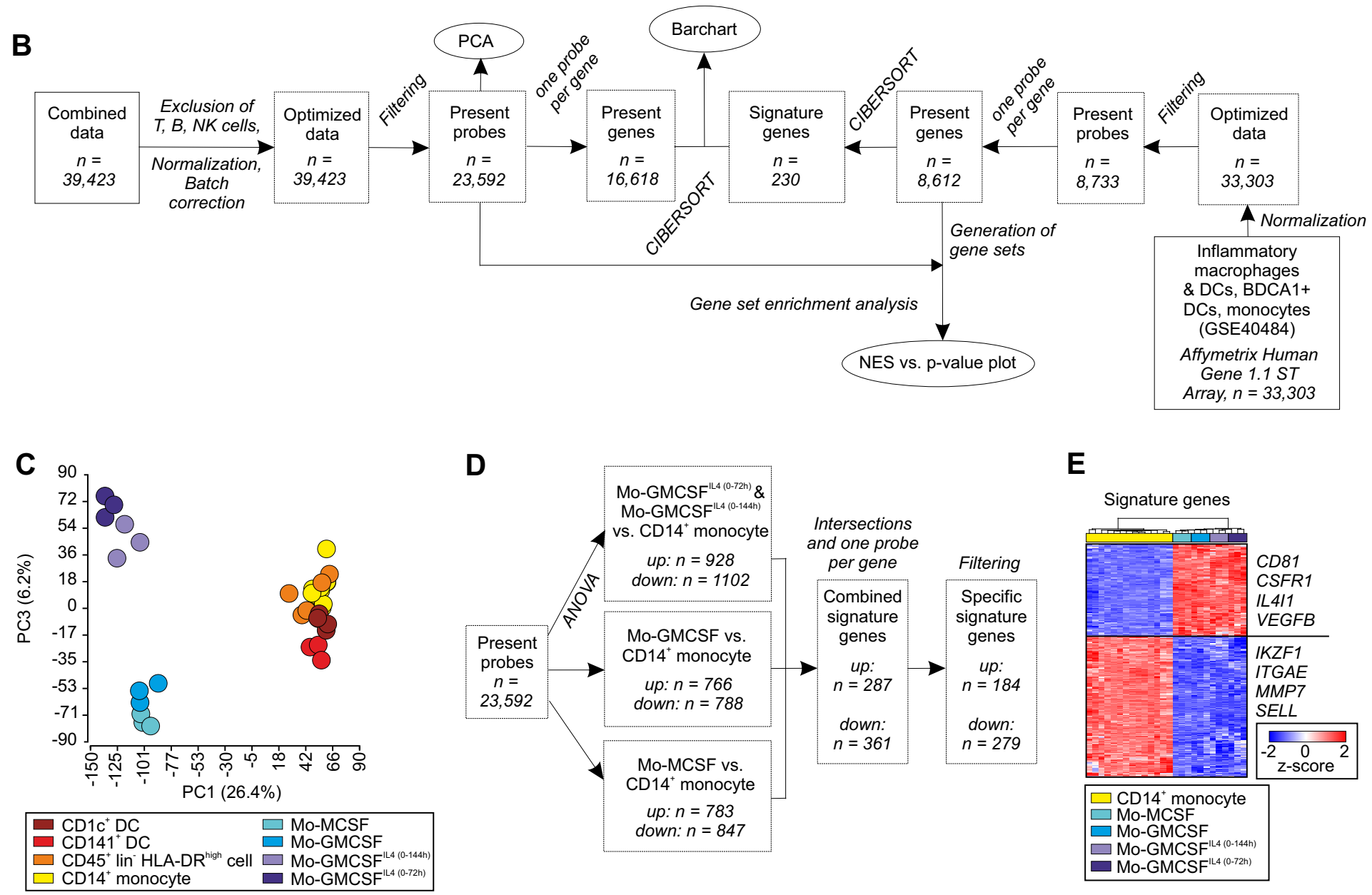

E

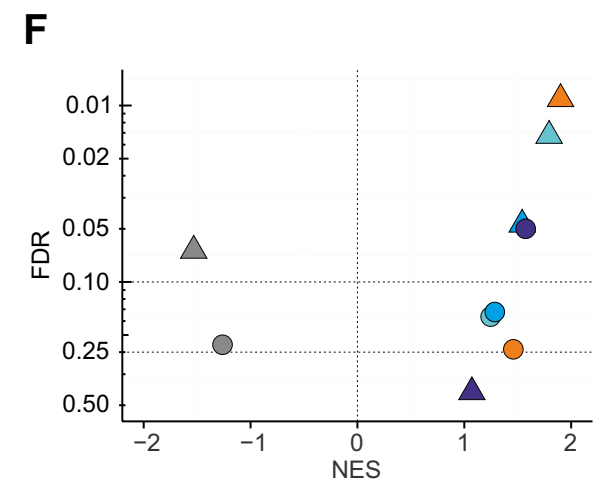

G
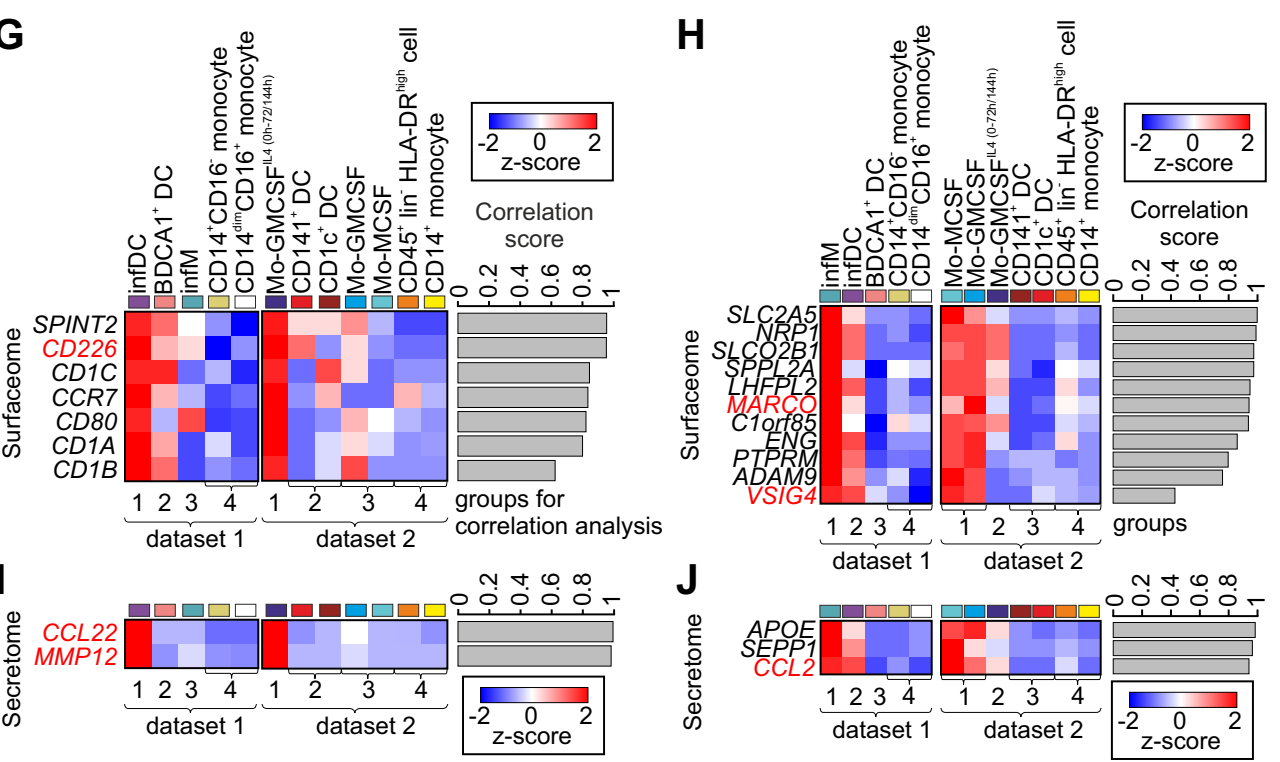

$\mathrm{CD}_{141^{+} \mathrm{DC} \text { vs CD1C }}^{+} \mathrm{DC}$

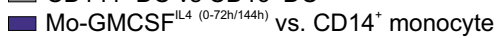

$\square$ Mo-GMCSF vs. CD14 ${ }^{+}$monocyte

$\square$ Mo-MCSF vs. CD14 monocyte

$\square \mathrm{CD}^{+} 5^{+}$lin HLA-DR ${ }^{\text {high }}$ vs. CD14 ${ }^{+}$monocyte

- infDC vs. CD14 monocyte

$\Delta$ infM vs. CD14 ${ }^{+}$monocyte

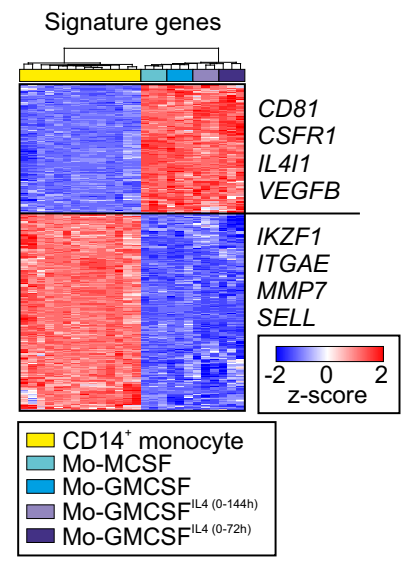




\section{Supplementary figure 2}

A

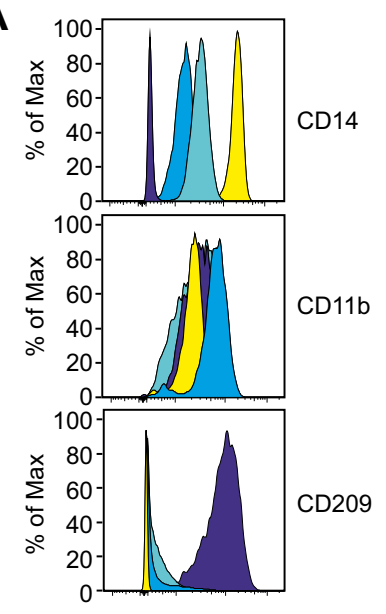

B

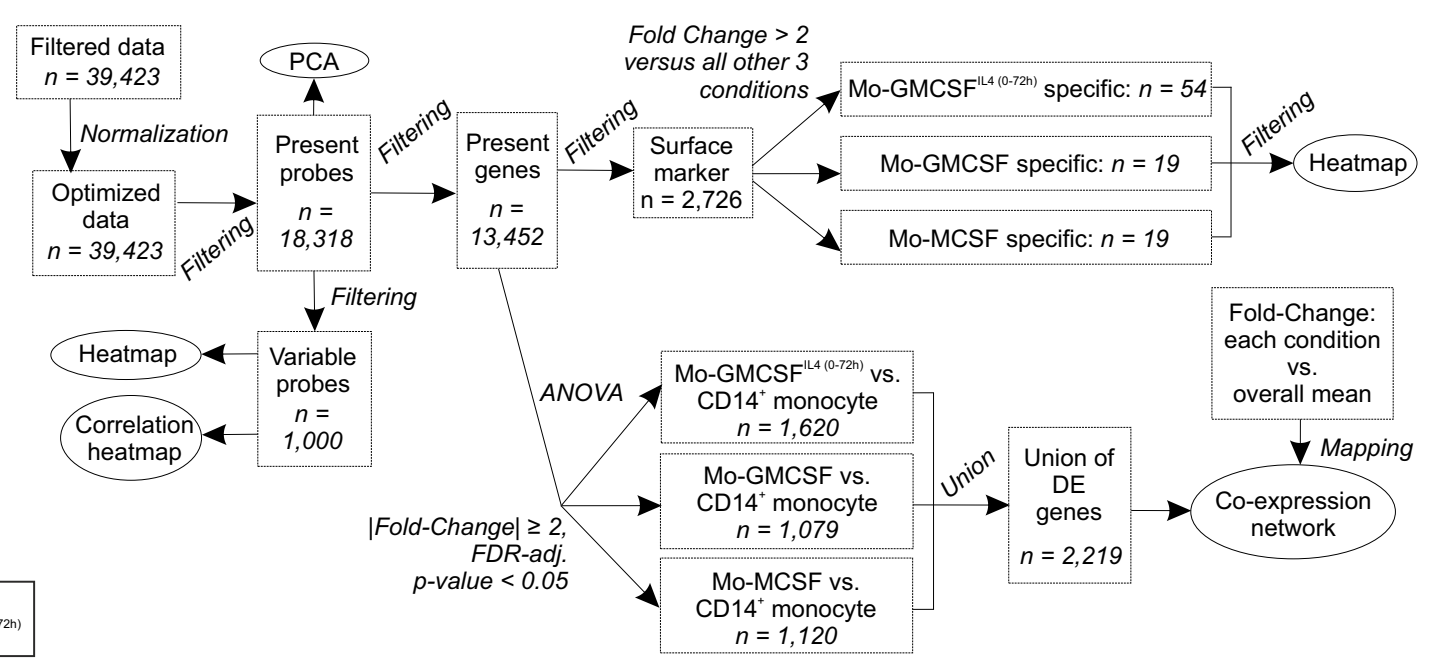

C

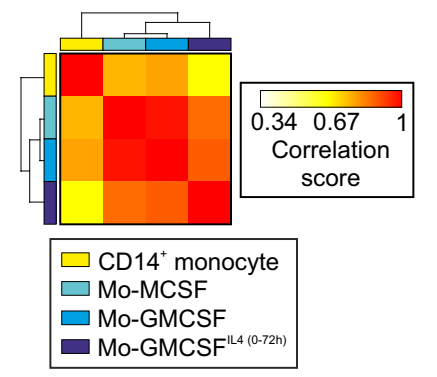

$\mathbf{E}$

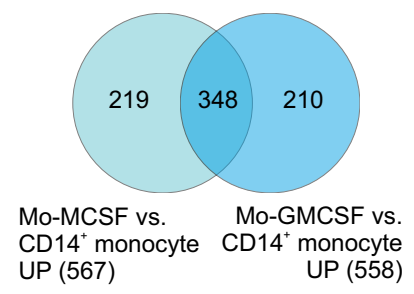

D

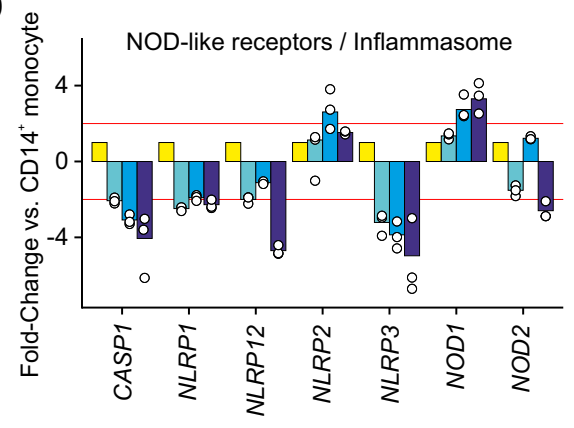

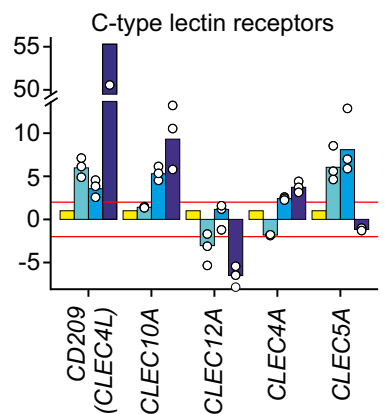

Toll-like receptors

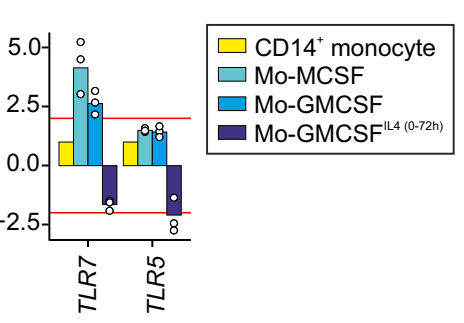

$\mathbf{F}$

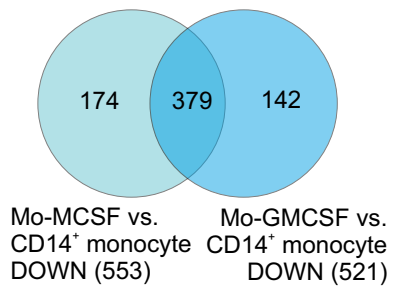

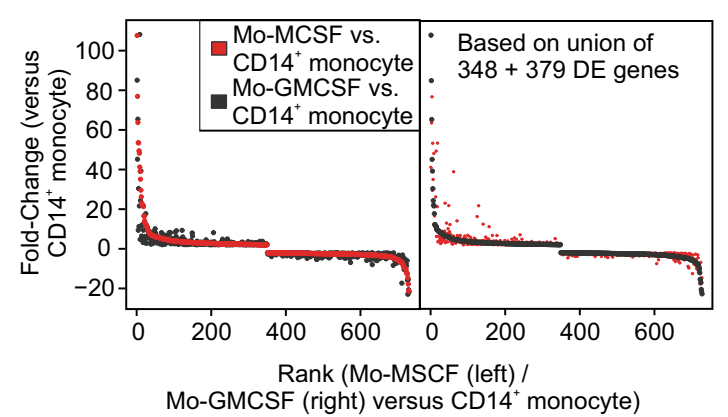

Mo-GMCSF (right) versus CD14 ${ }^{+}$monocyte)
G

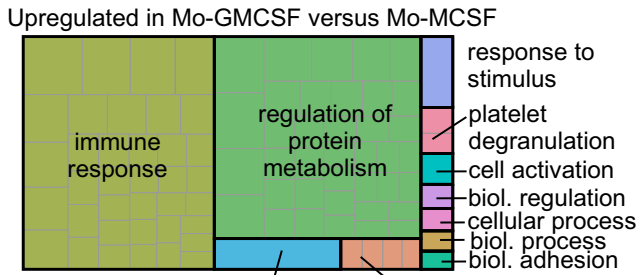

immune system process alcohol metabolism

Upregulated in Mo-MCSF versus Mo-GMCSF

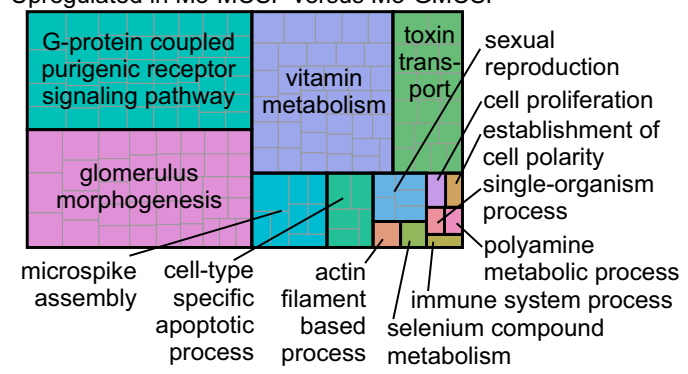

H

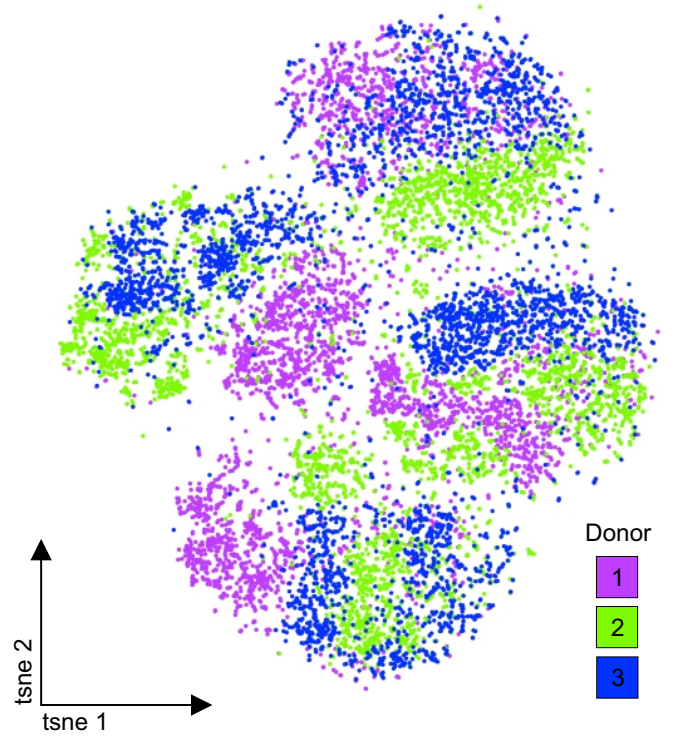




\section{Supplementary figure 3}

A

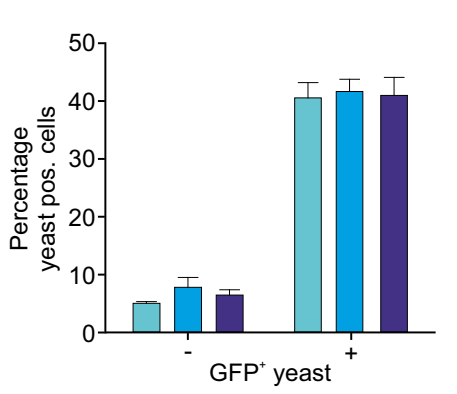

C

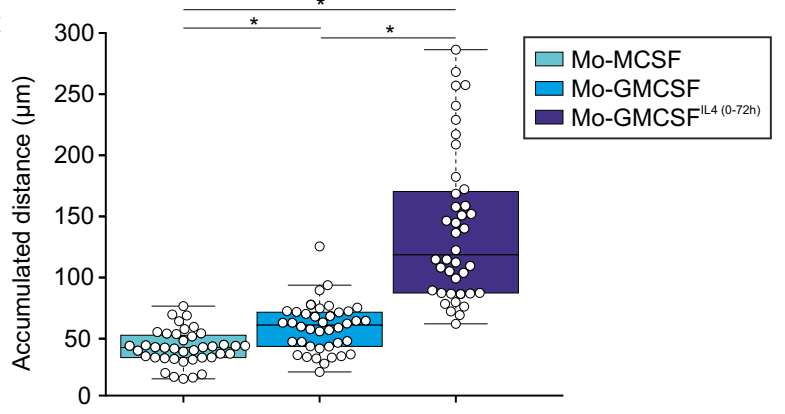

E

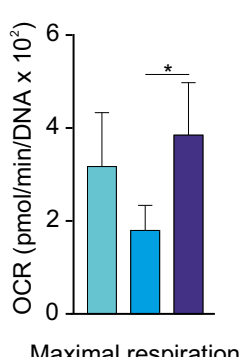

Maximal respiration

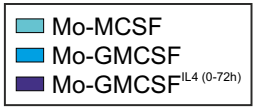

$\mathbf{F}$

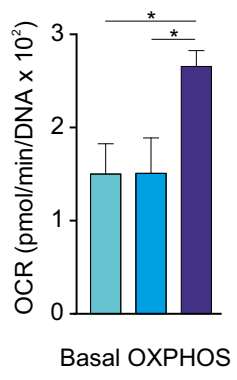

G

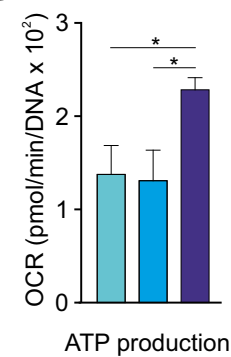

B

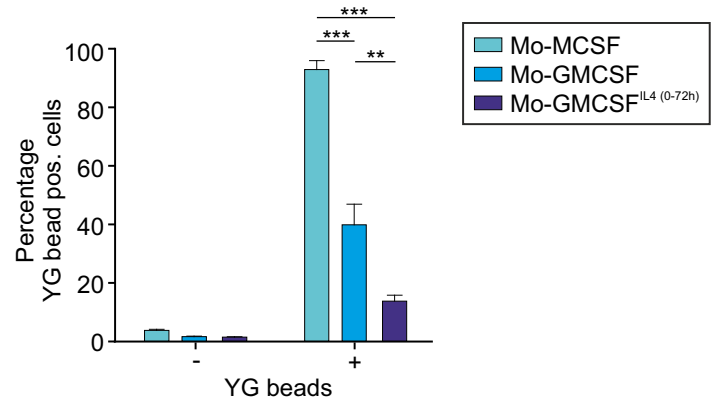

D

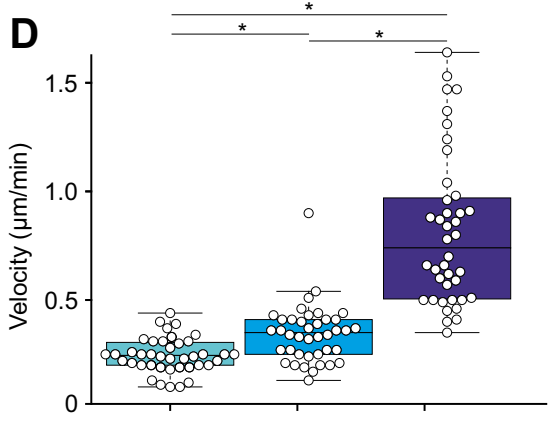

H

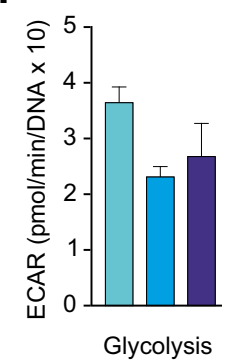

$\square$ Mo-MCSF

$\square$ Mo-GMCSF

Mo-GMCSF $^{1 L 4(0-72 \mathrm{~h})}$
J

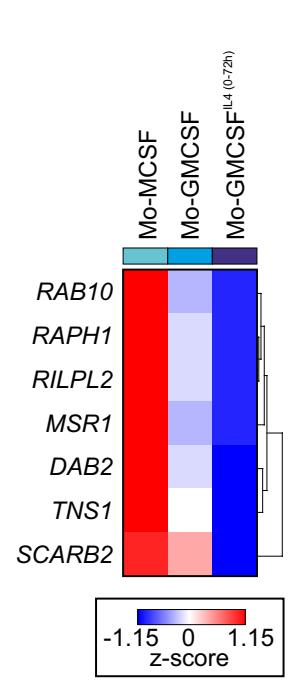

K

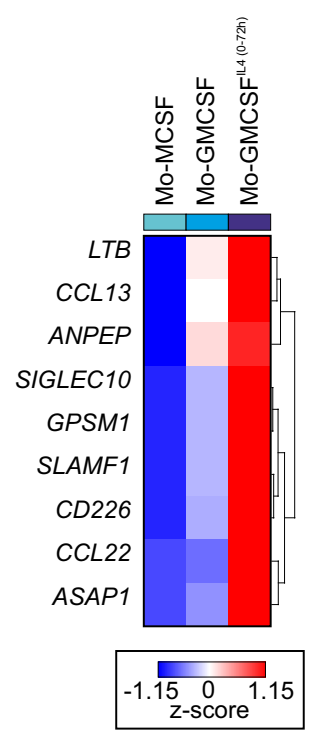

L

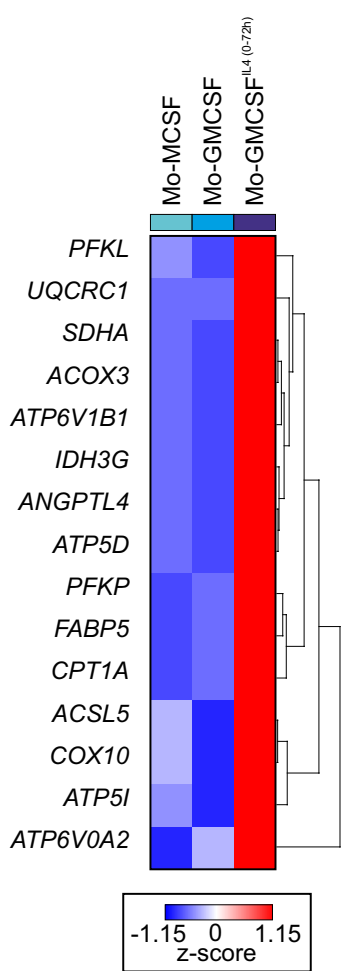




\section{Supplementary figure 4}

A
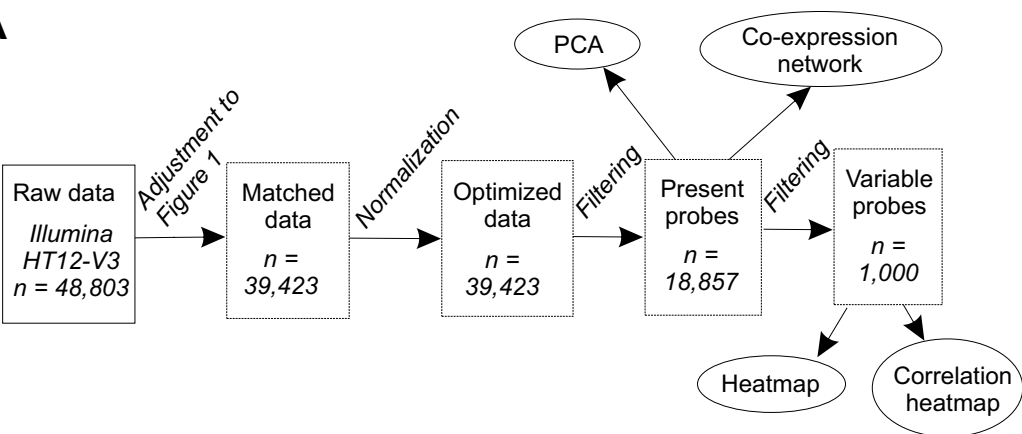

C
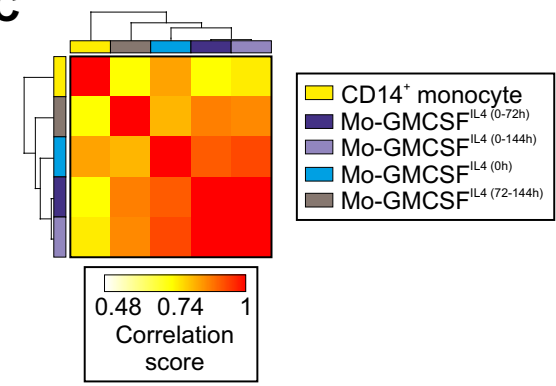

$\mathbf{F}$

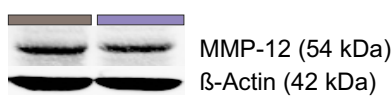

$\square$ Mo-GMCSF 1 (14 (72-144h) $\square$ Mo-GMCSF $^{1 \mathrm{L4}(0-144 \mathrm{~h})}$

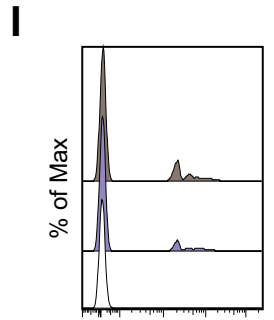

- YG beads -
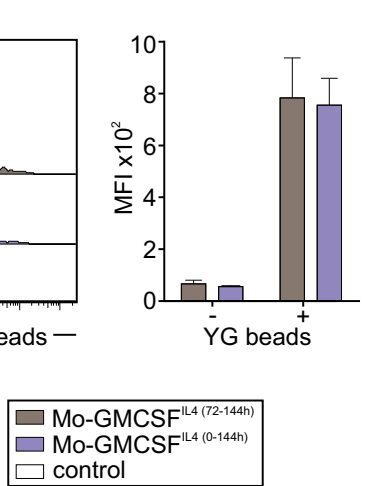

D

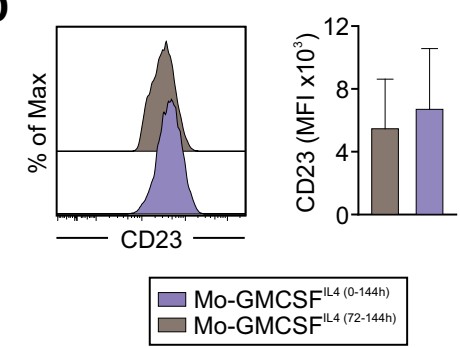

G

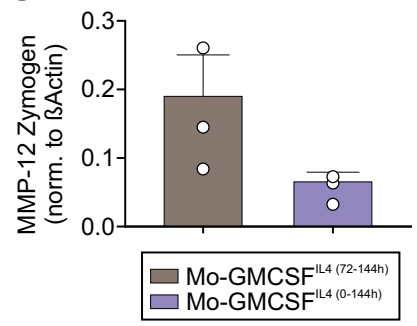

$J$

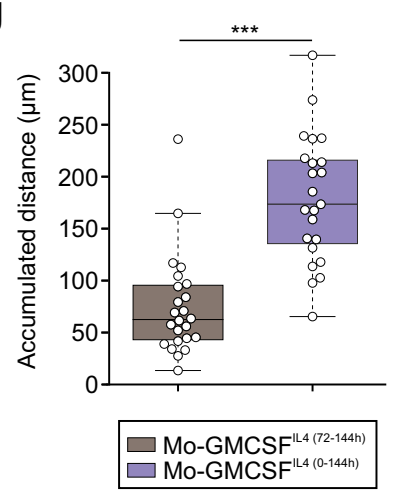

B

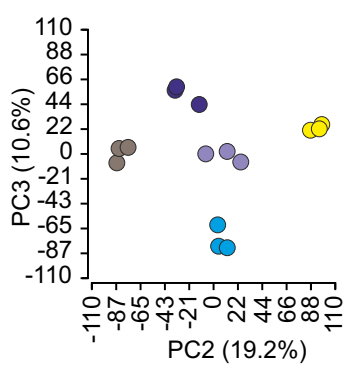

$\mathbf{E}$

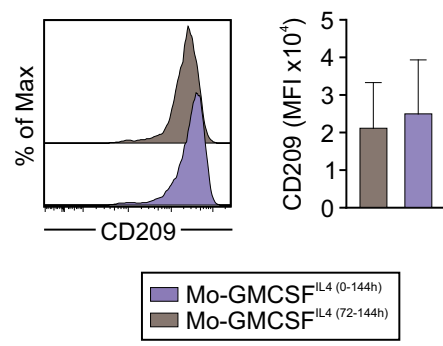

H

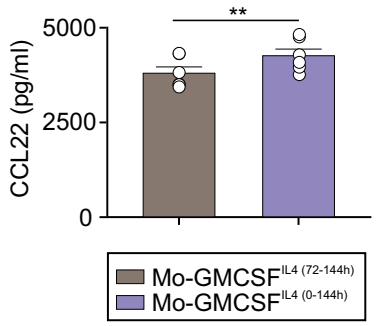

K

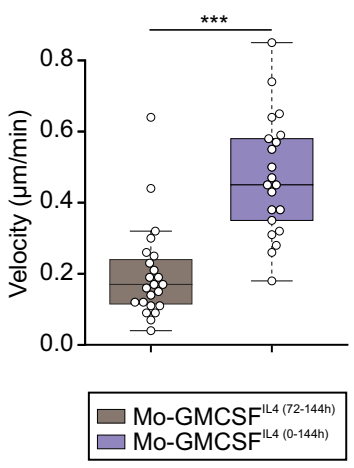


bioRxiv preprint doi: https://doi.org/10.1101/204180; this version posted October 16,2017 . The copyright holder for this preprint (which was not certified by peer review) is the author/funder. All rights reserved. No reuse allowed without permission.

\section{Supplementary figure 5}

A

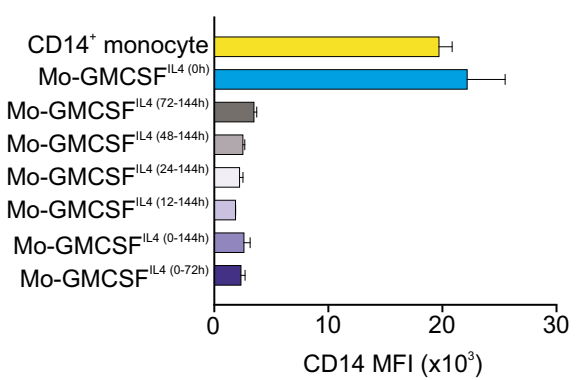

B

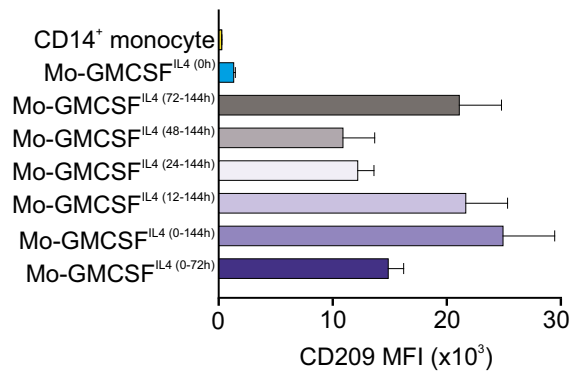

C

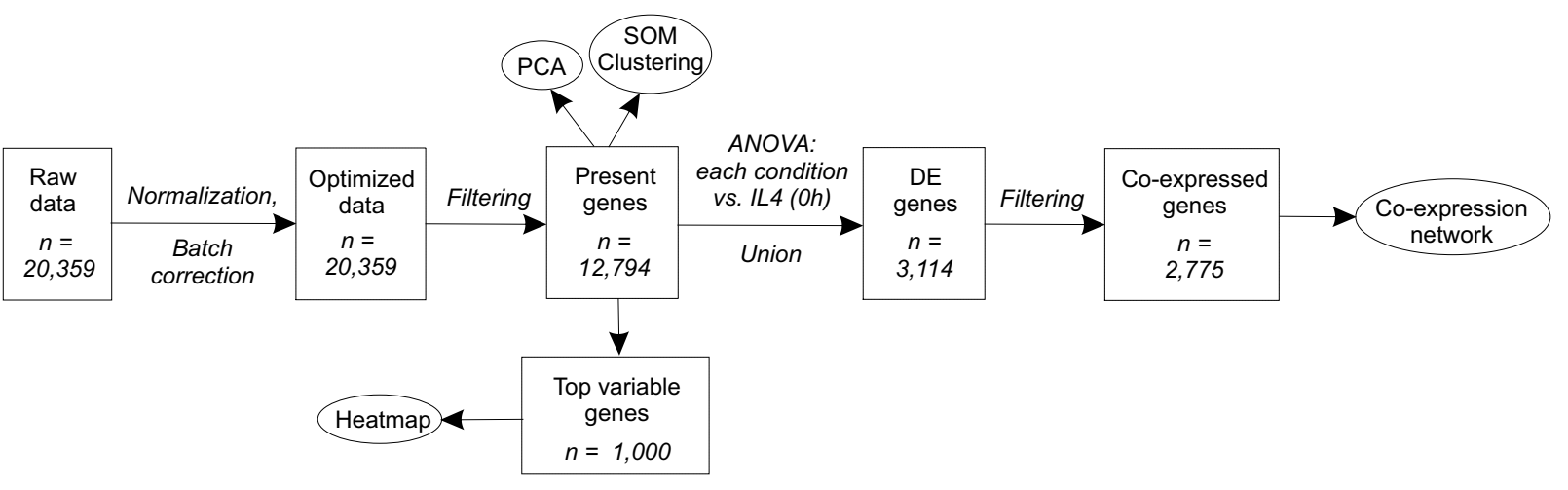


bioRxiv preprint doi: https://doi.org/10.1101/204180; this version posted October 16, 2017. The copyright holder for this preprint (which was not certified by peer review) is the author/funder. All rights reserved. No reuse allowed without permission.

\section{Supplementary figure 6}

A

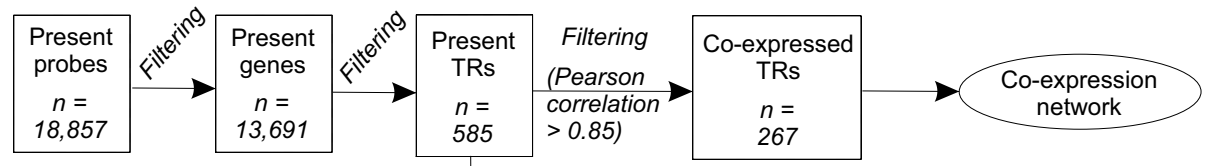

Fold-Change Mo-GMCSF $F^{\mathrm{LL}(0-72 h)}$ vs. CD14 ${ }^{+}$monocyte $>1.5$

\& Fold-Change Mo-GMCSF ${ }^{1 \mathrm{L4}(0-144 \mathrm{~h})}$ vs. CD14 ${ }^{+}$monocyte $>1.5$

\& Fold-Change Mo-GMCSF ${ }^{1 \mathrm{ll}(0-72 h)}$ vs. Mo-GMCSF $>1.5$

\& Fold-Change Mo-GMCSF ${ }^{1 / 4(0-144 h)}$ vs. Mo-GMCSF $>1.5$

$\&$ Fold-Change Mo-GMCSF vs. CD14 monocyte $>1$

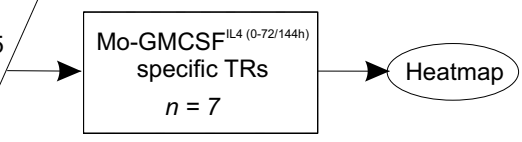

B

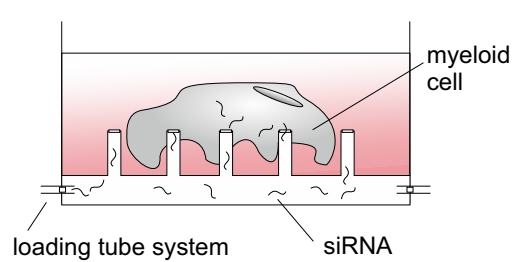

$\mathbf{F}$

G

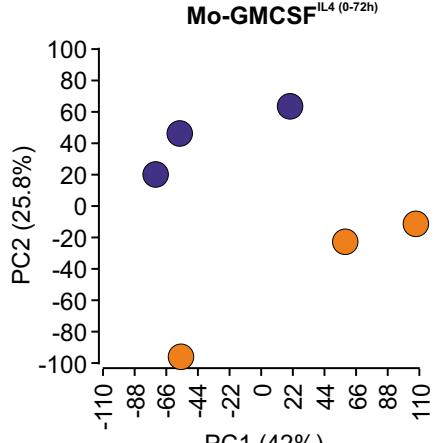

PC1 (42\%)

scrambled RNA $\square$ siRNA $\alpha$ NCOR2
C

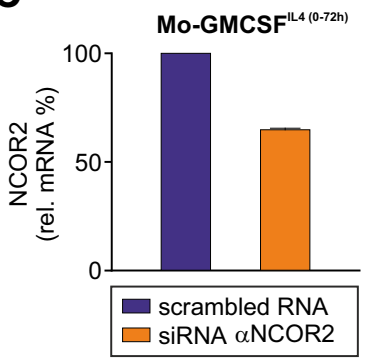

D

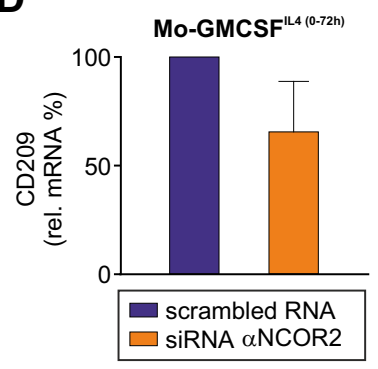

E

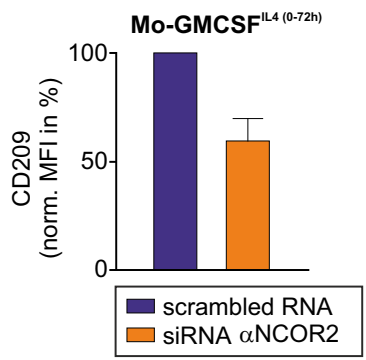

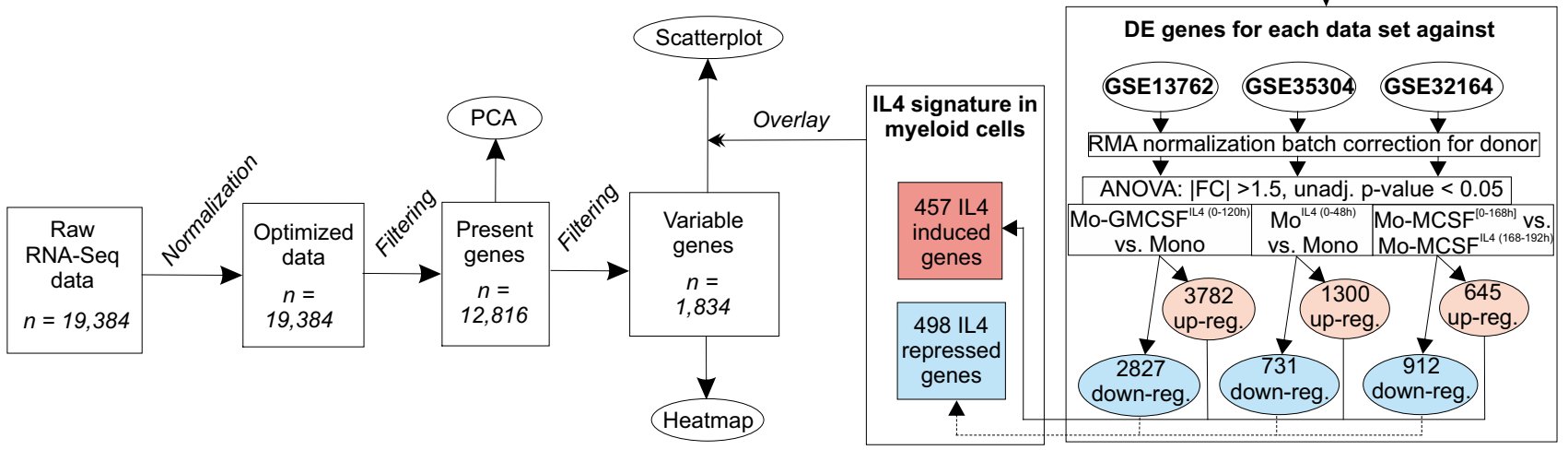

Usage of open available data sets of IL4 treated:

CD14 $^{+}$monocyte vs. Mo-GMCSF ${ }^{1 / 4(0-120 h)}$ (GSE13762) $\mathrm{CD}^{+} 4^{+}$monocyte vs Mo $\mathrm{Mo}^{1 \mathrm{~L}(0-48 \mathrm{~h})}$ (GSE35304) Mo-MCSF ${ }^{[0-168 \mathrm{~h}]}$ vs Mo-MCSF ${ }^{\mathrm{ll4}(168-192 \mathrm{~h})}$ (GSE32164) 